This article has been accepted for publication in MNRAS (c) 2018 The Authors. Published by Oxford University Press on behalf of the Royal Astronomical Society. All rights reserved. 


\title{
Observations of a nearby filament of galaxy clusters with the Sardinia Radio Telescope
}

\author{
V. Vacca, ${ }^{1 \star}$ M. Murgia, ${ }^{1}$ F. Govoni,${ }^{1}$ F. Loi,${ }^{1,2}$ F. Vazza,,${ }^{3,4,5}$ A. Finoguenov, ${ }^{6}$ \\ E. Carretti, ${ }^{1}$ L. Feretti, ${ }^{4}$ G. Giovannini,${ }^{3,4}$ R. Concu, ${ }^{1}$ A. Melis,${ }^{1}$ C. Gheller, ${ }^{7}$ \\ R. Paladino, ${ }^{4}$ S. Poppi, ${ }^{1}$ G. Valente, ${ }^{1,8}$ G. Bernardi, ${ }^{3,9,10}$ W. Boschin, ${ }^{11,12,13}$ \\ M. Brienza, ${ }^{14}$ T. E. Clarke, ${ }^{15}$ S. Colafrancesco, ${ }^{16}$ T. A. Enßlin, ${ }^{17}$ C. Ferrari, ${ }^{18}$ F. de \\ Gasperin, ${ }^{19}$ F. Gastaldello, ${ }^{20,21}$ M. Girardi, ${ }^{22,23}$ L. Gregorini, ${ }^{4}$ M. Johnston-Hollitt, ${ }^{24}$ \\ H. Junklewitz, ${ }^{25}$ E. Orrù, ${ }^{14}$ P. Parma, ${ }^{4}$ R. Perley ${ }^{26}$ and G. B. Taylor ${ }^{27}$ \\ Affiliations are listed at the end of the paper
}

Accepted 2018 April 22. Received 2018 January 4; in original form 2018 April 13

\begin{abstract}
We report the detection of diffuse radio emission which might be connected to a large-scale filament of the cosmic web covering a $8^{\circ} \times 8^{\circ}$ area in the sky, likely associated with a $z \approx 0.1$ overdensity traced by nine massive galaxy clusters. In this work, we present radio observations of this region taken with the Sardinia Radio Telescope. Two of the clusters in the field host a powerful radio halo sustained by violent ongoing mergers and provide direct proof of intracluster magnetic fields. In order to investigate the presence of large-scale diffuse radio synchrotron emission in and beyond the galaxy clusters in this complex system, we combined the data taken at $1.4 \mathrm{GHz}$ with the Sardinia Radio Telescope with higher resolution data taken with the NRAO VLA Sky Survey. We found 28 candidate new sources with a size larger and $\mathrm{X}$-ray emission fainter than known diffuse large-scale synchrotron cluster sources for a given radio power. This new population is potentially the tip of the iceberg of a class of diffuse large-scale synchrotron sources associated with the filaments of the cosmic web. In addition, we found in the field a candidate new giant radio galaxy.
\end{abstract}

Key words: acceleration of particles-magnetic fields-galaxies: clusters: intracluster medium-cosmology: observations - large-scale structure of Universe.

\section{INTRODUCTION}

One of the major challenges of the new generation of astronomical instruments is the detection of the magnetic cosmic web. According to cosmological simulations, half of the baryons expected in the Universe from the cosmic microwave background observations should be located in the filaments connecting galaxy clusters, in the form of a diffuse plasma (with median overdensities of about 10-30 and temperatures $10^{5}-10^{7} \mathrm{~K}$, see Cen \& Ostriker 1999; Davé et al. 2001). Owing to the low density and intermediate temperature range, the thermal component of the cosmic web is hardly detectable in $\mathrm{mm} / \mathrm{sub}-\mathrm{mm}$ through the Sunyaev-Zel'dovich (SZ) effect and in X-rays via bremsstrahlung emission. Thermal emission from plasma associated with filaments of the cosmic web has not yet been firmly detected. By using data from the Planck satellite of the region between the galaxy cluster couple A399-A401, the

\footnotetext{
*E-mail: valentina.vacca@inaf.it
}

Planck Collaboration VIII (2013) provided evidence of an SZ signal from the medium between the two clusters within their virial radii. Later, X-ray observations by Eckert et al. (2015) indicated structures coherent over scales of $8 \mathrm{Mpc}$, associated with the galaxy cluster A2744 and with the same spatial location of galaxy overdensities and dark matter. Recently, a signature of intergalactic filaments between close galaxy couples have been statistically reported using Planck data (de Graaff et al. 2017), while a statistical study based on a sample of 23 massive galaxy clusters conducted by Haines et al. (2017) showed that half of the expected cluster mass growth rate is due to X-ray groups in the infall region when clusters between the present epoch and $z \sim 0.2$ are considered.

A non-thermal component is expected to be present as well and to emit in the radio band via the synchrotron mechanism. This component is even more difficult to observe due to the expected very low density of cosmic rays and the weakness of the magnetic fields involved (e.g. Vazza et al. 2015). Magnetic fields in the large-scale structure of the Universe have been discovered only in the second 
half of the last century through the observation of large-scale diffuse synchrotron sources both at the centre and in the periphery of galaxy clusters, called respectively radio halos and relics (see e.g. Feretti et al. 2012). The properties of these sources in total intensity and polarization indicate magnetic fields, tangled on scales from a few $\mathrm{kpc}$ up to a few hundred kpc, characterized by central $\mu \mathrm{G}$ strengths that decline towards the periphery as a function of the thermal gas density of the intracluster medium (e.g. Murgia et al. 2004; Govoni et al. 2006, 2017; Vacca et al. 2010).

Numerical simulations suggest that cosmological filaments host weak magnetic fields (with strengths $\gtrsim \mathrm{nG}$, Brüggen et al. 2005; Ryu et al. 2008; Vazza et al. 2014), whose properties may reflect the primordial magnetic field strength and structure. Beyond a few Mpc from the cluster centre, only hints of the presence of non-thermal emission are available. Bridges of diffuse radio emission have been found to connect the galaxy cluster centre and periphery, as in the case of the Coma cluster (see Kronberg et al. 2007, and references therein), A2744 (Orrù et al. 2007), and A3667 (Carretti et al. 2013). Extended emission regions at a projected distance of $2 \mathrm{Mpc}$ from the cluster centre and probably connected with the large-scale structure formation processes have been detected in the galaxy cluster A2255 (Pizzo et al. 2008). At larger distances from the cluster centre, diffuse radio emission associated with the optical filament of galaxies $\mathrm{ZwCl} 2341.1+0000$ has been observed by Bagchi et al. (2002) and Giovannini et al. (2010), corresponding to a linear size larger than a few Mpc. Later, by using optical data, Boschin et al. (2013) found that this cluster belongs to a complex system undergoing a merger event, posing new questions on the nature of the observed diffuse emission that could be alternatively due to a two relics plus halo system. Diffuse emission along an optical filament has been recently detected also in the A3411-A3412 system (Giovannini et al. 2013) that could be powered by accretion shocks as material falls along the filament. According to an alternative explanation, the observed emission is evidence of re-acceleration of fossil electrons (van Weeren et al. 2013; Van Weeren et al. 2016). However, no clear and firm association of the filaments of the cosmic web with a radio signal has been found to date. The major limit to observation of the magnetization of large-scale structure is the scanty sampling of short $u v$-spacing in present interferometers: the Square Kilometre Array (SKA), and its precursors and path-finders can overcome this limit and have the capabilities to detect radio emission from the cosmic web at frequencies below $1.4 \mathrm{GHz}$, if the magnetization of the medium is at least about 1 per cent of the energy in the thermal gas (Vazza et al. 2015).

Waiting for the SKA, a valuable contribution to the study of the magnetization of the cosmic web through its synchrotron radio emission can be given by the analysis of radio emission with simultaneously interferometric and single-dish telescopes. By combining single-dish data collected with the $305 \mathrm{~m}$ Arecibo telescope with interferometric data collected with the Dominion Radio Astrophysical Observatory at $0.4 \mathrm{GHz}$, Kronberg et al. (2007) imaged a region of the sky of $8^{\circ}$ around the Coma cluster. They detected large-scale synchrotron emission in the direction of the cluster, corresponding to a linear size of about $4 \mathrm{Mpc}$ if located at the same redshift as Coma. A possible intercluster source associated with the filament between A2061 and A2067 has been claimed by Farnsworth et al. (2013), after subtracting point sources from $100 \mathrm{~m}$ Green Bank Telescope (GBT) observations at $1.4 \mathrm{GHz}$ by using interferometric data. Extragalactic sources are typically observed with interferometers to reach a suitable spatial resolution. Nevertheless, full-synthesis interferometric observations do not recover information on angular scales larger than those corre- sponding to their minimum baseline and do not have sensitivity on such large scales. On the contrary, single-dish telescopes reveal emission over scales as large as the size of the scanned region. These characteristics make single-dishes and interferometers complementary instrument for the study of large-scale synchrotron sources. The ability to reconstruct emission from large-scale structures of single-dishes combined with the high-spatial resolution of interferometers, allows us to recover the emission of sources covering angular size larger than a few tens of arcminutes and to separate this emission from that of possible embedded compact sources.

Great effort in the last years has been devoted to constrain the magnetic field strength beyond the cluster volume both on the basis of radio observations alone and with multiwavelength data. Brown et al. (2017), constrained the magnetic field in the cosmic web to be in the range $0.03-0.13 \mu \mathrm{G}(3 \sigma)$ and the primordial magnetic field less than $1 \mathrm{nG}$ by cross-correlating the radio emission observed with the S-band Polarization All Sky Survey radio survey at $2.3 \mathrm{GHz}$ with a simulated model of the local synchrotron cosmic web. By cross-correlating radio observations at $180 \mathrm{MHz}$ obtained with the Murchison Widefield Array and galaxy number density estimates from the Two Micron All-Sky Survey and the Wide-field Infrared Explorer redshift catalogues, Vernstrom et al. (2017) put limits on the magnetic field strength of the cosmic web of $0.03-1.98 \mu \mathrm{G}$ $(1 \sigma)$.

In this paper, we present a study of the radio emission of a new filament of the cosmic web by using new single-dish observations and interferometric data. The paper is organized as follows. In Section 2, a description of the system is given. In Section 3, we summarize the radio observations. In Section 4, we present the combination of single data with interferometric snapshot observations. In Sections 5 and 6, we report the X-ray and sub-millimetre properties of the sources in this region. In Sections 7 and 8, we present and discuss our results and in Section 9 we present our conclusions. In Appendices A and B, we check radio properties of a sample of compact sources and of interesting radio galaxies in the field.

In the following, we consider a flat Universe and adopt a $\Lambda$ cold dark matter $(\Lambda \mathrm{CDM})$ cosmology with $H_{0}=67.3 \mathrm{~km} \mathrm{~s}^{-1} \mathrm{Mpc}^{-1}$, $\Omega_{\mathrm{m}}=0.315$, and $\Omega_{\Lambda}=0.685$ (Planck Collaboration XVI 2014).

\section{THE FILAMENTS OF THE COSMIC WEB}

Clusters form at the intersection of filaments of the cosmic web through which they accrete material during the processes that cause their formation. We identified a rich region in the radio sky that covers a size of $8^{\circ} \times 8^{\circ}$ and hosts 43 clusters. Among these, 13 have either a spectroscopic or photometric redshift identification (see Table 1). These redshifts range from 0.015 to 0.425 as shown in the histogram in Fig. 1, which clearly shows a predominant peak around $z=0.11$ with standard deviation 0.02 . This peak is caused by nine clusters with redshift in the range $0.08 \lesssim z \lesssim$ 0.15, i.e. A523, A525, RXC J0503.1+0607, A529, A515, A526, RXC J0506.9+0257, A508, and A509. Two clusters have a redshift smaller than 0.08: ZwCl 0510.0+0458 A and ZwCl 0510.0+0458 B, located along the same line of sight, at RA J2000 05:12:39.4 and Dec. J2000 +05:01:31, at a distance of $8 \mathrm{Mpc}$. Two clusters have redshift larger than 0.12: A520, and MACS J0455.2+0657. MACS J0455.2+0657 is the most distant system in the field. It is a very luminous, massive, and distant galaxy cluster, part of the MAssive Cluster Survey (MACS), see Mann \& Ebeling (2012). According to these authors, this is a pronounced cool core, char- 
Table 1. Clusters in the field with known redshift.

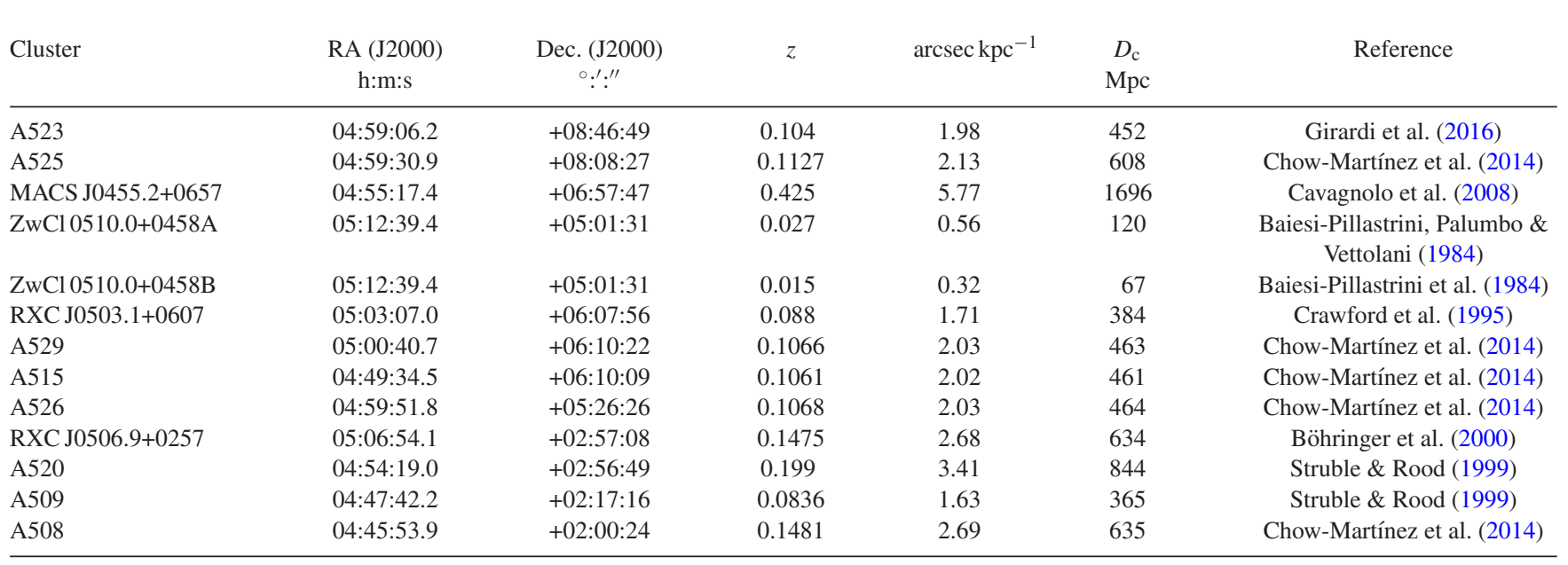

Col 1: Cluster name; Col 2 and 3: cluster coordinates; Col 4: redshift; Col 5: linear scale conversion factor; Col 6: comoving radial distance; Col 7: reference for the redshift.

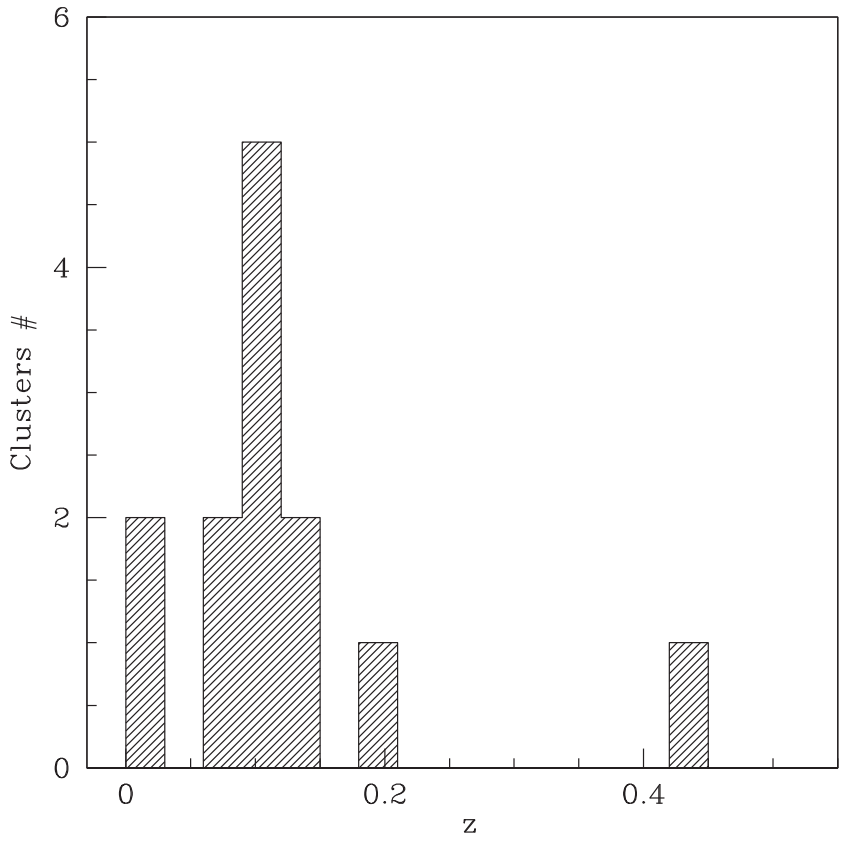

Figure 1. Redshift distribution of clusters in the field of view with a redshift identification.

acterized by a single brightest cluster galaxy (BCG) and by a perfect alignment between the X-ray peak and the BCG. In addition, we can identify in the field 29 additional clusters belonging to the Zwicky Clusters of Galaxies catalogue (Zwicky, Karpowicz \& Kowal 1965) and one belonging to the Hubble Space Telescope Medium Deep Survey Cluster Sample (Ostrander et al. 1998) but, no redshift information is available in the literature for them. The full list of these clusters along with their coordinates is given in Table 2.

Some among the clusters in the field have been already found to trace the cosmic web structure. Einasto et al. (2001) identify the superclusters SCL 061 and SCL 062 that include respectively A509-A526 and A515-A523-A525-A529-A532 (A532 is outside our field of view). Later, a new classification has been performed by Chow-Martínez et al. (2014), who identify the superclus-
Table 2. Clusters in the field without redshift information.

\begin{tabular}{|c|c|c|}
\hline Cluster & $\begin{array}{l}\text { RA (J2000) } \\
\text { h:m:s }\end{array}$ & $\begin{array}{c}\text { Dec. }(\mathrm{J} 2000) \\
\circ:^{\prime \prime}{ }^{\prime \prime}\end{array}$ \\
\hline $\mathrm{ZwCl} 0440.1+0514$ & $04: 42: 45.5$ & $+05: 19: 37$ \\
\hline $\mathrm{ZwCl} 0440.3+0547$ & $04: 42: 58.2$ & $+05: 52: 36$ \\
\hline $\mathrm{ZwCl} 0440.9+0407$ & $04: 43: 32.3$ & $+04: 12: 34$ \\
\hline $\mathrm{ZwCl} 0441.7+0423$ & $04: 44: 20.6$ & $+04: 28: 30$ \\
\hline $\mathrm{ZwCl} 0444.7+0828$ & $04: 47: 25.2$ & $+08: 33: 18$ \\
\hline $\mathrm{ZwCl} 0445.1+0223$ & $04: 47: 42.4$ & $+02: 28: 16$ \\
\hline $\mathrm{ZwCl} 0445.6+0539$ & $04: 48: 16.1$ & $+05: 44: 14$ \\
\hline $\mathrm{ZwCl} 0446.2+0235$ & $04: 48: 48.6$ & $+02: 40: 12$ \\
\hline $\mathrm{ZwCl} 0446.6+0150$ & $04: 49: 11.8$ & $+01: 55: 10$ \\
\hline $\mathrm{ZwCl} 0448.2+0919$ & $04: 50: 56.2$ & $+09: 24: 03$ \\
\hline $\mathrm{ZwCl} 0448.5+0551$ & $04: 51: 10.3$ & $+05: 56: 02$ \\
\hline $\mathrm{ZwCl} 0451.3+0159$ & $04: 53: 54.0$ & $+02: 03: 51$ \\
\hline $\mathrm{ZwCl} 0452.1+0627$ & $04: 54: 47.0$ & $+06: 31: 47$ \\
\hline HSTJ $045648+03529$ & $04: 56: 48.3$ & $+03: 52: 57$ \\
\hline ZwCl 0454.3+0534 & $04: 56: 58.0$ & $+05: 38: 38$ \\
\hline $\mathrm{ZwCl} 0455.3+0155$ & $04: 57: 53.9$ & $+01: 59: 34$ \\
\hline $\mathrm{ZwCl} 0455.2+0746$ & $04: 57: 54.5$ & $+07: 50: 34$ \\
\hline $\mathrm{ZwCl} 0457.0+0511$ & $04: 59: 39.6$ & $+05: 15: 26$ \\
\hline $\mathrm{ZwCl} 0458.2+0137$ & 05:00:47.6 & $+01: 41: 22$ \\
\hline $\mathrm{ZwCl} 0458.5+0102$ & 05:01:04.9 & $+01: 06: 20$ \\
\hline ZwCl 0458.5+0536 & 05:01:10.1 & $+05: 40: 20$ \\
\hline $\mathrm{ZwCl} 0459.2+0212$ & $05: 01: 48.2$ & $+02: 16: 17$ \\
\hline ZwCl 0459.6+0606 & $05: 02: 16.7$ & $+06: 10: 15$ \\
\hline $\mathrm{ZwCl} 0459.8+0943$ & $05: 02: 32.8$ & $+09: 47: 14$ \\
\hline $\mathrm{ZwCl} 0502.0+0350$ & 05:04:38.1 & $+03: 54: 05$ \\
\hline $\mathrm{ZwCl} 0502.1+0201$ & $05: 04: 42.0$ & $+02: 05: 05$ \\
\hline $\mathrm{ZwCl} 0503.1+0751$ & $05: 05: 48.7$ & $+07: 55: 01$ \\
\hline $\mathrm{ZwCl} 0504.9+0417$ & 05:07:32.6 & $+04: 20: 53$ \\
\hline $\mathrm{ZwCl} 0505.1+0655$ & $05: 07: 47.6$ & $+06: 58: 52$ \\
\hline $\mathrm{ZwCl} 0508.8+0241$ & $05: 11: 24.8$ & $+02: 44: 36$ \\
\hline
\end{tabular}

Col 1: Cluster name; Col 2 and 3: cluster coordinates.

ter MSCC 145 consisting of the clusters A515-A526-A525-A529. These systems are poorly known in radio, optical, and X-rays, with the exception of A523 and A520, which are the only two systems in the field known to host diffuse large-scale synchrotron emission (see Sections 2.1 and 2.2). 
Table 3. Data.

\begin{tabular}{lccccc}
\hline Date & Receiver & Target & FOV & OFT scan axis & Calibrators \\
\hline 2016 July 14 & L-band & A520-A526-A523 & $8^{\circ} \times 8^{\circ}$ & $1 \times($ RA+Dec. $)$ & Time on source \\
2016 July 23 & L-band & A520-A526-A523 & $8^{\circ} \times 8^{\circ}$ & $1 \times($ RA+Dec. $)$ & $3 \mathrm{C} 286,3 \mathrm{C} 147,3 \mathrm{C} 138$ \\
2016 July 22 & -band & A520 138 & $10 \mathrm{~h}$ \\
2016 July 22 & $L$-band & A523 & $3^{\circ} \times 3^{\circ}$ & $1 \times(\mathrm{RA}+$ Dec. $)$ & 3C $84,3 \mathrm{C} 147,3 \mathrm{C} 138$ \\
2016 July 22 & L-band & A526 & $3^{\circ} \times 3^{\circ}$ & $1 \times(\mathrm{RA}+$ Dec. $)$ & 3C $84,3 \mathrm{C} 147,3 \mathrm{C} 138$ \\
\end{tabular}

\subsection{Abell 520}

This galaxy cluster is a dynamically young system, undergoing a strong merger event in the NE-SW direction (Govoni et al. 2004). In radio, it is famous for hosting an extended synchrotron source with a largest linear size (LLS) of $1.4 \mathrm{Mpc}$, originally classified as a radio halo (Giovannini, Tordi \& Feretti 1999; Govoni et al. 2001), with the south-west edge of the radio emission coincident with a bow shock, as found by Markevitch et al. (2005). A recent re-analysis of the radio emission in the cluster revealed that the radio halo has a flux density at $1.4 \mathrm{GHz}$ of $S_{1.4 \mathrm{GHz}}=(16.7 \pm 0.6) \mathrm{mJy}$ (Vacca et al. 2014). The peculiar morphology and properties of the source do not reflect the typical properties of radio halos and raise new questions about its nature, as noted already by Govoni et al. (2001). By using optical observations from the Telescopio Nazionale Galileo and the Isaac Newton Telescope facilities, Girardi et al. (2008) found evidence that the cluster formation is taking place at the crossing of three filaments: one in the north-east/south-west direction, one in the east-west direction, and one almost aligned with the line of sight.

\subsection{Abell 523}

According to Chow-Martínez et al. (2014), A523 is an isolated galaxy cluster. It consists of an irregular south/south-west subcluster strongly interacting with a more compact sub-cluster on the north/north-east direction (Girardi et al. 2016). The system has been discovered to host large-scale diffuse radio emission (Giovannini et al. 2011) in the form of a radio halo located at the centre of the cluster with a flux $S_{1.4 \mathrm{GHz}}=(59 \pm 5) \mathrm{mJy}$ and a largest linear angular size of $12 \mathrm{arcmin}$. According to a re-analysis of the radio data by Girardi et al. (2016), this source has a flux density at $1.4 \mathrm{GHz}$ of $S_{1.4 \mathrm{GHz}}=(72 \pm 3) \mathrm{mJy}$, an LLS of $1.3 \mathrm{Mpc}$ and it is characterized by a polarized signal. Polarization is uncommon among radio halos. It has been detected in only other two cases, A2255 (Govoni et al. 2005) and MACS J0717+3745 (Bonafede et al. 2009), and indicates an intracluster magnetic field fluctuating on scales as large as hundreds of kpc. Compared to the other radio halos, this system is underluminous in X-rays with respect to radio, opening new questions on the formation of radio halos and their link with merger events.

\section{RADIO OBSERVATIONS}

In order to look for the presence of diffuse emission from lowdensity environments connecting galaxy clusters, we observed with the SRT a region of the sky hosting nine clusters with redshift $z \approx 0.1$ : A523, A525, RXC J0503.1+0607, A529, A515, A526, RXC J0506.9+0257, A509, and A508, see Table 1. The radio observations presented in this paper were done in the context of the SRT Multi-frequency Observations of Galaxy Clusters program (SMOG, PI. Matteo Murgia; see Govoni et al. 2017; Loi et al. 2017, for a description of the project). We observed an area of $8^{\circ} \times 8^{\circ}$ centred at right ascension (RA) 05h:00m:00.0s and declination (Dec.) $+05^{\circ}: 48^{\prime}: 00.0^{\prime \prime}$, for a total exposure time of about $18 \mathrm{~h}$ and three smaller fields of view centred respectively on the galaxy clusters A520, A526, and A523 over a region of $3^{\circ} \times 3^{\circ}$ of about $1.5 \mathrm{~h}$ each. The observations were taken with the $L$ - and $P$-band dualfrequency coaxial receiver for primary-focus operations (Valente et al. 2010). We used the $L$-band component only covering the frequency range $1.3-1.8 \mathrm{GHz}$. The data stream was recorded with the SArdinia Roach2-based Digital Architecture for Radio Astronomy (SARDARA; Melis et al. 2018) back-end. The configuration used had $1500 \mathrm{MHz}$ of bandwidth and 16384 channels $\sim 90 \mathrm{kHz}$ each, full-Stokes parameters, and sampling at 10 spectra per second. We opted for the on-the-fly mapping strategy scanning the sky alternatively along the RA and Dec. direction with a telescope scanning speed of 6 arcmin s ${ }^{-1}$ and an angular separation of 3 arcmin between scans, this choice guarantees a proper sampling of the SRT beam whose full width at half-maximum (FWHM) is 13.9 arcmin $\times 12.4$ arcmin at $1550 \mathrm{MHz}$, the central frequency of these observations. Since the sampling time is $100 \mathrm{~ms}$, the individual samples are separated in the sky by 36 arcsec along the scanning direction. A detailed summary of the SRT observations is given in Table 3.

The calibration and the imaging of the data were performed with the proprietary Single-dish Spectral-polarimetry Software (scuBE; Murgia et al. 2016). We used 3C 147 as band-pass and flux density calibrator and the flux density scale of Perley \& Butler (2013) was assumed. We flagged about 23 percent of the data because of radio frequency interference. After calibration, a baseline was fitted to the data in order to subtract any emission not related to the target (due e.g. to our Galaxy). The subtraction of the baseline has been performed as follows. First, we convolved the NVSS image of this region to the resolution of the SRT. We used this image to identify empty regions in the SRT image. The linear fit of the emission of these regions was then subtracted from the data. The baseline-subtracted data were then projected in a regular 3D (RA-Dec.-observing frequency) grid with a spatial resolution of 3 arcmin pixel ${ }^{-1}$ in order to guarantee a beam FWHM sampling of four pixels. These steps were performed in each frequency channel. The resulting frequency cube was stacked and de-stripped by mixing the Stationary Wavelet Transform coefficients (see Murgia et al. 2016, for details). This image was then used to produce a new mask and the procedure was repeated. This procedure was performed for each data set separately and the images produced for each data set were averaged.

Finally, we applied a hard threshold denoising algorithm on scales below two pixels ( 1 pixel $=3$ arcmin) as the signal amplitude is expected to be negligible with respect to the noise amplitude on these angular scales. The denoised image obtained by the combination of all the SRT data is shown in Fig. 2 in colours and contours. The noise level $1 \sigma$ of the radio image is $20 \mathrm{mJy}_{\text {beam }}{ }^{-1}$, comparable with the confusion noise for this frequency band at the SRT resolu- 


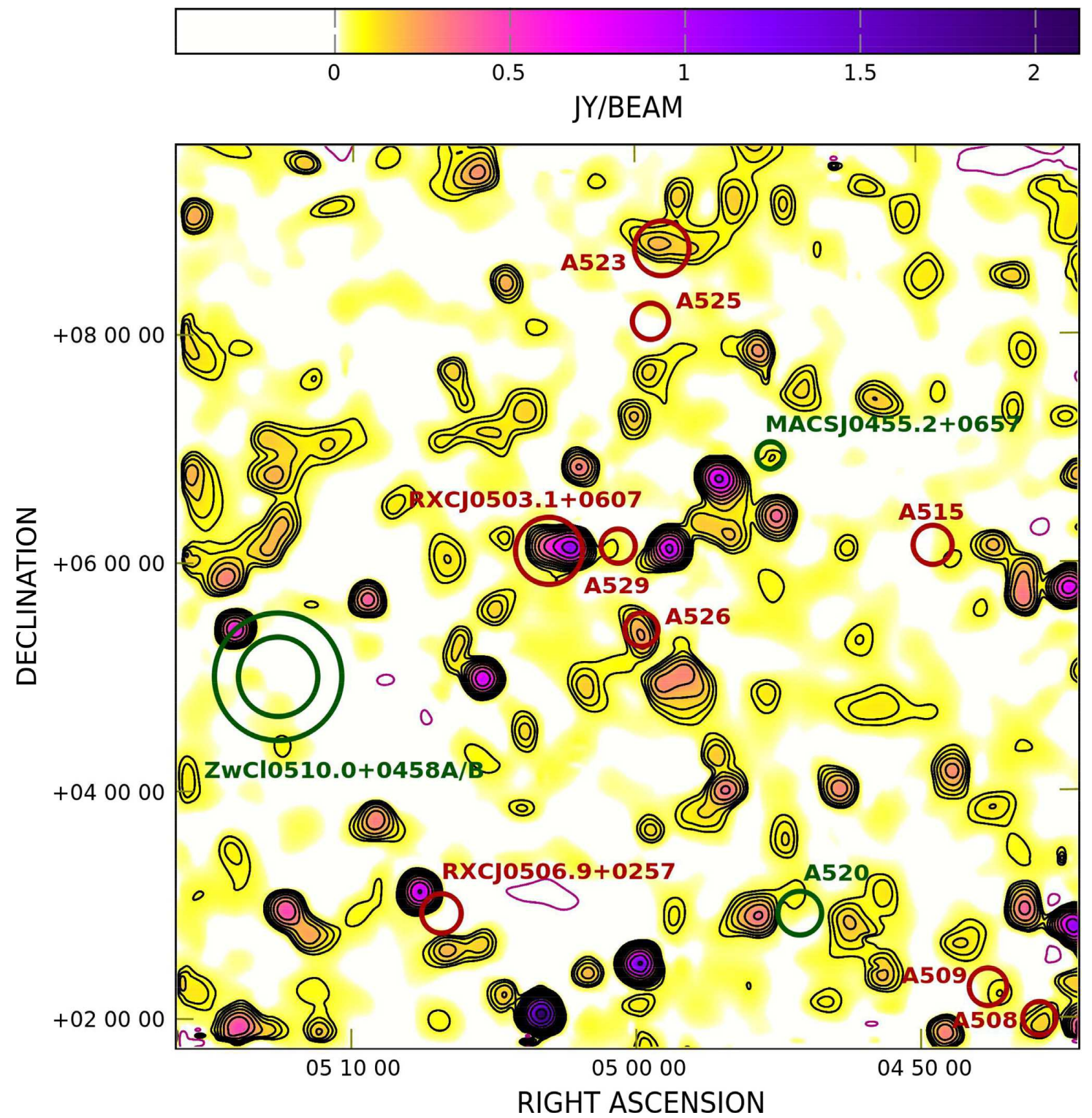

Figure 2. SRT image in colours and contours in the frequency range $1.3-1.8 \mathrm{GHz}$. The contours are $-3 \sigma, 3 \sigma\left(\sigma=20 \mathrm{mJy}^{\mathrm{beam}}{ }^{-1}\right)$ and the remaining scale by a factor $\sqrt{2}$. Negative contours are represented in magenta, while positive contours are in black. The angular resolution of the images is 13.9 arcmin $\times 12.4$ arcmin and $\mathrm{BPA}=-58.5^{\circ}$ at the centre of the observing band. The circles and labels identify the position of the galaxy clusters in the field of view with known redshift. The size of the circles is proportional to $R_{200}$ of the corresponding clusters (see Table 4), large circles are close-by clusters while small circles are distance clusters. For systems with an upper limit for $R_{200}$, we used the value of the upper limit. We highlight in red clusters with redshift between 0.08 and 0.15 , in green clusters with redshift outside this range.

tion. The zero-level of the image is $\sim 25$ per cent of the noise level. A detailed description of the calibration and imaging procedures of the SRT data are given in Murgia et al. (2016) and Govoni et al. (2017).

The circles and labels in Fig. 2 indicate the position in the field of the clusters with a redshift identification. This figure reveals that most of the nine clusters with redshift $\approx 0.1$ are all located along a line crossing the field of view from north to south and connecting the galaxy clusters A523 and A508, passing through A525, RXC J0503.1+0607, A529, A526, and A509, while RXC J0506.9+0257 and A515 are respectively slightly southeast and slightly west of the filament. A520 has a redshift higher than the average redshift of this group. A possible explanation is that A520 appears to belong to this region of the sky only be- cause of projection effects. Alternatively, the filament could be partially located in the plane of the sky and partially oriented along the line of sight in the direction of A520. This is supported by the findings of Girardi et al. (2008) which suggest the hypothesis that A520 could be connected with the filament at $z \approx 0.1$ examined in this work. However, as shown in Table 1 , the redshift of A520 implies a distance along the line of sight of about $410 \mathrm{Mpc}$ therefore the hypothesis of a connection is still controversial.

\subsection{SRT beam and calibration scale}

We cross-checked the calibration procedures by comparing the SRT flux scale with the NVSS flux scale. To perform the com- 

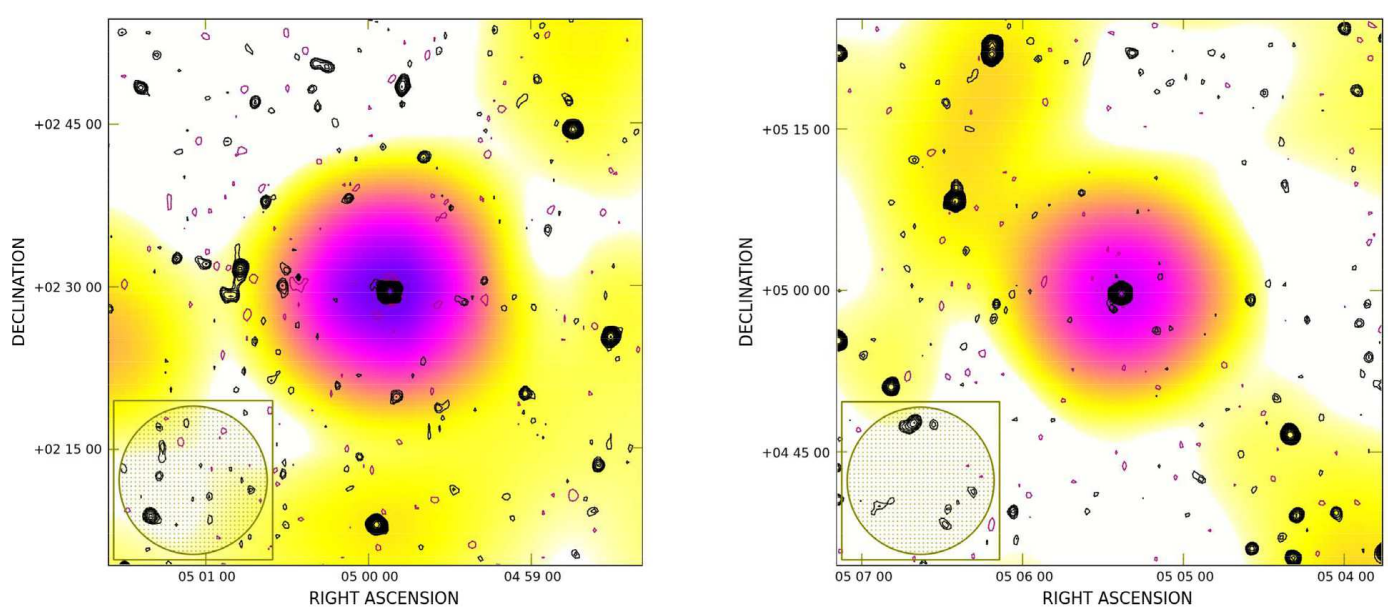

Figure 3. Sources used for the beam measurement (left-hand panel) and for the comparison of the NVSS and SRT flux density scales (right-hand panel). The SRT image in the same frequency range as the NVSS is shown in colours, while the NVSS emission is shown in contours. The contour levels are -1.35 , $1.35 \mathrm{mJy}$ beam $^{-1}$ (with a beam of 45 arcsec) and increase by a factor $\sqrt{2}$, positive contours shown in black and negative contours shown in magenta. The beam in the bottom left corner represents the SRT spatial resolution of 13.66 arcmin as found in Section 3.1. The colorbar is the same as Fig. 2.
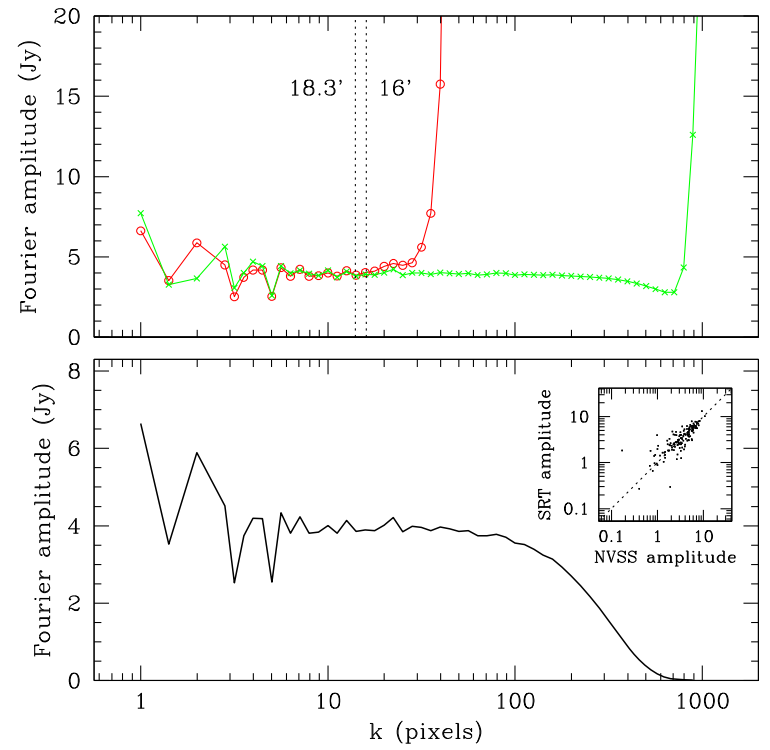

Figure 4. Top panel: Power spectrum of the SRT data (red) and power spectrum of the NVSS data (green). The dashed vertical black lines identify the overlapping region used to adjust the flux density scales. Bottom panel: Tapered combined power spectrum. In the inset, the comparison between the amplitude of the SRT and of the NVSS data in the region of overlapping (18.3-16 arcmin) in the Fourier space is shown.

parison, we produced SRT images in the same frequency range as the NVSS images, we tailored from the whole SRT bandwidth $(1.3-1.8 \mathrm{GHz})$ two smaller sub-bands centred at the same frequencies and with the same width of the NVSS observations (1364.9$1414.9 \mathrm{MHz}$ and $1435.1-1485.1 \mathrm{MHz}$ ) and then binned them in one image.

As a first step, we measured the beam of the SRT images by fitting the emission of the brightest isolated point source in the field with the software SCUBE (Murgia et al. 2016). This compact source is a quasar with redshift $z=2.384$ (Xu \& Han 2014) and is located at RA J2000 04h:59m:53.94s and Dec. J2000 02 :29':39.72" (see Fig. 3, left-hand panel). By fitting the peak emission with a circular
2D Gaussian with four free parameters (peak, $x$ and $y$ coordinates of the centre, and FWHM), we obtained for the SRT beam an FWHM of $\theta=(820 \pm 1)$ arcsec.

We then compared the SRT and NVSS flux scales by fitting the peak brightness of the brightest non-variable isolated point source. The source is located at RA J2000 05h:05m:24.21s and Dec. J2000 $04^{\circ}: 59^{\prime}: 48.44^{\prime \prime}$ (see Fig. 3, right-hand panel). During the fitting procedure the beam FWHM was kept fixed to the value derived above. The position of the peak as measured by the SRT and by the NVSS agrees within 15 arcsec. The peak in the two images are in agreement within about 10 percent, with a value respectively of $(0.96 \pm 0.03)$ and $(0.86 \pm 0.03) \mathrm{Jy}$ beam $^{-1}$. Note that the source used for the fit is not the brightest source in the field but one among the brightest isolated sources. Indeed, we prefer to discard non-isolated sources because of possible blending of sources. Moreover, we note that the flux scale of the NVSS and of SRT observations are based respectively on the scales by Baars et al. (1977) and Perley \& Butler (2013), and they agree within 1 per cent.

\section{COMBINATION OF SINGLE-DISH AND INTERFEROMETRIC DATA}

In this section, we present the combination of our single-dish data with mosaic interferometric observations taken from the NVSS, following the approach described by Loi et al. (2017). Single-dish observations can recover a maximum angular scale corresponding to the angular size of the scanned region but they miss high-spatial resolution. Interferometers can reach high-spatial resolutions but are limited by the maximum angular scale corresponding to their minimum baseline. In the case of a full-synthesis Very Large Array (VLA) observation this translates to $\sim 16$ arcmin for the most compact configuration at $L$ band. For snapshot observations as the NRAO VLA Sky Survey (NVSS; Condon et al. 1998) this number should be divided by two ${ }^{1}$ but we consider the full angular size since the NVSS image we used has been obtained with a mosaic.

${ }^{1}$ https://science.nrao.edu/facilities/vla/docs/manuals/oss/performance/res olution 

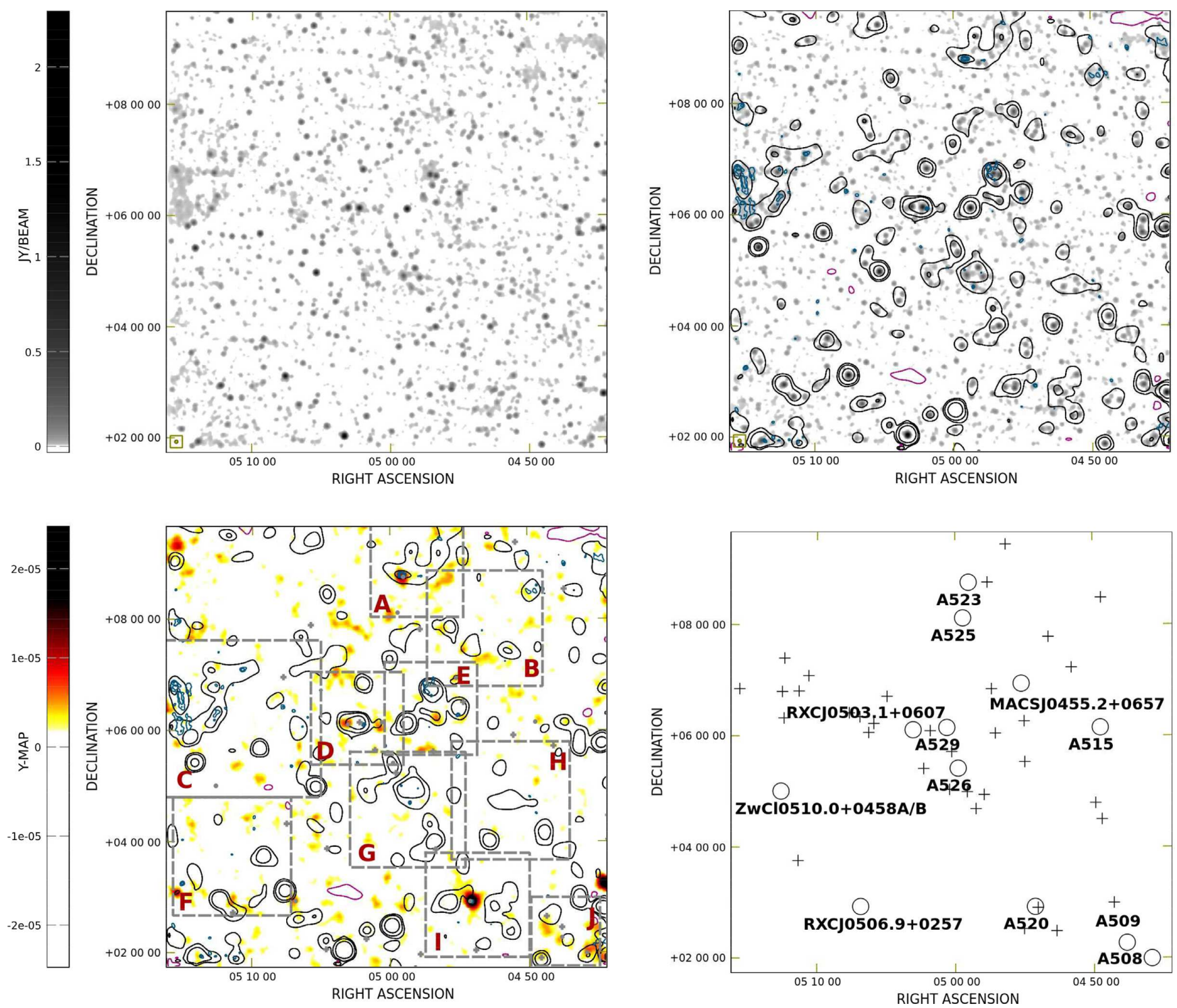

Figure 5. Top left panel: SRT+NVSS image in colours (FWHM 3.5 arcmin). Top right panel: Contours of the SRT image $\left(-60,60,150,500 \mathrm{mJy}^{\mathrm{beam}}{ }^{-1}\right.$, negative in magenta and positive in black; resolution $13.9 \operatorname{arcmin} \times 12.4 \mathrm{arcmin}$ ) and of the SRT+NVSS image after compact-source subtraction (starting at $3 \sigma$ and increasing by a factor $\sqrt{2}$, in blue; $\sigma=2.5 \mathrm{mJy}_{\text {beam }}{ }^{-1}$, resolution $3.5 \operatorname{arcmin} \times 3.5 \operatorname{arcmin}$ ) overlaid on the SRT+NVSS image in colours (FWHM $3.5 \mathrm{arcmin})$. Bottom left panel: SRT and SRT+NVSS after point-source subtraction in contours overlaid on the Planck Y-map (see the text) in colours. The contours from SRT and from the residual SRT+NVSS image are the same as in the top right panel. The resolution of the Planck Y-map is 10 arcmin. Dots indicate the position of clusters with redshift identification (see Table 1), while crosses indicate clusters without redshift identification (see Table 2). Bottom right panel: Location of clusters with known redshift (circles and labels) and of the sources discussed in this paper (crosses), see Table 5.

By combining the SRT and NVSS data, we retain the ability to observe large-scale structures up to $8^{\circ}$ and, at the same time, the resolution of the VLA in D configuration at $1.4 \mathrm{GHz}$, i.e. 45 arcsec. The combination has been performed in the Fourier domain with the sCUBE software package (Murgia et al. 2016). The SRT has a size of $64 \mathrm{~m}$, while the VLA in its most compact configuration has a minimum baseline of $35 \mathrm{~m}$, this means that there is a common range of spacing and consequently of wavenumbers $k$ in the Fourier space. ${ }^{2}$ We combined the NVSS image with the SRT image obtained in the

${ }^{2}$ For practical purposes, in our algorithm we define the wavenumber $k=$ $0.5 \frac{\Delta \times N_{\mathrm{pix}}}{\theta_{\mathrm{A}}}$, where $\Delta=15 \mathrm{arcsec}_{\mathrm{pixel}}{ }^{-1}$ is the angular size of the pixel of the image, $N_{\text {pix }}=2048$ is the number of pixels in the image along one side, and $\theta_{\mathrm{A}}$ is the angular scale in arcsec. same frequency range and select in this image the same region of the sky as the NVSS image, so that they should contain the same radio power.

In order to combine the two images we required that the two power spectra have the same power spectral density in the inner portion of the overlapping region in Fourier space, corresponding to angular scales between 16 and 18.3 arcmin. This requirement translates into a scaling factor of the SRT power spectrum normalization of $\approx 1.003$. In Section 3.1, by comparing the flux of a single source in the two images, we found that the two flux density scales agree within 10 per cent. In this section we make a more robust statistical comparison, by using all the sources available in the field. This agreement confirms the accuracy of the SRT flux density scale calibration. 


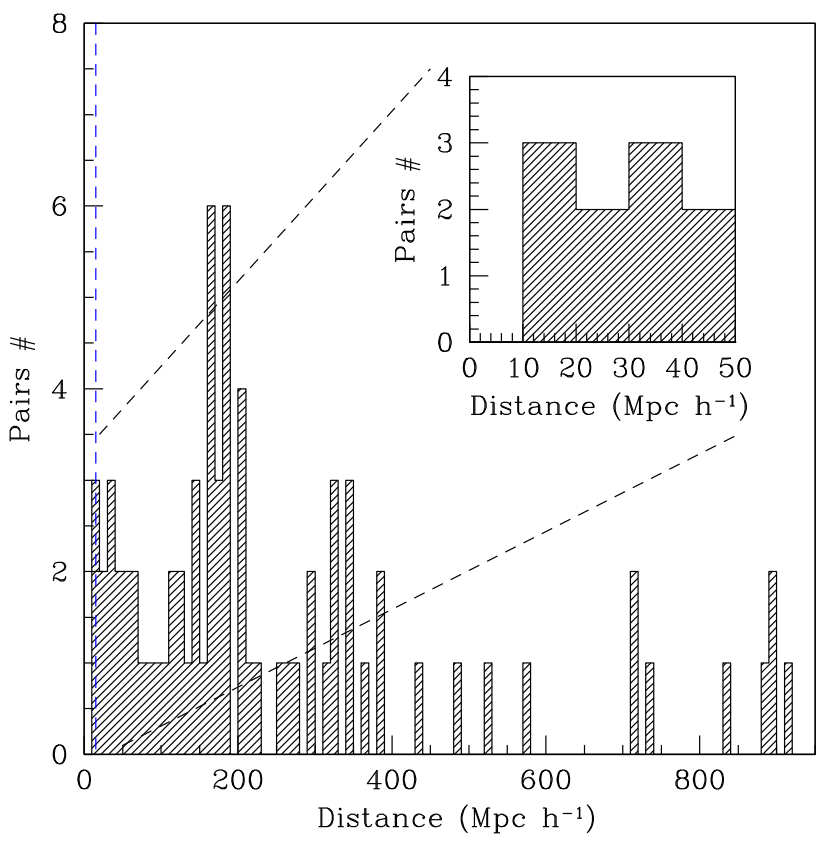

Figure 6. Distribution of 3D distances between all the pairs of clusters with known redshift in the field. The dashed vertical blue line marks a 3D distance of $15 \mathrm{Mpc}^{-1}$. In the inset, a zoom of the region between 0 and $50 \mathrm{Mpch}^{-1}$ is shown.

The two power spectra were then merged with a weighted sum: the single-dish data-weights were set to zero for angular scales smaller than 16 arcmin, to 1 for angular scales larger than 18.3 arcmin, and with a weight that linearly varies from 0 to 1 in between. Vice versa for the interferometric data-weights. The final power spectrum was tapered with the interferometric beam and the data were back-transformed to obtain the combined image. The interferometric and single-dish power spectra are shown in the top panel of Fig. 4, after the beam deconvolution (they have been divided in Fourier space by the Fourier transform of the corresponding beam). The merged power spectrum is shown in the bottom panel of Fig. 4: in the inset the SRT amplitude versus the NVSS amplitude is shown for measurements corresponding to angular scales in the overlapping region. The average of the amplitude of the points in the inset corresponds to the value shown in the large panels. The combined image has a beam of 45 arcsec and a noise $0.45 \mathrm{mJy} \mathrm{beam}^{-1}$, dominated by the NVSS noise. To better highlight the presence of diffuse large-scale sources, we convolved to a beam of 3.5 arcmin $\times 3.5$ arcmin. The resulting combined image is shown in Fig. 5 (grey colours, top panels).

\subsection{Point-source subtraction}

To investigate the presence of large-scale diffuse structures associated with the galaxy clusters reported in Table 1 and with the lowdensity environments connecting them, we performed a subtraction of the point sources with the SCUBE software package (Murgia et al. 2016) in the combined image at 45 arcsec resolution.

The subtraction is done as follows:

(i) the strongest point source in the image is identified and fitted with a non-zero baseline 2D elliptical Gaussian;

(ii) the model is then subtracted from the image;

(iii) the two steps above are repeated until a user-defined threshold is reached.
To take into account the possibility that each source is embedded into large-scale diffuse emission, we model the source with a 2D elliptical Gaussian sitting on a plane. Overall, the free parameters of the fit are nine: the $x, y$ coordinates in the sky of the centre of the Gaussian, the FWHM along the two axis, the position angle, the amplitude, and the three components of the direction normal to the plane. Only in the case of sources weaker than a user-defined threshold (in this case $10 \sigma$ ) the FWHM is forced to assume the value of the beam size. This algorithm has been tested in the context of the work by Fatigoni (2017).

As outputs, the task produces a residual image. We repeated the procedure two times. At first, we used a threshold of about $7 \sigma=3 \mathrm{mJy}_{\text {beam }}-1$, where $\sigma=0.45 \mathrm{mJy}$ beam $^{-1}$ is the noise of the image. As a second step, we again run the algorithm on the residual image, with a lower threshold of $4 \sigma=1.8 \mathrm{mJy}$ beam $^{-1}$. This conservative threshold has been chosen to avoid oversubtraction. In total, 3872 sources have been subtracted. In Fig. 5 (top right and bottom left panels), the resulting residual image is shown in blue contours. In Appendix A, we use a sub-sample of the radio galaxies in the field to cross check the flux scale after the combination of single-dish and interferometric data.

\section{X-RAY EMISSION}

The X-ray properties of the majority of the clusters in the sample are unknown. Information for only a few of the clusters in Table 1 can be found in the literature. The X-ray luminosity of A523, RXC J0506.9+0257, and RXC J0503.1+0607 are reported in the catalogue by Böhringer et al. (2000), while A520 and MACS J0455.2+0657 can be found in Mahdavi et al. (2007) and Mann \& Ebeling (2012), respectively. To our knowledge, no information is available for the remaining clusters.

By applying the approach proposed by Finoguenov et al. (2007), we derived the flux $S_{\mathrm{X}}$ in the $0.5-2 \mathrm{keV}$ energy band and the luminosity $L_{\mathrm{X}}, 0.1-2.4 \mathrm{keV}$ within an extraction area of radius $R_{200}{ }^{3}$ for the clusters in Table 1, by using data from the ROSAT All-Sky Survey (RASS; Voges et al. 1999). The fluxes of the clusters have been corrected for the aperture and for the point spread function and the luminosities have been corrected for the dimming (for details about the procedure please refer to Finoguenov et al. 2007). The values of flux and luminosity of these clusters are reported in Table 4. For the sources with significance of the count rate below $1 \sigma$, the values we provide for all the properties are $2 \sigma$ upper limits.

\section{MILLIMETRE AND SUB-MILLIMETRE EMISSION}

To investigate the properties of the cosmic web in this region of the sky, we superimposed the SRT contours on the Planck image, see Fig. 5 (colours, bottom left panel). The Compton parameter map (hereafter $Y$-map) has been obtained by using the data from the full mission and by applying the Modified Internal Linear Combination Algorithm (Hurier et al. 2013) method. ${ }^{4}$ As described in the work by Planck Collaboration XXII (2016a), this method removes the signal of the cosmic microwave background taking into account its spectral properties.

\footnotetext{
${ }^{3}$ This radius is defined as the radius that encompasses the matter with density 200 times the critical density.

${ }^{4}$ https://www.cosmos.esa.int/web/planck/pla
} 
Table 4. X-ray fluxes of galaxy clusters.

\begin{tabular}{|c|c|c|c|c|c|c|c|}
\hline Cluster & $z$ & $\begin{array}{c}n_{\mathrm{H}} \\
10^{20} \mathrm{~cm}^{-2}\end{array}$ & $\underset{\substack{200 \\
\circ}}{R_{2}}$ & $\begin{array}{c}M_{200} \\
10^{14} \mathrm{M}_{\odot}\end{array}$ & $\begin{array}{c}S_{\mathrm{X}} \\
10^{-12} \mathrm{erg} \mathrm{s}^{-1} \mathrm{~cm}^{-2}\end{array}$ & $\begin{array}{c}D_{\mathrm{L}} \\
\mathrm{Mpc}\end{array}$ & $\begin{array}{c}L_{\mathrm{X},}, 0.1-2.4 \mathrm{keV} \\
10^{43} \mathrm{erg} \mathrm{s}^{-1}\end{array}$ \\
\hline A523 & 0.104 & 10.6 & 0.192 & 2.91 & $1.6 \pm 0.3$ & 499 & $7.3 \pm 1.6$ \\
\hline A525 & 0.1127 & 9.98 & 0.128 & 1.08 & $0.3 \pm 0.1$ & 543 & $1.6 \pm 0.8$ \\
\hline MACSJ0455.2+0657 & 0.425 & 8.41 & 0.096 & 12.87 & $1.3 \pm 0.2$ & 2416 & $116.1 \pm 21.6$ \\
\hline ZwCl0510.0+0458A & 0.027 & 12.0 & $<0.28$ & $<0.19$ & $<0.4$ & 123 & $<0.1$ \\
\hline ZwCl0510.0+0458B & 0.015 & 12.0 & $<0.448$ & $<0.14$ & $<0.7$ & 68 & $<0.1$ \\
\hline RXCJ0503.1+0607 & 0.088 & 8.61 & 0.235 & 3.39 & $2.9 \pm 0.4$ & 418 & $9.0 \pm 1.4$ \\
\hline A529 & 0.1066 & 8.25 & $<0.121$ & $<0.79$ & $<0.2$ & 512 & $<0.9$ \\
\hline A515 & 0.1061 & 8.21 & 0.137 & 1.12 & $0.3 \pm 0.2$ & 509 & $1.6 \pm 0.8$ \\
\hline A526 & 0.1068 & 7.81 & 0.117 & 0.72 & $0.2 \pm 0.1$ & 513 & $0.8 \pm 0.6$ \\
\hline RXCJ0506.9+0257 & 0.1475 & 8.57 & 0.14 & 2.91 & $0.8 \pm 0.2$ & 727 & $7.7 \pm 2.1$ \\
\hline A520 & 0.199 & 5.66 & 0.155 & 8.64 & $2.6 \pm 0.4$ & 1011 & $45.0 \pm 6.1$ \\
\hline A509 & 0.0836 & 8.88 & $<0.133$ & $<0.53$ & $<0.2$ & 395 & $<0.5$ \\
\hline A508 & 0.1481 & 9.85 & 0.118 & 1.75 & $0.4 \pm 0.2$ & 730 & $3.5 \pm 1.5$ \\
\hline
\end{tabular}

Col 1: Cluster name; Col 2: redshift; Col 3: cluster Hydrogen column density; Col 4: $R_{200}$ of the system; Col 5: X-ray flux in the $0.5-2 \mathrm{keV}$ energy band within $R_{200}$; Col 6: mass of the galaxy cluster within $R_{200}$; Col 7: luminosity distance; Col 8: X-ray luminosity in the $0.1-2.4 \mathrm{keV}$ energy band within $R_{200}$.
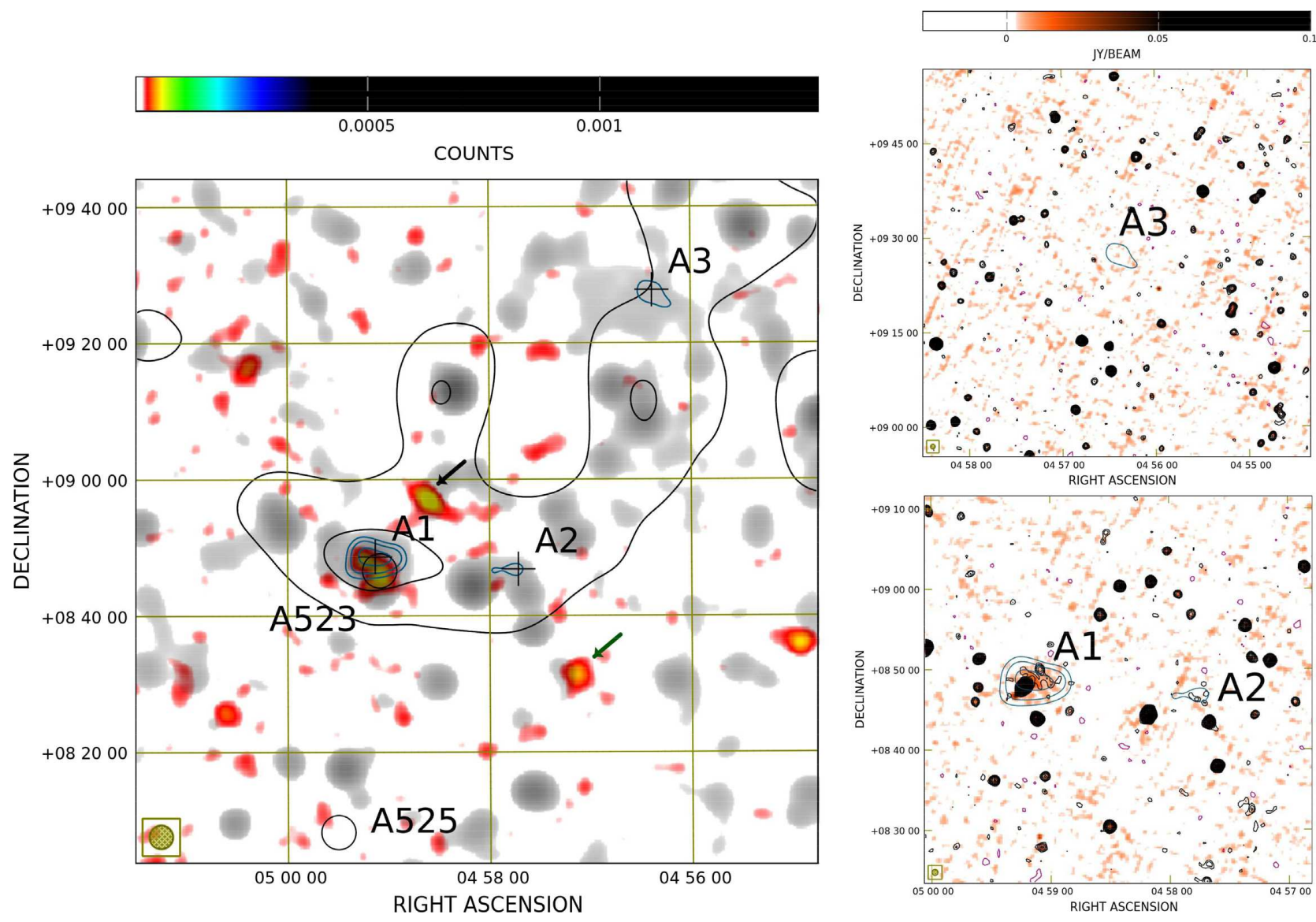

Figure 7. Region A. Left-hand panel: SRT contours $\left(-60,60,150,500 \mathrm{mJy}_{\text {beam }}^{-1}\right.$, negative in magenta and positive in black; resolution 13.9 ar$\mathrm{cmin} \times 12.4 \mathrm{arcmin}$ ) and SRT+NVSS after compact-source subtraction contours (in blue, starting from $3 \sigma$ and the remaining increasing by a factor $\sqrt{2}$; $\sigma=2.5 \mathrm{mJy}_{\text {beam }}{ }^{-1}$, resolution 3.5 arcmin $\times 3.5$ arcmin) overlaid on X-ray emission from the RASS in red colours and radio emission from the SRT+NVSS (resolution 3.5 arcmin) in grey colours. Right-hand panels: NVSS contours $-3 \sigma$ (magenta, $\sigma=0.45 \mathrm{mJy}$ beam ${ }^{-1}$ ), $3 \sigma$, and increasing by a factor $\sqrt{2}$ (black) overlaid on the TGSS in colours. The black and green arrows mark regions where a strong X-ray emission is detected, as described in the text.

Most of the clusters in Table 1 show a counterpart in the Planck image. However, in the catalogue by Planck Collaboration XXVII (2016b) only an association for A520 and one for A539 (RA 05:16:37.3, Dec. +06:26:16, $z=0.0284$ ) within 4 arcmin are present. The strongest signal is detected in the direc- tion of A523 and A520, where it extends well beyond the cluster outskirts. A signal appears to be present also in the direction of A525, A529, RXC J0503.1+0607, MACS J0455.2+0657, RXC J0506.9+0257, A515, A508, A509, and A539 but, unfortunately, the galaxy cluster A539 is at the edge of the SRT field of 

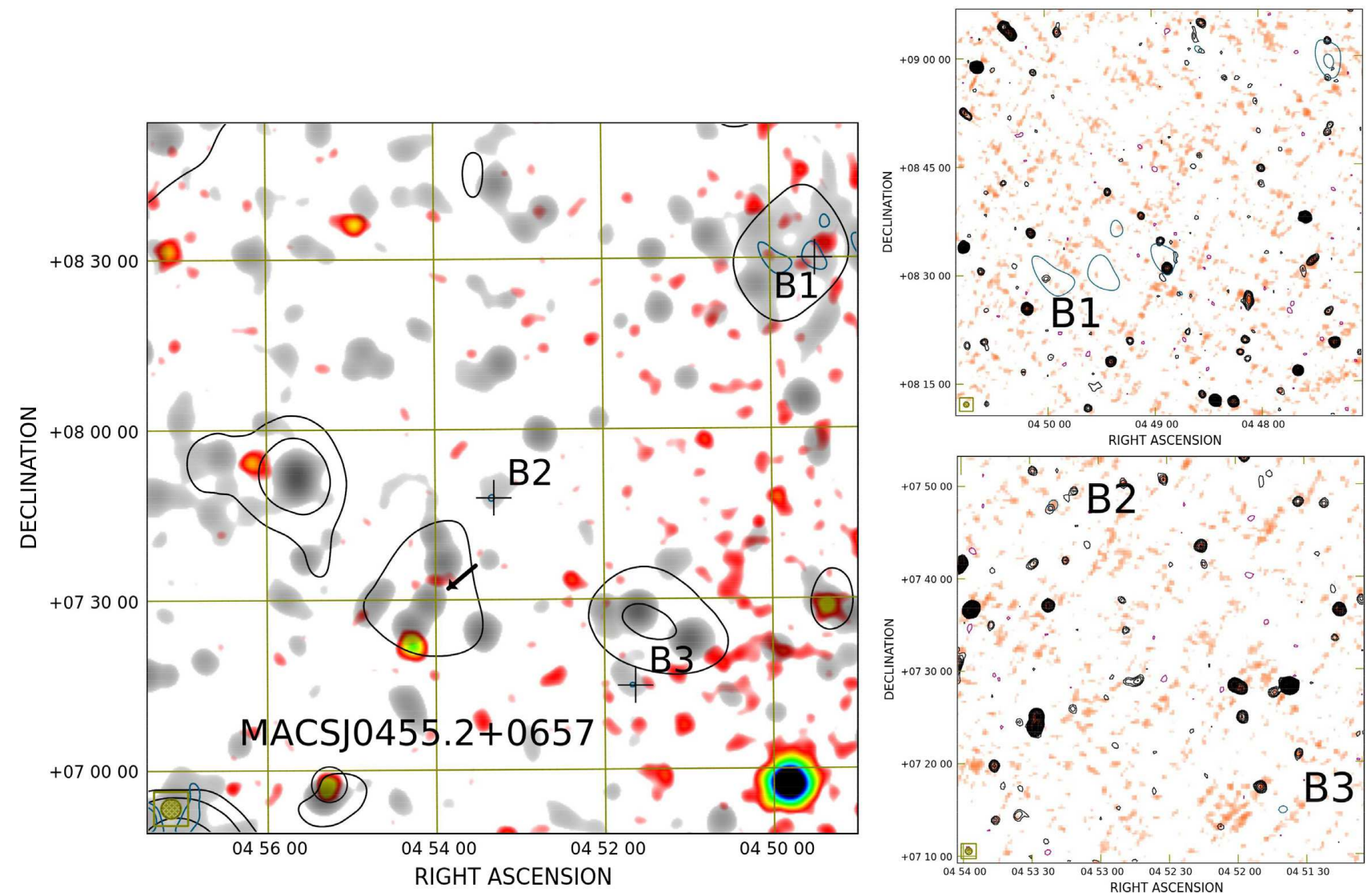

Figure 8. Region B. Left-hand panel: SRT contours $\left(-60,60,150,500 \mathrm{mJy}^{\text {beam }}{ }^{-1}\right.$, negative in magenta and positive in black; resolution 13.9 ar$\mathrm{cmin} \times 12.4 \mathrm{arcmin}$ ) and SRT+NVSS after compact-source subtraction contours (in blue, starting from $3 \sigma$ and the remaining increasing by a factor $\sqrt{2}$; $\sigma=2.5 \mathrm{mJy}_{\text {beam }}{ }^{-1}$, resolution $3.5 \mathrm{arcmin} \times 3.5 \mathrm{arcmin}$ ) overlaid on X-ray emission from the RASS in red colours and radio emission from the SRT+NVSS (resolution 3.5 arcmin) in grey colours. Right-hand panels: NVSS contours $-3 \sigma$ (magenta, $\sigma=0.45 \mathrm{mJy}$ beam $^{-1}$ ), $3 \sigma$, and increasing by a factor $\sqrt{2}$ (black) overlaid on the TGSS in colours. The black arrow marks the region shown in the zoom of Fig. 9. The colorbars are the same as in Fig. 7.

view. On the contrary, for A526 and $\mathrm{ZwCl} 0510.0+0458 \mathrm{~A} / \mathrm{B}$ there is no evidence of a signal above the noise level.

\section{RESULTS}

The SRT contours reveal a field rich of radio sources. This region of the sky is populated by several galaxy clusters starting from the north with the complex A523-A525 down to the south with the complex A508-A509. A large fraction of them are approximately at the same redshift $(\approx 0.1$, see Fig. 1$)$, and possibly form a filament of the cosmic web sitting in the plane of the sky. This hypothesis is supported by the fact that some of them have been observed to be undergoing accretion processes as confirmed by the diffuse large-scale synchrotron emission observed at their centre. This is the case of A523 and A520, even if it is not clear whether A520 belongs to the filament because of its large distance along the line of sight. In the CDM cosmology, clusters of galaxies with a mass exceeding $10^{14}$ $\mathrm{M}_{\odot}$ should have a 80 per cent probability of being connected to a neighbouring cluster by a filamentary joint of dark and gas matter, in case they are less than $15 \mathrm{Mpc} h^{-1}$ apart (Colberg, Krughoff \& Connolly 2005). Based on the X-ray data (Table 4) we can estimate that at least eight of the clusters (A523, A525, MACS J0455.2+0657, RXC J0503.1+0607, A515, RXC J0506.9+0257, A520, and A508) in the field have a mass $M_{200} \geq 10^{14} \mathrm{M}_{\odot}$. Therefore, several of these objects might be connected by filaments difficult to observe through the X-ray/SZ effect but possibly detectable in the radio window. In Fig. 6, we show the distribution of 3D distances between all the pairs of clusters with known redshift in the field. Since an absolute error in the redshift estimate of 0.003 ( 3 per cent) translates in an error in the 3D distance of about $13 \mathrm{Mpc} h^{-1}$, we looked for pairs with 3D distance in the range $0-30 \mathrm{Mpch}^{-1}$. We found five pairs within this range of 3D distance (A526 and RXC J0503.1+0607, A526 and A529, A509 and RXCJ0503.1+0607, A509 and A529, and A509 and A526), suggesting that this field is likely to host filaments of dark and gas matter connecting galaxy clusters.

To identify possible diffuse radio emission in the field and better understand its nature, we subtracted the point sources and compared the residual radio emission with the signal observed at other wavelengths. In the following we present the results and describe the properties of the diffuse radio sources we found.

\subsection{Diffuse emission}

In this section we inspect the SRT, the SRT+NVSS and the residual SRT+NVSS after compact-source subtraction images at $1.4 \mathrm{GHz}$ (see Fig. 5, top right panel) and compare them with X-ray ROSAT data in the $0.1-2.4 \mathrm{keV}$ from the RASS and the SZ Compton parameter from millimetre/sub-millimetre observations obtained with the Planck satellite. 
Table 5. Diffuse large-scale emission properties of sources detected above $3 \sigma$.

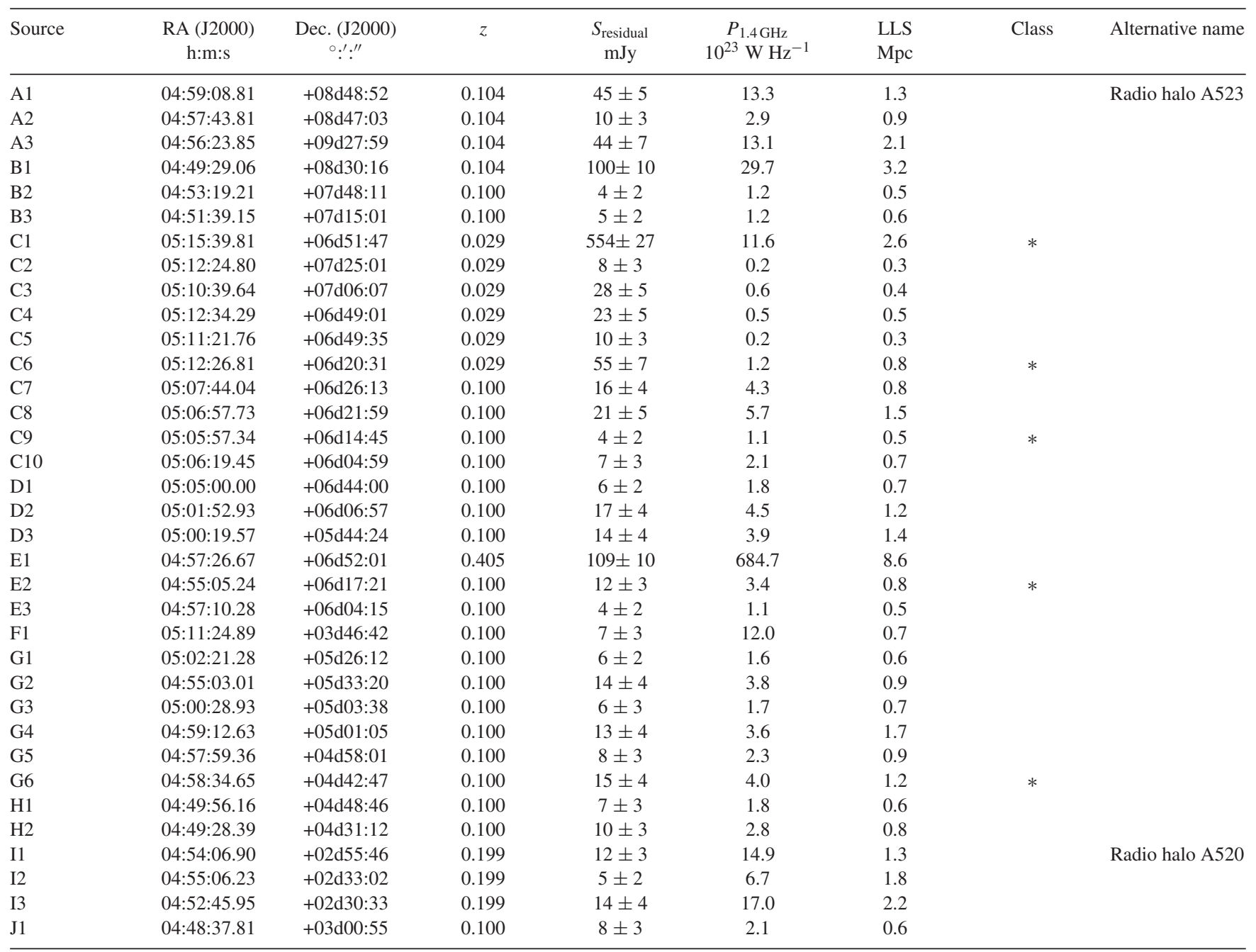

Col 1: Source label; Col 2 and 3: source coordinates; Col 4: redshift; Col 5: flux density at 1.4 GHz of the residual diffuse emission from the SRT+NVSS combined image after the point-source subtraction; Col 6: radio power at 1.4 GHz of the diffuse emission; Col 7: LLS of the diffuse emission; Col 8: an asterisk indicates that the source could be a leftover of compact sources after the subtraction process or an artefact; Col 9: alternative name of the source.

After the subtraction of the compact sources, 35 patches of diffuse synchrotron emission survive, whose locations are shown in Fig. 5 along with the location of the clusters with redshift identification (bottom right panel). The sources are mainly located along the group of clusters with redshift $z \approx 0.1$ (A523, A525, RXC J0503.1+0607, A529, A515, A526, RXC J0506.9+0257, A508, and A509), and in the region around A539 (middle left of the image). We identified eight regions of interest where these 35 candidate sources are located (grey boxes in the bottom left panel of Fig. 5). Due to the large size of the field of view, to better visualize these diffuse radio sources, we present a zoom of each region in figures from Fig. 7 to Fig. 17, labelled respectively with capital letters from A to J (see Fig. 5). In each figure, on the left-hand panel, we overlay the SRT and the SRT+NVSS contours after the point-source subtraction on the X-ray and SRT+NVSS images in colours. On the right-hand panel(s), we show the NVSS contours overlaid on the TIFR GMRT Sky Survey Alternative Data Release (TGSS ADR; Intema et al. 2017) in colours in the left panels. We indicate the position of the clusters with redshift identifications with circles and labels and mark all the spots of diffuse emission with a cross and the letter corresponding to the region of interest plus progressive numbers.
These sources are those visible in the residual image after a $3 \sigma$ cut in radio brightness $\left(\sigma=2.5 \mathrm{mJy}\right.$ beam $\left.^{-1}\right)$. For these sources, we give an estimate of the flux $\left(S_{\text {residual }}\right)$ and the LLS in Table 5. In order to evaluate the integrated flux and the size of these sources, we blanked the convolved residual image at its $2 \sigma$ level. A summary of the radio and X-ray properties of these sources is given in Tables 5 and 6, respectively. Sources at the edge of the image have not been considered because they could be artefacts of the imaging or point-source subtraction process. The only exception is the patch of diffuse emission close-by the galaxy cluster A539. In the following we report a description of the emission observed in each region.

\subsubsection{Region A}

Moving from north to south of the full field of view, the first object is A523 (Fig. 7), which hosts a powerful radio halo (Giovannini et al. 2011) also visible in the NVSS. This system is known to be overluminous in radio with respect to the X-rays. The SRT data indicate a large-scale emission with an elongation to 
Table 6. X-ray fluxes from the regions associated with the diffuse sources in the field from RASS.

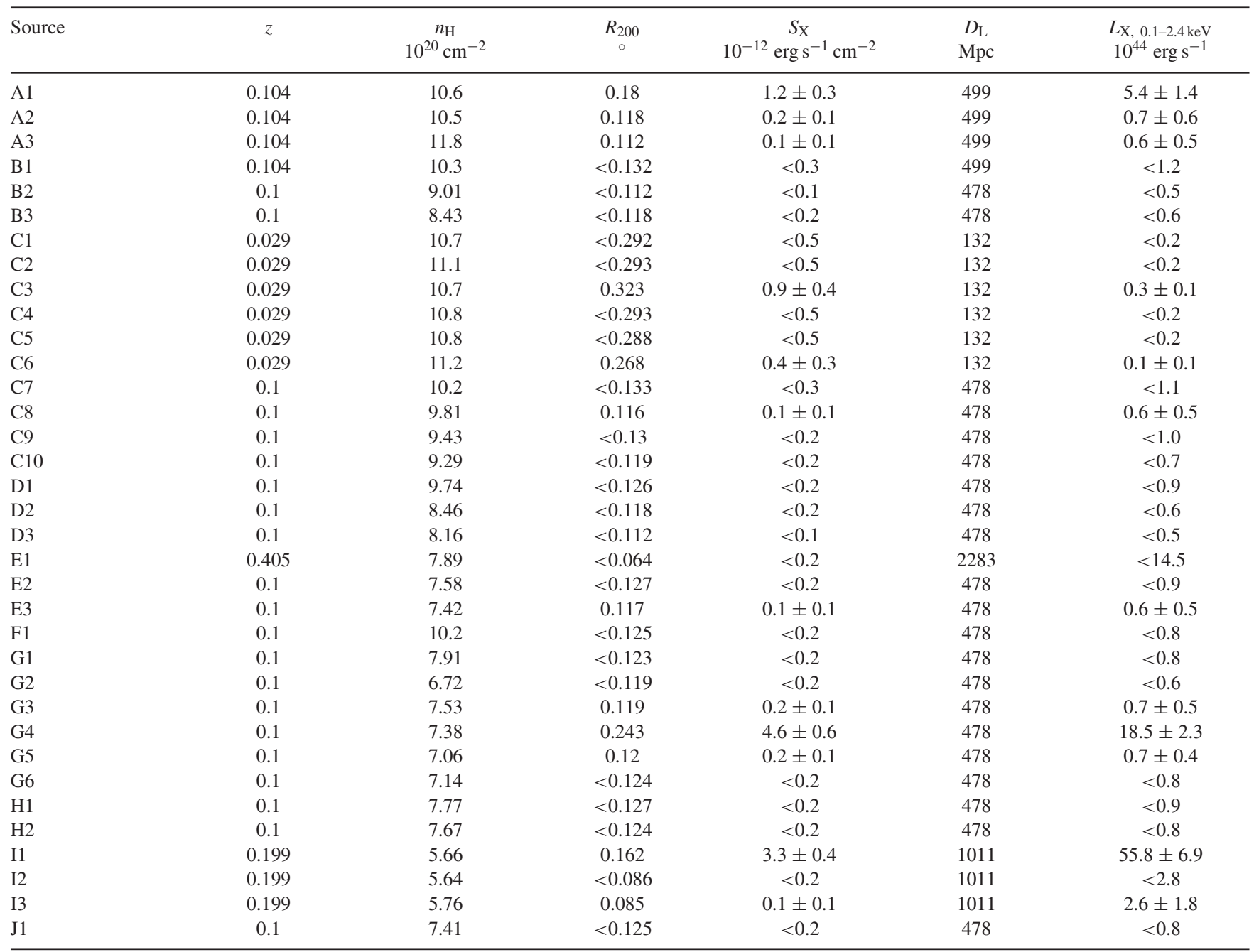

Col 1: Source name; Col 2: redshift; Col 3: Hydrogen column density in the direction of the source; Col 4: $R_{200}$ of the system; Col 5: X-ray flux in the $0.5-2 \mathrm{keV}$ energy band within $R_{200}$; Col 6: luminosity distance; Col 7: X-ray luminosity in the $0.1-2.4 \mathrm{keV}$ energy band within $R_{200}$.

the west of A523. After subtracting the point sources in the combined SRT+NVSS image, a residual diffuse emission emerges at the position of A523 (source A1) and another 12 arcmin (source A2) west of A1. A few patches of diffuse emission can be seen also on the north-west (source A3), where hints of radio emission are already visible in the combined SRT+NVSS image. At the location of sources A2 and A3 no point-source emission is detected in the NVSS image. X-ray emission is clearly visible towards A523 and to the north-west (see black arrow in the image), while only hints of emission are present at the location of A2 and A3. South-west of A2, an X-ray signal is detected (green arrow). By inspecting the high-resolution NVSS and TGSS images in the region corresponding to the X-ray signal, we find hints of diffuse emission. In the SRT+NVSS image after point-source subtraction, a diffuse patch at $2 \sigma$ of significance is detected at the same spatial location.

In the residual image, we measure a flux of the radio halo in A523 of $(44 \pm 5) \mathrm{mJy}$ and a LLS of about $1.2 \mathrm{Mpc}(10 \mathrm{arcmin})$. As a comparison, Giovannini et al. (2011) give a flux $S_{1.4 \mathrm{GHz}}=(59 \pm 5) \mathrm{mJy}$, while Girardi et al. (2016) report $S_{1.4 \mathrm{GHz}}=(72.3 \pm 0.6) \mathrm{mJy}$. The integrated flux measured in this work is slightly weaker than that estimated by Giovannini et al. (2011). This difference can be explained by the different procedures of point-sources subtraction. The corresponding radio power at $1.4 \mathrm{GHz}$ is $1.33 \times 10^{24} \mathrm{~W} \mathrm{~Hz}^{-1}$. The shape and the size of the radio halo in the galaxy cluster A523 from our images are very similar to those previously derived from interferometric observations only. The source appears quite roundish with a slight elongation in the direction perpendicular to the merger axis (SSW-NNE), as derived by X-ray and optical data (Girardi et al. 2016).

\subsubsection{Region $B$}

In Fig. 8, we show the region to the south-west of A523. In the top right corner of the image, an excess of radio emission is detected by the SRT (source B1). Some point sources can be identified in the outskirts of it but not embedded in the emission, excluding the possibility that this source is a blending of point-like sources. Two other diffuse patches of emission can be identified in the field (source B2 and B3), not clearly associated with point-like sources but rather located at positions where an excess in radio emission is observed in the combined SRT+NVSS image (refer to Macario et al. 2014, for a similar case). At the location of Source B2 a 


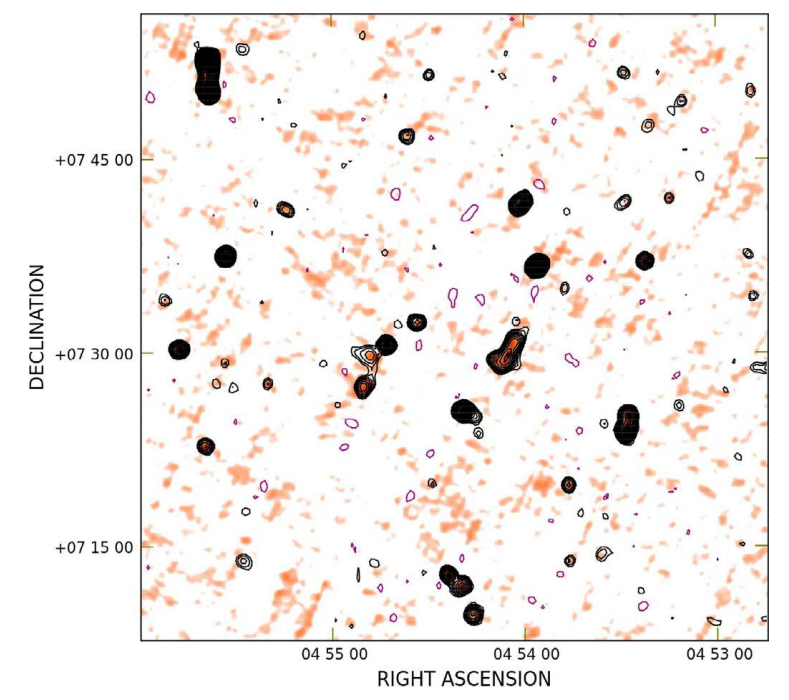

Figure 9. Zoom of the central portion of Region B (see arrow in Fig. 8). The image shows the NVSS contours $\left(-1.35,1.35 \mathrm{mJy}_{\text {beam }}{ }^{-1}\right.$, and the remaining scaled by a factor $\sqrt{2}$, negative in magenta and positive in black) overlaid on the TGSS colours. The colorbar is the same as in the right-hand panels of Fig. 7.

hint of diffuse emission is observed also in the NVSS and TGSS images.

According to the literature, no galaxy cluster is present in the field shown in Fig. 8. However, high-resolution NVSS and TGSS data (Fig. 9) show that the emission detected by the SRT at the centre of the panel, south-east of B2, is a blending of compact radio sources that might be part of a galaxy cluster. In a region of radius 20 arcmin centred on the radio galaxy indicated by the arrow in Fig. 8, only the source 2MASX J04534530+0719303 located at RA 04h:53m:45.3s and Dec. $+07 \mathrm{~d}: 19 \mathrm{~m}: 31 \mathrm{~s}$ has a spectroscopic redshift measurement $(z=0.104$; Rines et al. 2003). These galaxies could be part of a galaxy cluster or a group of galaxies at the same redshift of the filament.

\subsubsection{Region C}

East of the filament, another region of interest can be identified (see Fig. 10) on the west of the galaxy cluster A539, whose position is indicated by the arrow in the same figure. The cluster appears to be an emitter in the X-ray and millimetre/sub-millimetre domain but, unfortunately, it is outside the field of view of our SRT observations and only the region to its west has been mapped. In the SRT image, we see a large arm extending from the cluster westward, still visible in the residual image as a large diffuse structure. This structure cannot be entirely explained as the blending of radio galaxies in the field, so it is likely related to a large-scale diffuse source. After the subtraction of point sources, ten patches (Sources from $\mathrm{C} 1$ to $\mathrm{C} 10)$ remain. $\mathrm{C} 6$ and $\mathrm{C} 9$ could be the leftovers of the point-like source subtraction process as well as $\mathrm{C} 1$ at least partially, while the remaining sources seem to be large-scale diffuse synchrotron sources. The largest and more interesting patch is C1. Hints of diffuse large-scale emission at the same spatial location are present in the SRT+NVSS and in the X-ray image. A slight excess of largescale radio emission can be identified as well at higher resolution in the NVSS. However, since it is located at the edge of the image, more observations are required to confirm it and investigate its nature.

\subsubsection{Region D}

The complex RXC J0503.1+0607-A529 is located at the centre of the panel shown in Fig. 11 and the galaxy cluster $\mathrm{ZwCl} 0459.6+0606$ sits between them (see Table 2). In the bottom right corner of the image, the galaxy cluster A526 is also partially visible. In the X-rays, only RXC J0503.1+0607 shows a signal above the noise level. In radio, both RXC J0503.1+0607 and A529 can be seen: RXC J0503.1+0607 is a strong emitter, while A529 is characterized by a faint signal. The peak observed in the SRT image between the two clusters is approximately at the same angular location as $\mathrm{ZwCl} 0459.6+0606$ (see arrow in Fig. 11) and is due to the quasar PKS 0459+060 (RA 05h:02m:15.4s, Dec. +06d:09m:07s, $z=1.106)$. The superposition of the SRT and the SRT+NVSS images, reveals the presence of several point sources in the field, as well as hints of large-scale emission in between the two clusters. This indication is confirmed by the residual image, where patches of large-scale diffuse emission are present (D1, D2, and D3). Source D1 in the top left corner is located in a region where the SRT+NVSS image reveals a large-scale patch of radio emission. Source D2 does not show a connection with point-like sources in the field and appear located at the periphery of $\mathrm{ZwCl} 0459.6+0606$. Indications of the presence of a large-scale signal at locations of D1 and D2 can be seen in the NVSS image. A puzzling patch of diffuse emission is observed to the south-west (source D3), in the outskirts of A526, without a clear association with any point sources but rather corresponding to hints of diffuse emission in the SRT+NVSS images.

\subsubsection{Region $E$}

In Fig. 12, another interesting complex can be identified $2^{\circ}$ south of A523, south-east of the galaxy cluster MACS J0455.2+0657. In this region, MACS J0455.2+0657 is the only galaxy cluster with a spectroscopic redshift measurement $(z=0.425)$. In the field of view of Fig. 12, the SRT image reveals three bright peaks (see black arrows in Fig. 12) and a faint large-scale emission in between them (red arrow). At higher resolution, several point sources can be identified. Three of them have the same spatial location as the three peaks detected by the SRT: $4 \mathrm{C}+06.21$ (RA 04h:57m:07.7099s, Dec. +06d:45m:07.260s, $z=0.405$, Drinkwater et al. 1997), PKS 0456+060 (RA 04h:58m:48.782s, Dec. $+06^{\circ}: 08^{\prime}: 04.26^{\prime \prime}, z=1.08$, Veron-Cetty \& Veron 1996), and LQAC 073+006001 (RA 04h:55m:03.1s, Dec. +06 :24':54", no redshift identification). After point-source subtraction, three interesting large-scale patches emerge. One off the bright radio source $4 C+06.21$ (source E1), one some 10' south of LQAC 073+006 001 (source E2), and one south of the central radio emission (source E3), where no emission appears in the SRT image above the noise level. The source E1 does not appear to be a blending of point-like sources as shown by the superposition with higher resolution data in Fig. 12 and hints of large-scale diffuse emission are already present in the NVSS image. E1 is characterized by an average brightness of $\approx 0.13 \mu \mathrm{Jy} \operatorname{arcsec}^{-2}$, its integrated flux density is $(109 \pm 10) \mathrm{mJy}$, and its largest angular size is 25 arcmin. If we assume that this emission is at the same redshift as the radio source $4 \mathrm{C}+06.21(z$ $=0.405)$, its LLS is $8.7 \mathrm{Mpc}$ and its radio power at $1.4 \mathrm{GHz}$ is $6.8 \times 10^{25} \mathrm{~W} \mathrm{~Hz}^{-1}$. However, if we assume that this source is located at the average redshift of the filament $(z \approx 0.1)$, its LLS is about $3 \mathrm{Mpc}$ and its radio power at $1.4 \mathrm{GHz}$ is $3.0 \times 10^{24} \mathrm{~W} \mathrm{~Hz}^{-1}$. No strong X-ray emission is present in the field except that seen to the north-east of the radio source E1. Source E3 is located at 

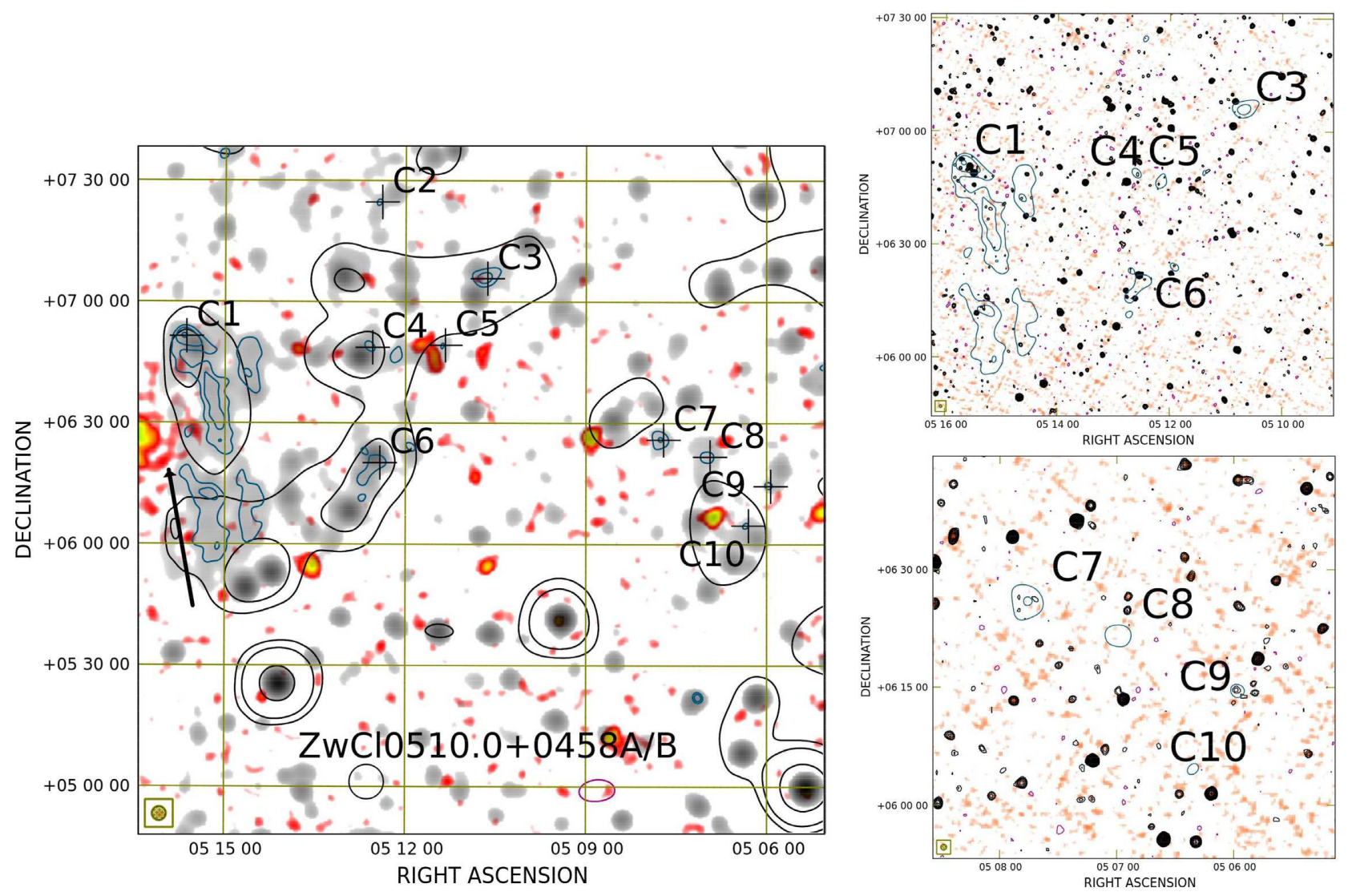

Figure 10. Region C. Left-hand panel: SRT contours $\left(-60,60,150,500 \mathrm{mJy}_{\text {beam }}^{-1}\right.$, negative in magenta and positive in black; resolution 13.9 ar$\mathrm{cmin} \times 12.4$ arcmin) and SRT+NVSS after compact-source subtraction contours (in blue, starting from $3 \sigma$ and the remaining increasing by a factor $\sqrt{2}$; $\sigma=2.5 \mathrm{mJy} \mathrm{beam}^{-1}$, resolution $3.5 \mathrm{arcmin} \times 3.5 \mathrm{arcmin}$ ) overlaid on X-ray emission from the RASS in red colours and radio emission from the SRT+NVSS (resolution 3.5 arcmin) in grey colours. Right-hand panels: NVSS contours $-3 \sigma$ (magenta, $\sigma=0.45 \mathrm{mJy} \mathrm{beam}^{-1}$ ), $3 \sigma$, and increasing by a factor $\sqrt{2}$ (black) overlaid on the TGSS in colours. The black arrow marks the position of A539. The colorbars are the same as in Fig. 7.

position where the combined NVSS indicates the presence of an excess of large-scale diffuse emission, while the source E2 could be the residual of point-like emission. At the centre of the panel, at coordinates RA 04h:56m:25.24s, Dec. $+06^{\circ}: 13^{\prime}: 29^{\prime \prime}$ (see green arrow), a $2 \sigma$-level signal is observed in the residual radio image. Since it is below the $3 \sigma$ significance threshold, we did not consider it in our analysis. However, at the same spatial location, a faint X-ray emission and an SZ signal (see Fig. 5) are present, suggesting that these emissions could be real and not a statistical fluctuation.

\subsubsection{Region F}

In the region shown in Fig. 13 no galaxy cluster is identified in the literature. The field is rich in point-like radio sources as shown by the SRT+NVSS image. The SRT+NVSS image after point-source subtraction shows five patches of diffuse emission. The two on the left-hand side are not considered here, since they are at the edge of the image and might be artefacts. The two spots in the top right region reveal an extended radio galaxy (see arrow in the image) that we classify as a candidate new giant radio galaxy and discuss in Appendix B. Our interest here is the source F1 located at the centre of the image. This source is located close-by a point-like source, spatially coincident with a fainter large-scale region visible also in the NVSS image.

\subsubsection{Region $G$}

The galaxy cluster A526 is shown in the top of Fig. 14. The cluster is visible in radio and X-rays, but shows no counterpart at millimetre/sub-millimetre wavelengths. To the south-west, large-scale emission is detected with the SRT. No galaxy clusters have been identified in this region apart from the galaxy cluster $\mathrm{ZwCl} 0457.0+0511$ (see black arrow in the image), south-west of A526 at an angular offset of about 10 arcmin. Two strong X-ray sources are seen: one south of A526, approximately coincident with the radio source PMN J0459+0455 (RA 04h:59m:04.7s, Dec. $+04 \mathrm{~d}: 55 \mathrm{~m}: 59 \mathrm{~s}$, see red arrow) and the other in the west with no obvious radio counterpart. After subtracting the point-source emission, several patches of diffuse emission emerge. Sources G1 and $\mathrm{G} 2$, respectively in the top left and right corners, have the same spatial location of an excess in the NVSS image. Another patch of residual radio emission is present in the north-east of the image (source G3), close-by to a point source surrounded by a fainter large-scale signal and where the SRT image shows a bridge of radio emission (see green arrow) connecting A526 with an overdensity of radio galaxies south-east of A526. At the centre of the panel, three patches of diffuse emission are present corresponding to the large-scale central SRT radio emission: sources G4, G5, G6. Sources G4 and G5 appear to be diffuse sources, while source G6 could be a residual of the point source located below. 

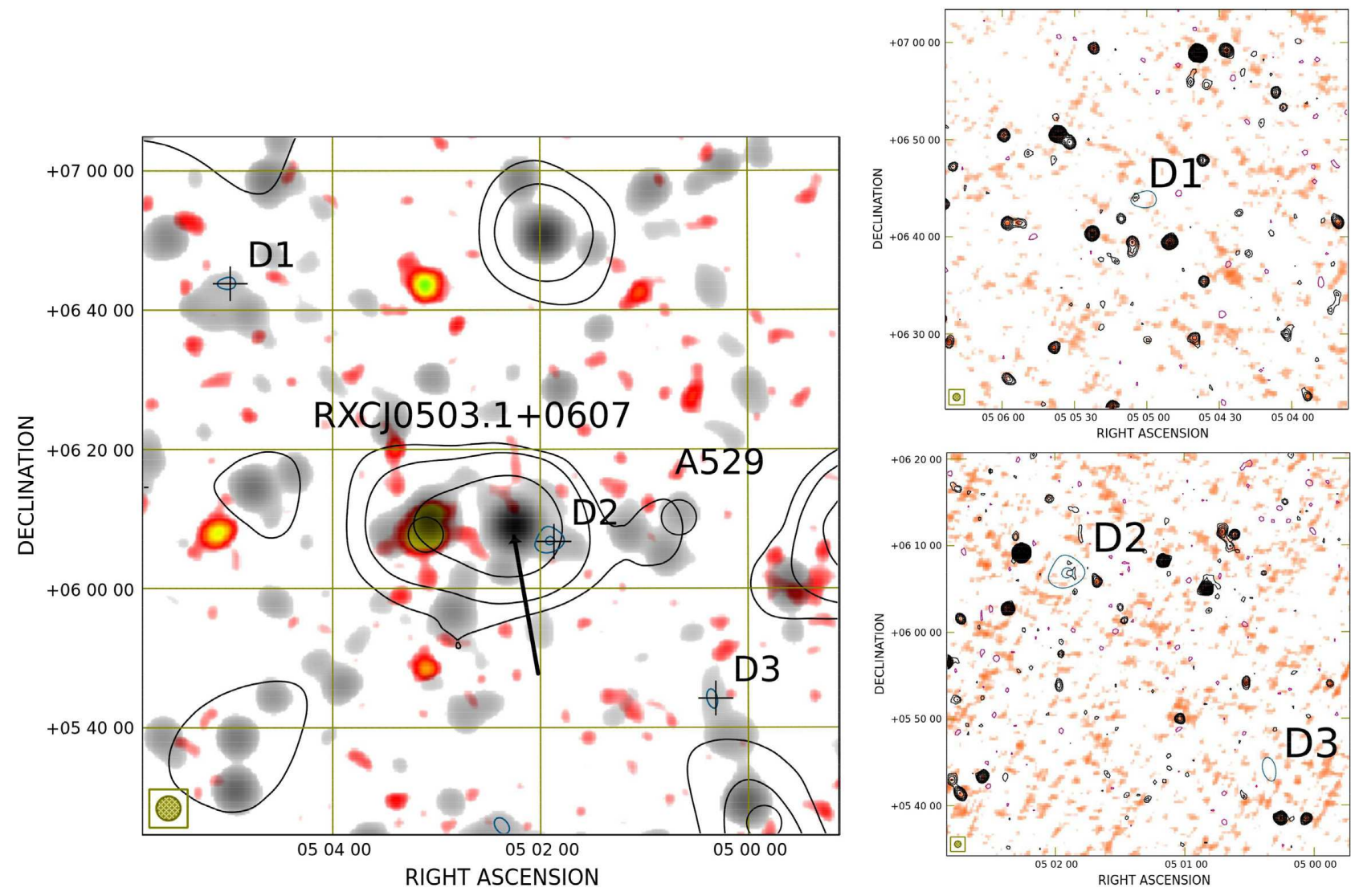

Figure 11. Region D. Left-hand panel: SRT contours $\left(-60,60,150,500 \mathrm{mJy}_{\text {beam }}^{-1}\right.$, negative in magenta and positive in black; resolution 13.9 ar$\mathrm{cmin} \times 12.4 \mathrm{arcmin}$ ) and SRT+NVSS after compact-source subtraction contours (in blue, starting from $3 \sigma$ and the remaining increasing by a factor $\sqrt{2}$; $\sigma=2.5 \mathrm{mJy}_{\text {beam }}{ }^{-1}$, resolution 3.5 arcmin $\times 3.5$ arcmin) overlaid on X-ray emission from the RASS in red colours and radio emission from the SRT+NVSS (resolution $3.5 \mathrm{arcmin}$ ) in grey colours. Right-hand panels: NVSS contours $-3 \sigma$ (magenta, $\sigma=0.45 \mathrm{mJy}_{\text {beam }}^{-1}$ ), $3 \sigma$, and increasing by a factor $\sqrt{2}$ (black) overlaid on the TGSS in colours. The black arrow marks the position of the quasar PKS 0459+060. The colorbars are the same as in Fig. 7.

\subsubsection{Region $H$}

This region (Fig. 15) is full of point-like sources as shown by the SRT+NVSS image. In the SRT image a central patch likely due to the blending of point-like sources is present (see arrow in the image). Above and below this emission, two patches of residual radio signal (sources $\mathrm{H} 1$ and $\mathrm{H} 2$ ) are identified that do not appear to be directly linked to point-like sources. In particular, at the location of $\mathrm{H} 1$, an indication of diffuse emission is detected also in the NVSS image.

\subsubsection{Region I}

At the centre of Fig. 16 the galaxy cluster A520 is present, located in the south of the full field of view. Strong X-ray emission is spatially coincident with this cluster that appears to be very bright at millimetre/sub-millimetre wavelengths (see Fig. 5). Hints of diffuse emission are present in the NVSS in the direction of A520 (where indeed a radio halo is present), east, south and south-west of the cluster. After the point-source subtraction in the SRT+NVSS image, patches of diffuse large-scale emission remain at these locations and the radio halo in A520 (source I1) is now clearly visible. In the residual image blanked at the $2 \sigma$ level, we measure an integrated flux of $(12 \pm 3) \mathrm{mJy}$ and an LLS of about $1 \mathrm{Mpc}$ for this radio halo. The corresponding radio power at $1.4 \mathrm{GHz}$ is $1.48 \times 10^{24} \mathrm{~W} \mathrm{~Hz}^{-1}$. As a comparison, the values reported in the literature are $(16.7 \pm 0.6) \mathrm{mJy}$ and 1.05 Mpc (Govoni et al. 2001; Vacca et al. 2014) which are very consistent with ours. South of A520, the sources I 2 and I3 can be observed. They do not show a clear link with radio galaxies in the field, on the contrary they are located where hints of diffuse radio emission are present in the NVSS image, so they could be real largescale diffuse synchrotron sources. The present observations of the radio halo in the galaxy cluster A520 confirm what was suggested by the data at higher resolution. The source is quite complex and with a strong elongation in the NE-SW direction. The peak of the emission is located in the SW at the same location as the shock front (Markevitch et al. 2005; Govoni et al. 2004), where a sharp drop in radio surface brightness is detected. A faint tail of radio emission is left behind in the cool region. As already suggested by Govoni et al. (2001) and Vacca et al. (2014), this peculiar source could actually be a peripheral source seen in projection.

\subsubsection{Region $J$}

The complex A508-A509 sits in the south-west corner of the full field of view and is shown in Fig. 17. The two clusters are also visible in X-rays and at millimetre/sub-millimetre wavelengths but they are quite faint. Patches of diffuse emission not coincident with pointlike sources survive in the residual image. Source J1 is located where an excess of radio emission is observed in the NVSS image. The other patches are located at the edge of the image and are therefore hard to discriminate if they are real or artefacts, even though the 

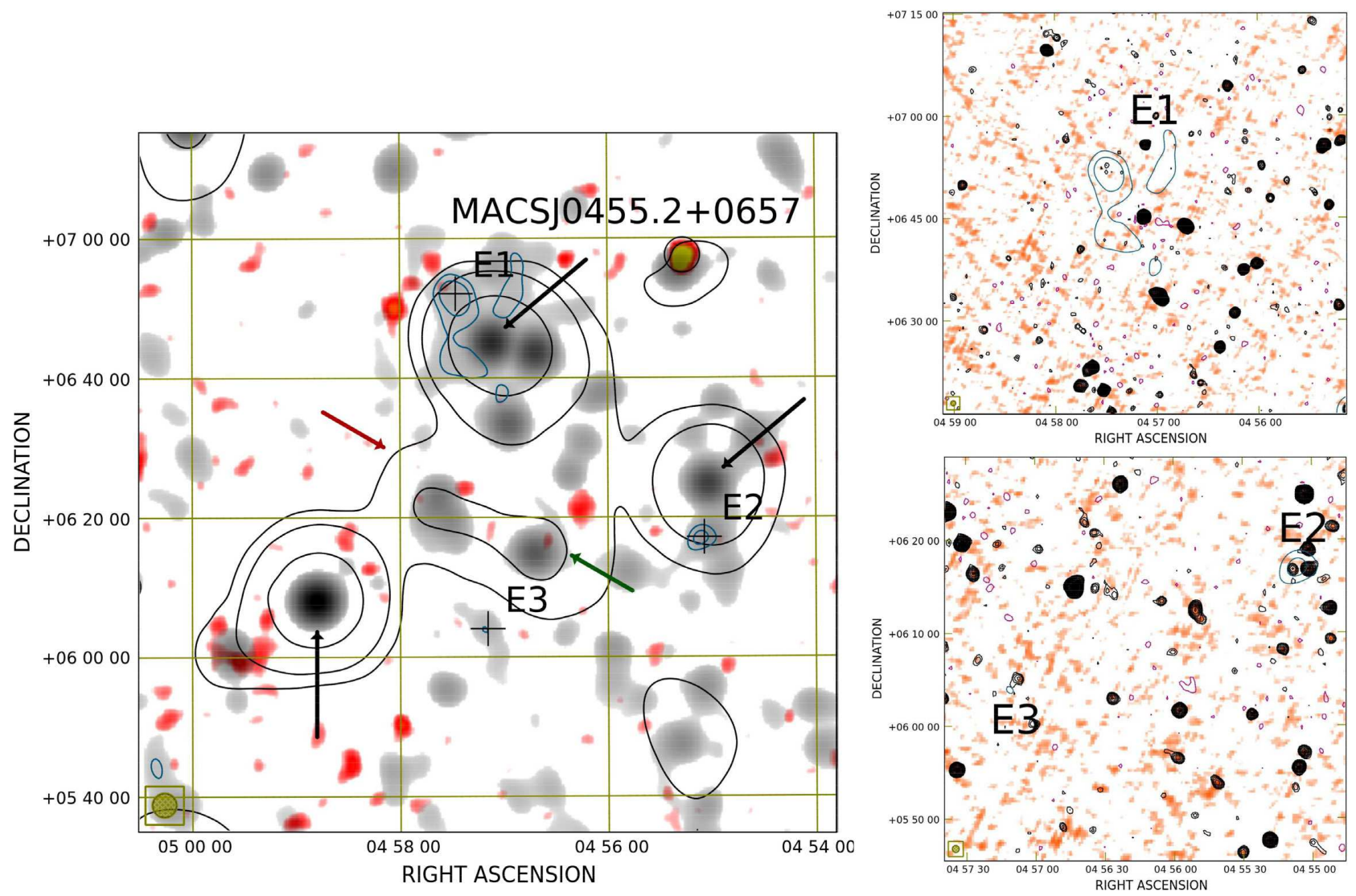

Figure 12. Region E. Left-hand panel: SRT contours $\left(-60,60,150,500 \mathrm{mJy}_{\text {beam }}^{-1}\right.$, negative in magenta and positive in black; resolution 13.9 ar$\mathrm{cmin} \times 12.4$ arcmin) and SRT+NVSS after compact-source subtraction contours (in blue, starting from $3 \sigma$ and the remaining increasing by a factor $\sqrt{2}$; $\sigma=2.5 \mathrm{mJy} \mathrm{beam}^{-1}$, resolution $3.5 \mathrm{arcmin} \times 3.5 \mathrm{arcmin}$ ) overlaid on X-ray emission from the RASS in red colours and radio emission from the SRT+NVSS (resolution 3.5 arcmin) in grey colours. Right-hand panels: NVSS contours $-3 \sigma$ (magenta, $\sigma=0.45 \mathrm{mJy} \mathrm{beam}^{-1}$ ), $3 \sigma$, and increasing by a factor $\sqrt{2}$ (black) overlaid on the TGSS in colours. The black arrows mark the position of 4C+06.21, PKS 0456+060, and LQAC 073+006 001, and the red arrow the faint large-scale SRT emission in between these three bright compact sources. The green arrow marks the location where signal is clearly visible in the SZ and X-ray images while in the SRT+NVSS after compact-source subtraction is detected only with a $2 \sigma$ significance. The colorbars are the same as in Fig. 7.

SRT+NVSS image clearly shows a patch of diffuse emission before compact-source subtraction.

\section{DISCUSSION}

After subtracting the point-source emission, we identified 35 patches of diffuse synchrotron emission in the residual SRT+NVSS image. Two of them are the radio halos in the galaxy clusters A520 and A523, and five are probably artefacts or the leftover of point-like sources after the point-source subtraction process. The remaining 28 are potentially new real large-scale diffuse synchrotron sources, possibly associated with the large-scale structure of the cosmic web. The bottom right panel of Fig. 5 shows that most of the new sources presented in this paper lie along the filament connecting the clusters from A523-A525 in the north to A508-A509 in the south, with an overdensity at the centre of the field of view, at the location of RXC J0503.1+0607, A529 and A526. In the following, we restrict our analysis to the properties of the 28 new detections and the two radio halos already known. We excluded sources that could be artefacts or a remnant of compact sources after the subtraction procedure. To better investigate their properties, we measured their radio powers and sizes and their X-ray luminosities, summarized in Tables 5 and 6, respectively. The X-ray fluxes have been estimated by following the procedure described in Sec- tion 5. Only eleven sources have a significance of the X-ray count rate above $1 \sigma$. These sources are A1 (diffuse emission in A523), A2, A3, C3, C8, E3, G3, G4, G5, I1 (diffuse emission in A520), and $\mathrm{I} 3$.

Some of the sources presented here are potentially interesting and complex systems. For example, source A2 is a faint large-scale source $10^{\prime}$ west of the radio halo in A523 (source A1). It has a radio flux at $1.4 \mathrm{GHz} S_{\text {residual }}=(9 \pm 3) \mathrm{mJy}$ $\left(P_{1.4 \mathrm{GHz}}=2.8 \times 10^{23} \mathrm{~W} \mathrm{~Hz}^{-1}\right)$, an LLS of $1.3 \mathrm{Mpc}$, and an X-ray luminosity $L_{\mathrm{X}, 0.1-2.4 \mathrm{keV}}=(0.7 \pm 0.6) \times 10^{44} \mathrm{erg} \mathrm{s}^{-1}$. In the south-west of A2, another diffuse patch of synchrotron emission is detected at $2 \sigma$ significance and at the same spatial location of an X-ray signal (see Section 7.1.1). These three sources could form an arc-shaped filament of diffuse synchrotron emission. If A2 and the source southwest of it are interpreted to be radio halos, Source A1-Source A2 and this third source could represent the first case of a triple radio halo, which would be particularly interesting because of its association with an underluminous X-ray system. To, date only one other multiple system is known, namely the double radio halo discovered by Murgia et al. (2010) in the galaxy cluster couple A399-A401. In that case the two clusters are about $2.4 \mathrm{Mpc}$ apart, while in this case the distance between these sources is about $1.4 \mathrm{Mpc}$ if all of them are at the same redshift of Source A1 $(z=0.104)$. A follow up at the Xray and optical frequencies is needed to understand if at this location 

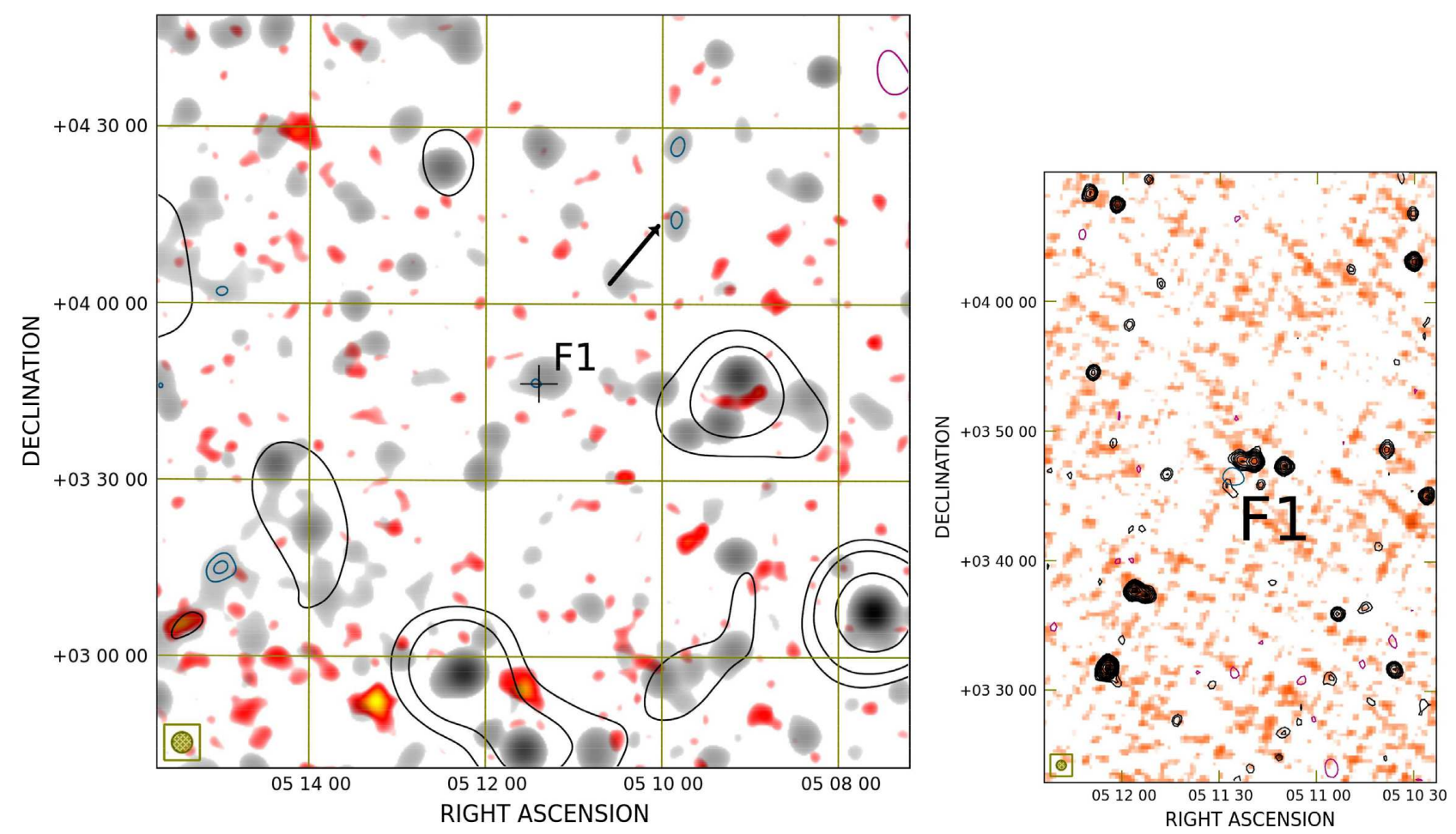

Figure 13. Region F. Left-hand panel: SRT contours $\left(-60,60,150,500 \mathrm{mJy}^{-1}\right.$ beam $^{-1}$, negative in magenta and positive in black; resolution 13.9 arcmin $\times 12.4$ arcmin) and SRT+NVSS after compact-source subtraction contours (in blue, starting from $3 \sigma$ and the remaining increasing by a factor $\sqrt{2}$; $\sigma=2.5 \mathrm{mJy}_{\text {beam }}{ }^{-1}$, resolution $3.5 \mathrm{arcmin} \times 3.5 \mathrm{arcmin}$ ) overlaid on X-ray emission from the RASS in red colours and radio emission from the SRT+NVSS (resolution 3.5 arcmin) in grey colours. Right-hand panel: NVSS contours $-3 \sigma$ (magenta, $\sigma=0.45 \mathrm{mJy}$ beam ${ }^{-1}$ ), $3 \sigma$, and increasing by a factor $\sqrt{2}$ (black) overlaid on the TGSS in colours. The black arrow marks the position of the candidate giant radio galaxy we found (see Appendix B). The colorbars are the same as in Fig. 7.

other galaxy clusters are present and shed light on the nature of these sources.

An even more mysterious source is the emission we label E1. This source appears to be exactly along the long filament connecting A523-A525 in the north to A509-A508 in the south-west, and in the same direction of the radio galaxy $4 \mathrm{C}+06.21$, embedded in the diffuse emission and sitting at redshift $z=0.405$. There is no indication in the literature of a galaxy cluster at the same location in the sky and the closest galaxy clusters to the source are MACS J0455.2+065 $\left(\approx 25^{\prime}\right)$ and A529 (at about $\left.50^{\prime}\right)$. Therefore, Source E1 could be either associated with the filament or a diffuse large-scale structure in the background, as suggested by the redshift of the closest radio source. Source E1 is the most luminous and most extended source in the sample, with a flux at $1.4 \mathrm{GHz} S_{\text {residual }}=(109 \pm 10) \mathrm{mJy}$ $\left(P_{1.4 \mathrm{GHz}}=6.8 \times 10^{25} \mathrm{~W} \mathrm{~Hz}\right)$, an LLS of $8.7 \mathrm{Mpc}$, assuming it is at the same redshift of $4 \mathrm{C}+06.21(z=0.405)$. On the other hand, if we assume that the source is at the same redshift of the filament $(z \approx$ 0.1 ), we derive a radio power $P_{1.4 \mathrm{GHz}}=3.0 \times 10^{24} \mathrm{~W} \mathrm{~Hz}^{-1}$ and an LLS $=3 \mathrm{Mpc}$, still among the brightest and largest sources of the sample. Nevertheless, the source is very faint in the X-ray, for which we derive a luminosity $L_{\mathrm{X}}, 0.1-2.4 \mathrm{keV}<14.5 \times 10^{44} \mathrm{erg} \mathrm{s}^{-1}(2 \sigma)$ if a redshift $z=0.405$ is taken. A firm classification is not possible without further information about the system over a wide range of frequencies, from the X-ray to optical and radio bands.Overall, the nature of the above sources remains unclear. To investigate if they are similar to diffuse cluster sources (i.e. radio halos and relics, e.g. Feretti et al. 2012) or rather they represent a different population, we compare the radio power, radio size, and X-ray luminosity of these new sources with those of known diffuse cluster sources. Hereafter, we adopt for known cluster sources the values given by Feretti et al. (2012) without applying any correction for the cosmology. In Fig. 18, we show in the left-hand panel the radio power at $1.4 \mathrm{GHz}$ $P_{1.4 \mathrm{GHz}}$ versus the X-ray luminosity $L_{\mathrm{X}}, 0.1-2.4 \mathrm{keV}$ in the energy band $0.1-2.4 \mathrm{keV}$ and in the right-hand panel the radio power at $1.4 \mathrm{GHz}$ $P_{1.4 \mathrm{GHz}}$ versus the largest linear size LLS at $1.4 \mathrm{GHz}$, for cluster sources (radio halos and radio relics) from Feretti et al. (2012) and for the new sources presented in this work. For the diffuse emission in A523 and A520 (Sources A1 and I1), we plot both the values we derive and the values found in the literature in order to have a basis for comparison. Our values are slightly different than the values available in the literature ${ }^{5}$, as discussed in Section 7.1, but still follow the correlation between radio and X-ray properties for known diffuse radio sources. Among the eleven sources in our catalogue with a significance of the X-ray count rate above $1 \sigma$, two are the radio halos in A520 and A523, one (G4) follows the correlation observed for radio halos and relics, and the remaining eight show an X-ray luminosity between 10 and 100 times lower than expected from their radio power given the correlation observed for cluster sources, with an average X-ray luminosity of $L_{\mathrm{X}, 0.1-2.4 \mathrm{keV}}=8.4 \times$ $10 \mathrm{erg} \mathrm{s}^{-1}$. They populate a new region of the $\left(L_{\mathrm{X}}, 0.1-2.4 \mathrm{keV}, P_{1.4 \mathrm{GHz}}\right)$ plane that was previously unsampled. The remaining sources are

${ }^{5}$ We note that the discrepancy in the radio power of the halo in A520 is due to the fact that Feretti et al. (2012) report the value given by Govoni et al. (2001). A re-analysis of the radio halo properties was performed by Vacca et al. (2014) that find a radio power consistent with our measurement, as described in Section 2.1. 

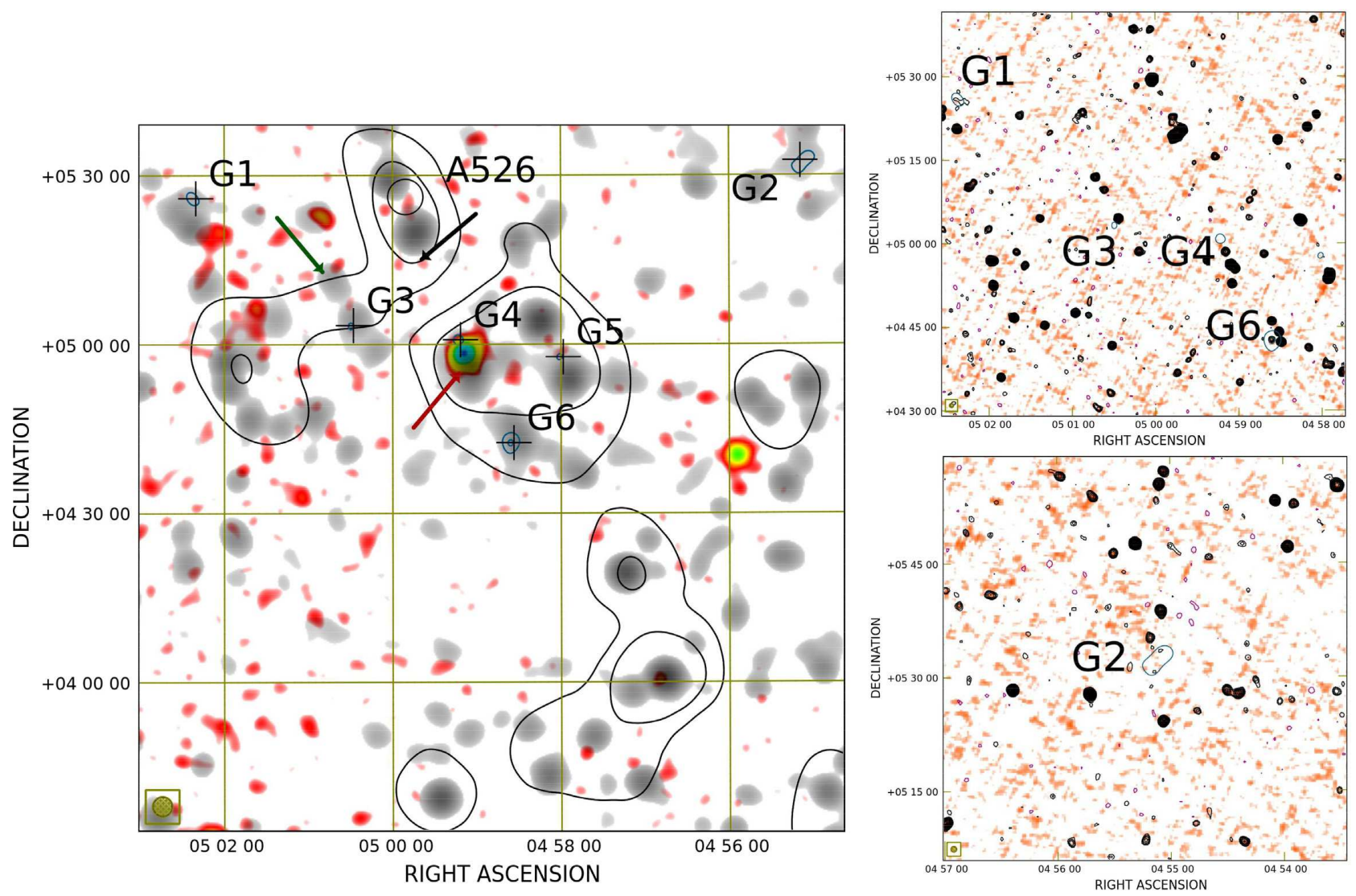

Figure 14. Region G. Left-hand panel: SRT contours $\left(-60,60,150,500 \mathrm{mJy}_{\text {beam }}^{-1}\right.$, negative in magenta and positive in black; resolution 13.9 ar$\mathrm{cmin} \times 12.4$ arcmin) and SRT+NVSS after compact-source subtraction contours (in blue, starting from $3 \sigma$ and the remaining increasing by a factor $\sqrt{2}$; $\sigma=2.5 \mathrm{mJy} \mathrm{beam}^{-1}$, resolution $3.5 \mathrm{arcmin} \times 3.5 \mathrm{arcmin}$ ) overlaid on X-ray emission from the RASS in red colours and radio emission from the SRT+NVSS (resolution 3.5 arcmin) in grey colours. Right-hand panels: NVSS contours $-3 \sigma$ (magenta, $\sigma=0.45 \mathrm{mJy} \mathrm{beam}^{-1}$ ), $3 \sigma$, and increasing by a factor $\sqrt{2}$ (black) overlaid on the TGSS in colours. The black and red arrow mark respectively the locations of the galaxy cluster ZwCl0457.0+0511 and of the compact source PMN J0459+0455, while the green arrow a bridge of radio emission detected by SRT. The colorbars are the same as in Fig. 7.

very faint. They show a radio power comparable to that of radio halos, but fainter X-ray emission $\left(\lesssim 10^{43} \mathrm{erg} \mathrm{s}^{-1}\right)$ and larger size. Their nature is quite mysterious, since they are located in a region of the sky where no galaxy cluster has been identified in the literature.

The radio power versus LLS diagram (right-hand panel of Fig. 18 ) is interesting, as extended diffuse radio sources in clusters are known to follow the correlation

$P_{1.4 \mathrm{GHz}} \propto \operatorname{LLS}^{a}$

between their radio power $P_{1.4 \mathrm{GHz}}$ and their LLS (see e.g. Feretti et al. 2012). The measurements for A520 and A523 sit in the correlation for cluster radio sources, as expected, while the remaining sources are located on the right of this correlation. The source G4 shows a size larger than expected from the correlation observed for radio halos and relics, despite its X-ray luminosity being comparable to that of these sources. The mean power and the mean LLS of the sample (excluding the radio halos in A520 and A523) are respectively $P_{1.4 \mathrm{GHz}}=2.9 \times 10^{24} \mathrm{~W} \mathrm{~Hz}^{-1}$ and LLS $=1.3 \mathrm{Mpc}$. The radio power of the new sources presented in this work correlates with the largest linear size as well. However, this correlation differs from that of radio halos and relics. A linear fit in logarithmic scale of equation (1) gives a slope $a=1.54 \pm 0.03$ for cluster sources and $a=2.04 \pm 0.02$ for the new sources. The values of LLS, $P_{1.4 \mathrm{GHz}}$ and $L_{\mathrm{X}}, 0.1-2.4 \mathrm{keV}$ of the sources presented here have been derived under the assumption that the redshift for these sources is known. As already discussed, the identification of the redshift of these sources is not straightforward and therefore we have adopted the values of the closest radio galaxy or galaxy cluster to the system, or alternatively a redshift $z=0.100$, if no association was possible (see Tables 5 and 6). However, we note that a different redshift would shift the radio power and LLS to lower/higher values but the correlation would remain. In addition, we compare the mean emissivity $\langle J\rangle$ of the new sources to those of the cluster sources, by assuming that they have a spherical symmetry and radius $R=0.5 \times$ LLS. The result is shown in Fig. 19. The histogram reveals two distinct distributions with only a partial overlap. The mean value of the emissivity for cluster sources is $2.7 \times 10^{-41} \mathrm{erg} \mathrm{s}^{-1} \mathrm{~Hz}^{-1} \mathrm{~cm}^{-3}$ $\left(3.0 \times 10^{-42} \mathrm{erg} \mathrm{s}^{-1} \mathrm{~Hz}^{-1} \mathrm{~cm}^{-3}\right.$ when only radio halos are considered), while for the new sources we find a mean value of $3.1 \times$ $10^{-43} \mathrm{erg} \mathrm{s}^{-1} \mathrm{~Hz}^{-1} \mathrm{~cm}^{-3}$. The distribution observed for cluster diffuse radio sources is consistent with the findings of Murgia et al. (2009), who assume a radius given by the e-folding radius of the exponential fit of the radio source brightness profile.

In order to investigate if the new candidate sources are randomly distributed in the sky or instead show a real connection with the filament of galaxy clusters located in the same area (A523, A525, RXC J0503.1+0607, A529, A515, A526, 

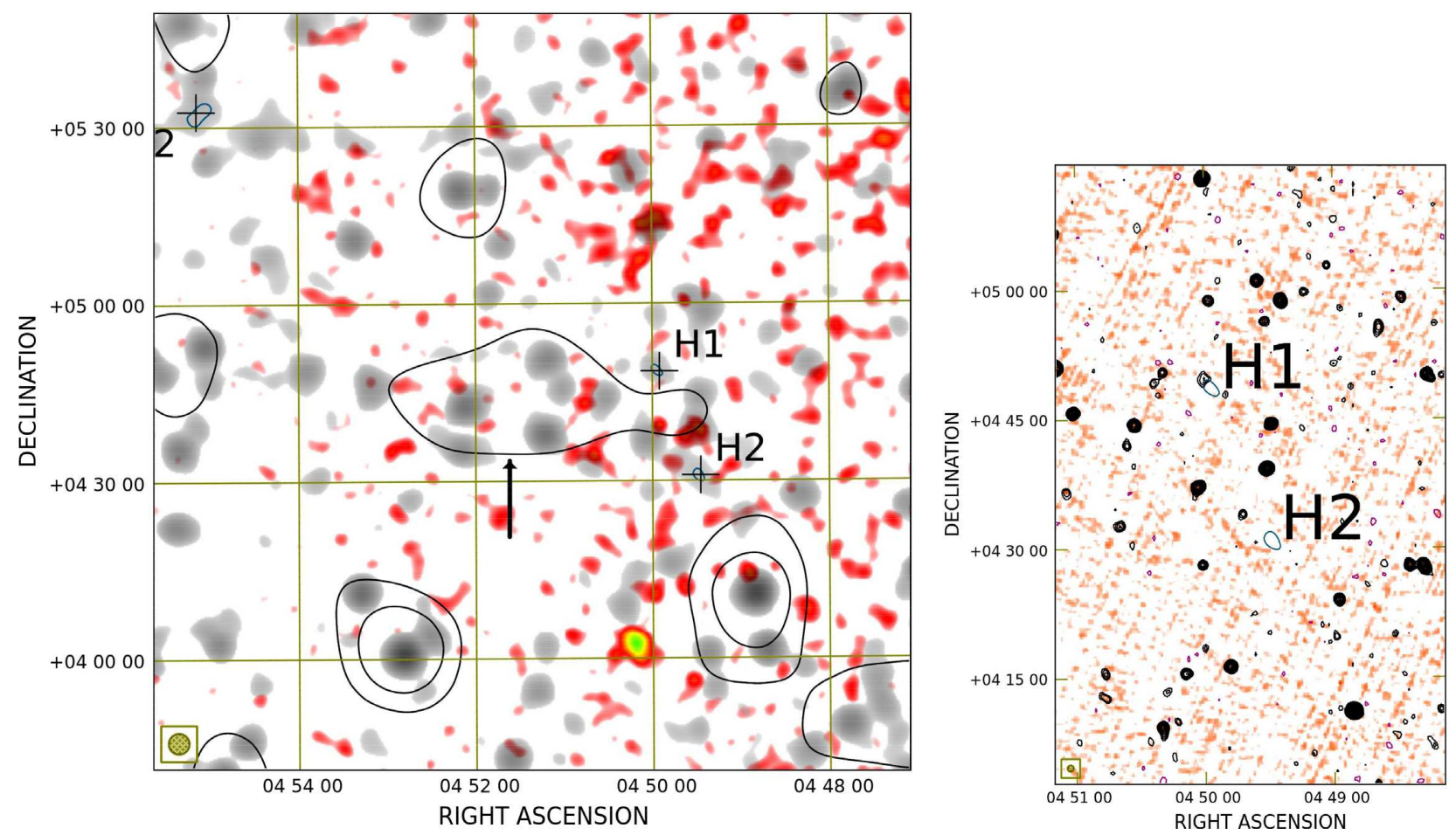

Figure 15. Region H. Left-hand panel: SRT contours $\left(-60,60,150,500 \mathrm{mJy}_{\text {beam }}^{-1}\right.$, negative in magenta and positive in black; resolution 13.9 ar$\mathrm{cmin} \times 12.4 \mathrm{arcmin}$ ) and SRT+NVSS after compact-source subtraction contours (in blue, starting from $3 \sigma$ and the remaining increasing by a factor $\sqrt{2}$; $\sigma=2.5 \mathrm{mJy}_{\text {beam }}{ }^{-1}$, resolution $3.5 \mathrm{arcmin} \times 3.5 \mathrm{arcmin}$ ) overlaid on X-ray emission from the RASS in red colours and radio emission from the SRT+NVSS (resolution 3.5 arcmin) in grey colours. Right-hand panel: NVSS contours $-3 \sigma$ (magenta, $\sigma=0.45 \mathrm{mJy}$ beam $^{-1}$ ), $3 \sigma$, and increasing by a factor $\sqrt{2}$ (black) overlaid on the TGSS in colours. The black arrow marks a blending of point-like sources. The colorbars are the same as in Fig. 7.

RXC J0506.9+0257, A508, and A509), we compute the conditional nearest neighbour distance $D_{\mathrm{CNN}}$ (Okabe \& Miki 1984), defined as

$D_{\mathrm{CNN}}=\frac{1}{N_{\mathrm{s}}+N_{\mathrm{c}}}\left(\sum_{i=1}^{N_{\mathrm{s}}} d_{\mathrm{s} i}+\sum_{j=1}^{N_{\mathrm{c}}} d_{\mathrm{c} j}\right)$

where $N_{\mathrm{s}}$ is the total number of new candidate sources, $N_{\mathrm{c}}$ is the total number of clusters, $d_{\mathrm{s} i}$ is the distance of the $i$-source from the closest cluster, and $d_{\mathrm{cj} j}$ is the distance of the $j$-cluster from the closest new candidate source. We obtain $D_{\mathrm{CNN}}=1.09^{\circ}$

As a comparison we compute $D_{\mathrm{CNN}}$ for about 130000 sets of random new candidate sources, while keeping the cluster coordinates fixed. The coordinates of the random sources have been extracted from uniform distributions in right ascension and declination. In Fig. 20 , we show the distribution of $D_{\mathrm{CNN}}$ for the random sets of sources (black histogram) and for our sample (blue line). The probability of a random association between our new sources and the galaxy cluster filament is less than 2.5 per cent. This means that the number of spurious sources related to noise, or to the Galactic foreground, is small. We verified this point with the help of mock SRT observations that we ran through the same imaging pipeline as the real ones. We find that gain fluctuations are strongly reduced in our images after the application of wavelet and denoising techniques. However, the 18-20 per cent of the diffuse large-scale detected sources could be Galactic. A detailed description of the simulations and of the results is given in Appendix C.

If confirmed, our results reveal a new population of sources, very luminous and extended in radio, but very elusive in X-rays. These sources could be associated with the filaments of the cosmic web. The emission from these structures is believed to be very faint at all wavelengths. Indeed, we are able to detect only a fraction of them in X-rays and with low significance $(1 \sigma)$, while in radio we begin to detect them thanks to the high sensitivity to surface brightness of single-dish observations. The fact that most of our sample is offset to larger sizes (at the same radio power), that the average emissivity is 10-100 smaller than radio halos and relics, and that the X-ray luminosity of the detected sources is about 10-100 weaker than cluster sources, are evidence that we have discovered a new population of sources at lower surface brightness. This is consistent with the thermodynamic properties of the gas in simulated cosmological filaments, whose typical X-ray emissivity is expected to be $\sim 10-100$ smaller than the emissivity of galaxy clusters with the same mass (Gheller et al. 2016).

\subsection{Observations versus simulations}

To interpret our results, we compared the observations with simulations from the sample already introduced in Vazza et al. (2015) and Gheller et al. (2016), obtained with the cosmological grid code ENZO (Bryan et al. 2014). In summary, we used a uniform $1200^{3}$ grid with a resolution of $83 \mathrm{kpc}$ (comoving) to simulate the evolution of a $100 \mathrm{Mpc}^{3}$ region from $z=30$ to $z=0$, assuming a seed magnetic field of $1 \mathrm{nG}$ of primordial origin ${ }^{6}$. The magnetic field in the mag-

${ }^{6} \mathrm{We}$ note that in this work we re-normalize the magnetic field model of Gheller et al. (2016), in which a $0.1 \mathrm{nG}$ initial seed field was assumed. This higher initial field is still within the bound of present constraints derived from the cosmic microwave background (e.g. Subramanian 2016) and it is legitimate because everywhere in the volume our simulated magnetic fields are far from saturation. 

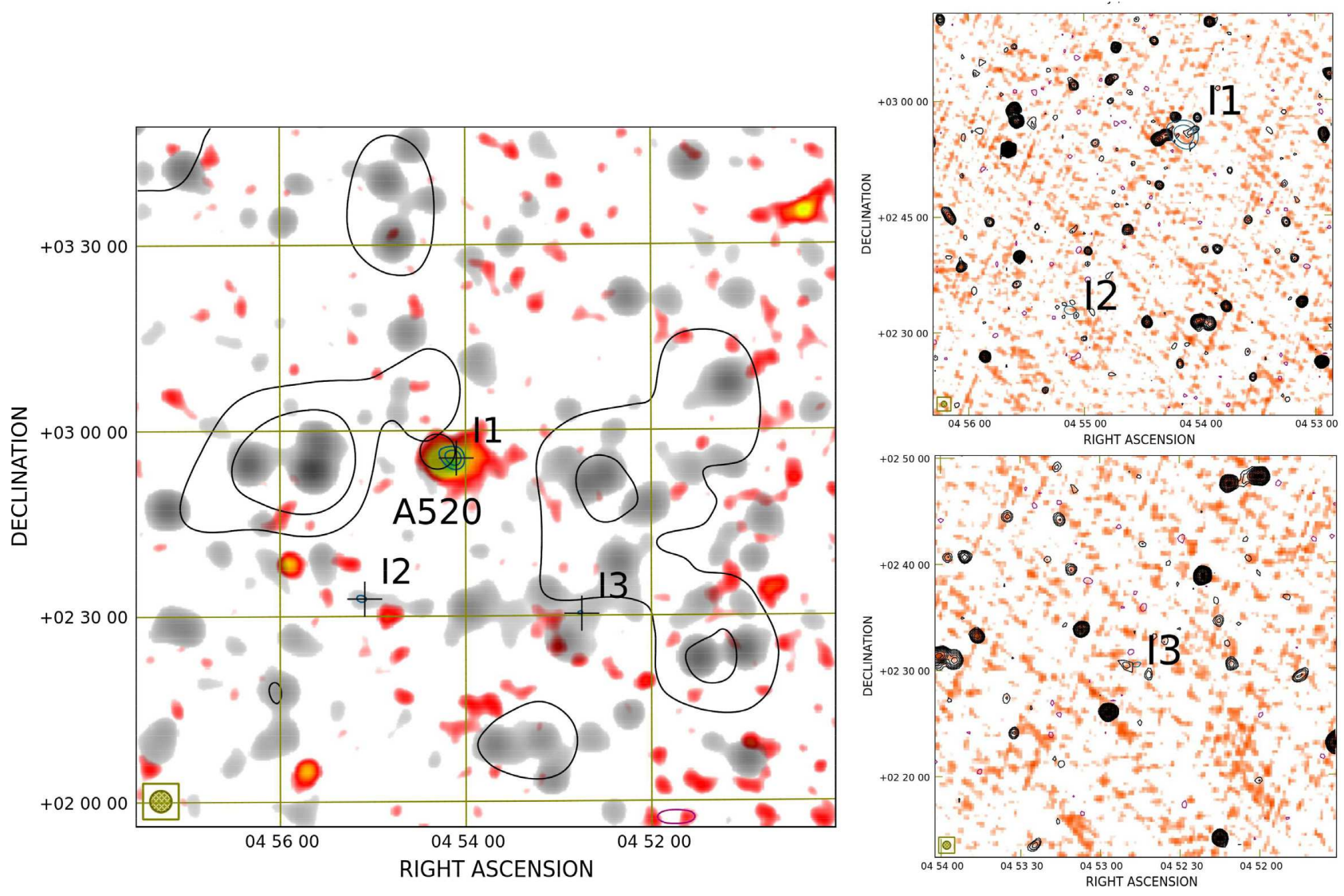

Figure 16. Region I. Left-hand panel: SRT contours $\left(-60,60,150,500 \mathrm{mJy}^{-1}\right.$ beam $^{-1}$, negative in magenta and positive in black; resolution 13.9 ar$\mathrm{cmin} \times 12.4$ arcmin) and SRT+NVSS after compact-source subtraction contours (in blue, starting from $3 \sigma$ and the remaining increasing by a factor $\sqrt{2}$; $\sigma=2.5 \mathrm{mJy}_{\text {beam }}{ }^{-1}$, resolution $\left.3.5 \mathrm{arcmin} \times 3.5 \mathrm{arcmin}\right)$ overlaid on X-ray emission from the RASS in red colours and radio emission from the SRT+NVSS (resolution 3.5 arcmin) in grey colours. Right-hand panels: NVSS contours $-3 \sigma$ (magenta, $\sigma=0.45 \mathrm{mJy} \mathrm{beam}^{-1}$ ), $3 \sigma$, and increasing by a factor $\sqrt{2}$ (black) overlaid on the TGSS in colours. The colorbars are the same as in Fig. 7.

netohydrodynamical (MHD) method uses the conservative Dedner formulation (Dedner et al. 2002) which uses hyperbolic divergence cleaning to keep $\nabla \cdot \vec{B}$ close to zero. Radiative processes and feedback from star-forming regions and/or active galactic nuclei were not included in this run. The assumed cosmology is the $\Lambda \mathrm{CDM}$ cosmological model with density parameters $\Omega_{0}=1.0, \Omega_{\mathrm{BM}}=0.0455$, $\Omega_{\mathrm{DM}}=0.2265$ (BM and DM indicating the baryonic and the dark matter, respectively).

To simulate synchrotron radio emission from the cosmic web, we followed Vazza et al. (2015) and assumed the diffusive shock acceleration of electrons at cosmological shocks, relying on the formalism by Hoeft \& Brüggen (2007) to combine simulated magnetic fields and the distribution of Mach numbers, $\mathcal{M}$, in order to estimate the level of synchrotron emission across the cosmic web, which is computed for each simulated cell as

$$
P_{v} \propto n_{\mathrm{u}} \xi(\mathcal{M}) \cdot M^{3} c_{\mathrm{s}}^{3} S \cdot B^{2}
$$

where $n_{\mathrm{u}}$ is the upstream gas density, $c_{\mathrm{s}}$ is the sound speed in the upstream gas, $B$ is the magnetic field in the shocked cell, and $S$ is the shock surface. The acceleration efficiency of electrons, $\xi(\mathcal{M})$, is taken from Hoeft \& Brüggen (2007) and (in the absence of seed relativistic electrons to re-accelerate) is a steep function of $\mathcal{M}$ for weak shocks, and rapidly saturates to $\xi_{\mathrm{e}} \approx 7 \times 10^{-4}$ for $M>10$ shocks in our model.
Finally, in order to focus on the radio emission produced by filaments in our volume and minimize the contamination by denser structures along the line of sight, we relied on the filament finder presented in Gheller et al. (2016), which allows us to extract the 3D isosurfaces associated with the mild overdensities associated with filaments, as well as to build a catalogue of single filament objects, for which mean thermodynamic and magnetic properties can be computed.

To compare with observation, we produced mock radio observation of the sky model from cosmological simulations, with a procedure similar to Vazza et al. (2015). In particular, we computed the radio emission model at $1.4 \mathrm{GHz}$ (by locating our simulated box at a distance corresponding to $z=0.1$ ) convolving the input sky model with a final beam of 3.5 arcmin $\times 3.5$ arcmin and considering a final sensitivity of $0.05 \mu \mathrm{Jy} \operatorname{arcsec}^{-2}$, the same as those of the image used for our analysis. The result is shown in Fig. 21. Colours in the left-hand panel represent the projected density, while in the right-hand panel, they show the projected full radio emission at the nominal resolution of the simulation $(83 \mathrm{kpc}$ per cell). White contours describe the estimated virial volume of all halos in the box (based on their total matter overdensity). Purple contours show the detectable radio emission in the SRT observing configuration presented in this paper.

Based on the 3D catalogue of filaments obtained in Gheller et al. (2016), we computed the properties of filaments seen in projections in the entire volume, i.e. their total intrinsic radio power at $1.4 \mathrm{GHz}$ 

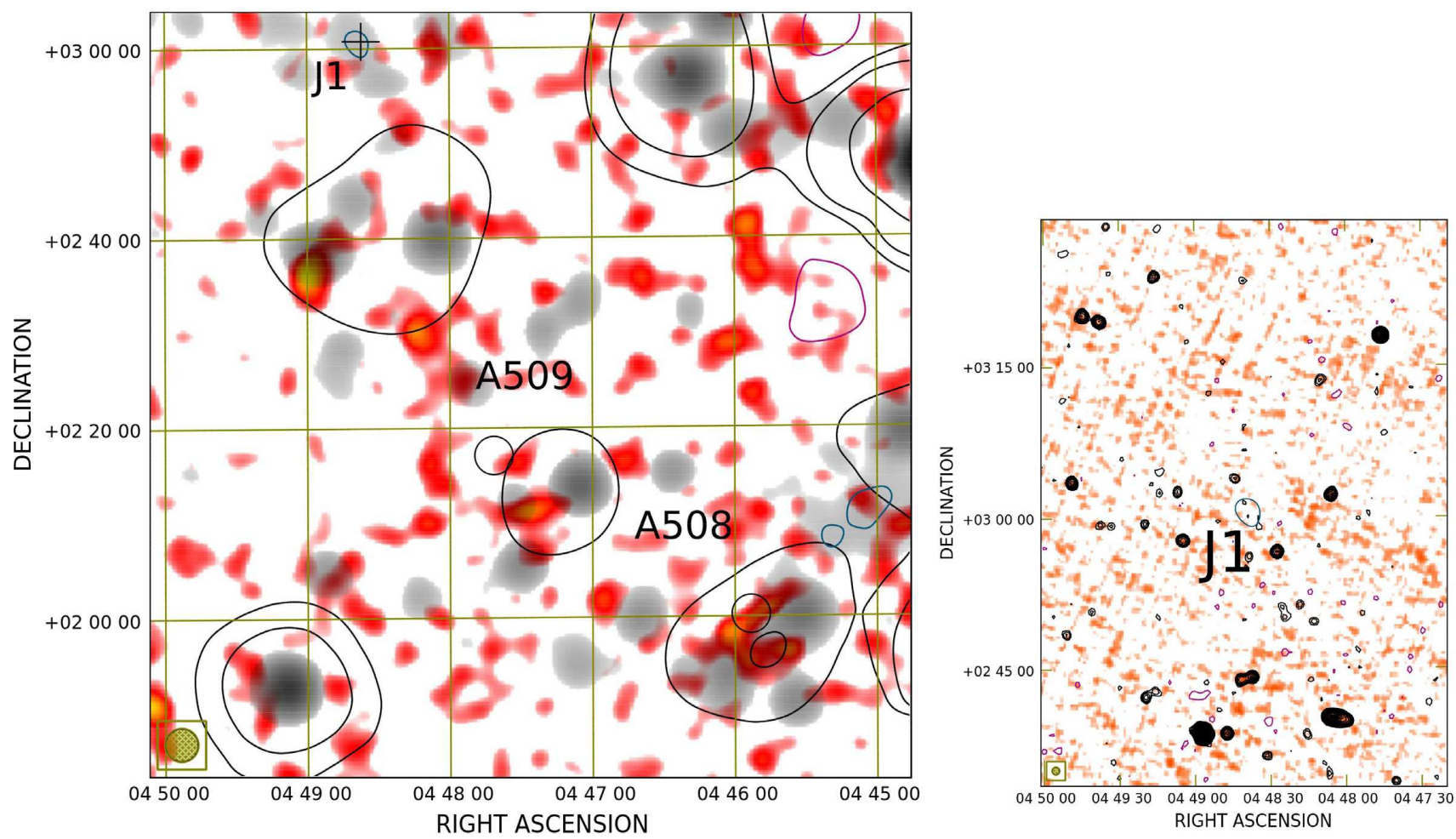

Figure 17. Region J. Left-hand panel: SRT contours $\left(-60,60,150,500 \mathrm{mJy}^{-1}\right.$ beam $^{-1}$, negative in magenta and positive in black; resolution 13.9 ar$\mathrm{cmin} \times 12.4$ arcmin) and SRT+NVSS after compact-source subtraction contours (in blue, starting from $3 \sigma$ and the remaining increasing by a factor $\sqrt{2}$; $\sigma=2.5 \mathrm{mJy}_{\text {beam }}{ }^{-1}$, resolution 3.5 arcmin $\times 3.5$ arcmin) overlaid on X-ray emission from the RASS in red colours and radio emission from the SRT+NVSS (resolution $3.5 \mathrm{arcmin}$ ) in grey colours. Right-hand panel: NVSS contours $-3 \sigma$ (magenta, $\sigma=0.45 \mathrm{mJy} \mathrm{beam}^{-1}$ ), $3 \sigma$, and increasing by a factor $\sqrt{2}$ (black) overlaid on the TGSS in colours. The colorbars are the same as in Fig. 7.
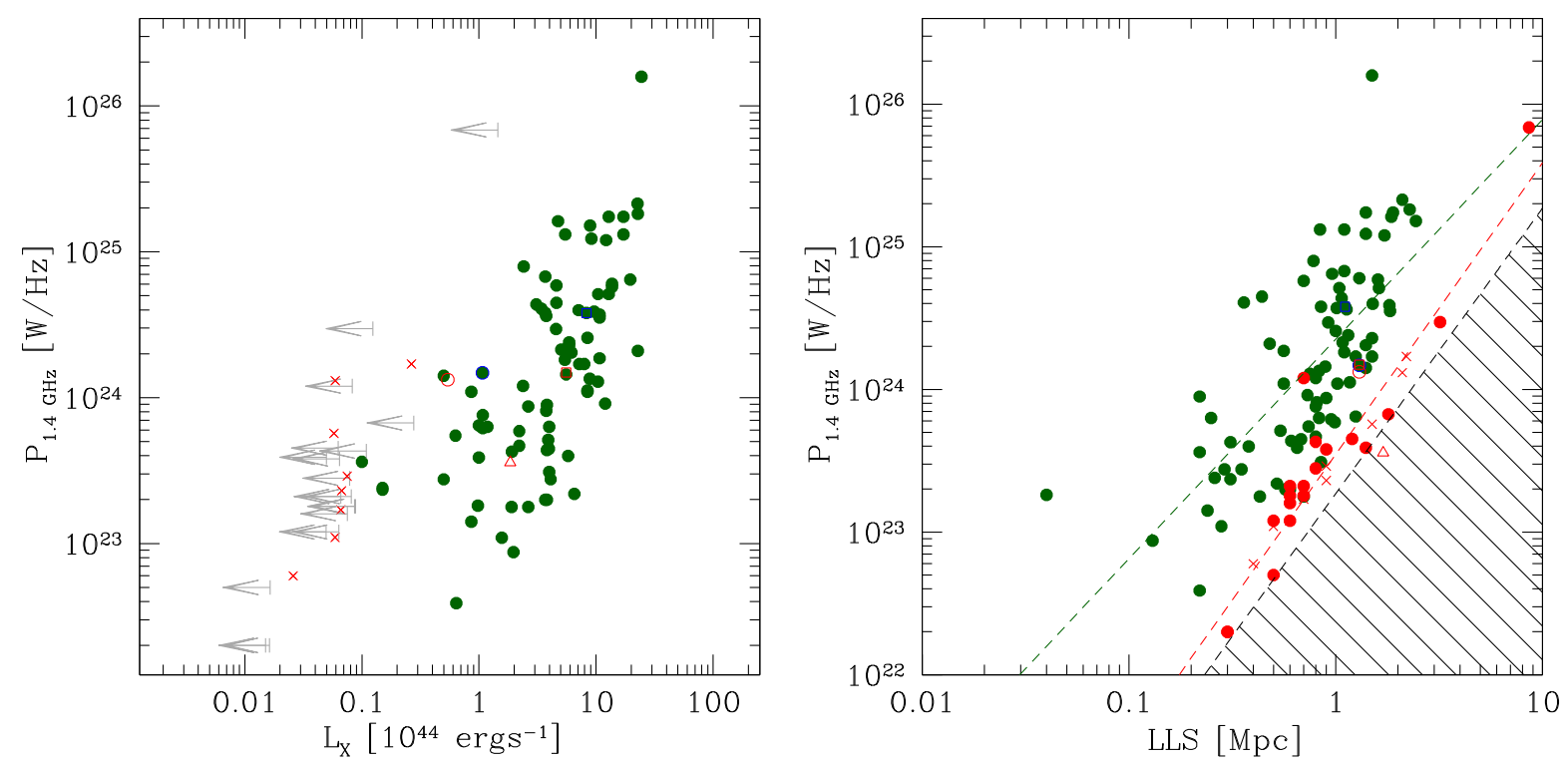

Figure 18. Left-hand panel: Radio power at $1.4 \mathrm{GHz} P_{1.4 \mathrm{GHz}}$ versus X-ray luminosity in the energy band $0.1-2.4 \mathrm{keV} L_{\mathrm{X}}, 0.1-2.4 \mathrm{keV}$. Right-hand panel: Radio power $P_{1.4 \mathrm{GHz}}$ versus LLS both at $1.4 \mathrm{GHz}$. Green dots and blue open symbols are from Feretti et al. (2012). Red symbols are measurements from this work: open symbols identify A523 (circle), A520 (square), G4 (triangle), while crosses identify the remaining sources with an X-ray identification. Grey arrows are upper limits from this work. The shaded area in the right-hand panel indicates the region of the $\left(P_{1.4 \mathrm{GHz}}, \mathrm{LLS}\right)$ plane that cannot be accessed considering the $2 \sigma\left(\sigma=2.5 \mathrm{mJy}_{\text {beam }}{ }^{-1}\right)$ sensitivity of the SRT+NVSS image after point-source subtraction. Dashed lines represent a linear fit in logarithmic scale of equation (1) for cluster sources (green) and for the new sources (red). 


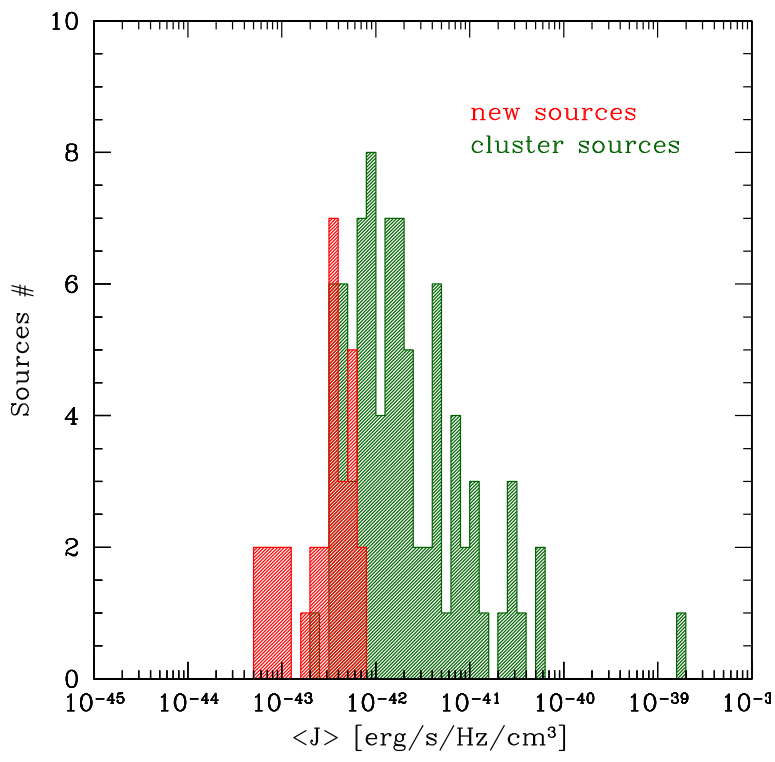

Figure 19. Histogram in logarithmic scale of the mean emissivity of the new sources presented in this paper (red) compared to the cluster diffuse radio sources from Feretti et al. (2012) (green).

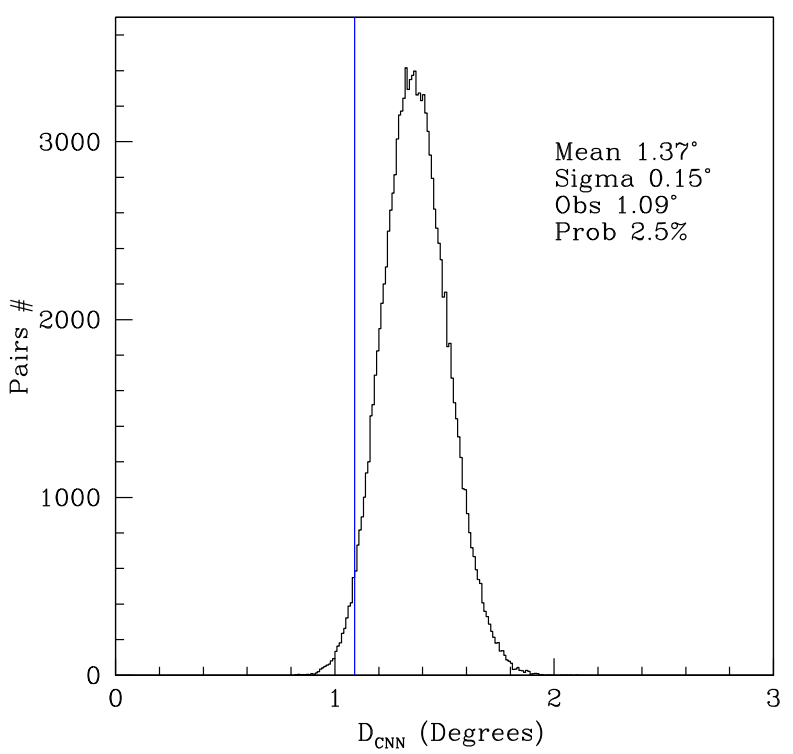

Figure 20. Distribution of $D_{\mathrm{CNN}}$ for a random sets of sources (black histogram). The histogram is well described by a Gaussian distribution with mean $1.37^{\circ}$ and standard deviation $0.15^{\circ}$. The $D_{\mathrm{CNN}}$ for our sample is shown by the blue line.

(prior to any observational cut) and their largest linear size. By considering the noise and resolution of the SRT observations, only a very tiny fraction $\left(\leq 10^{-4}\right)$ of the radio emitting surface of the cosmic web in the simulation survives. We recomputed the corresponding radio power and LLS of these objects. In Fig. 22, we show the radio power at $1.4 \mathrm{GHz}$ versus the LLS for all the simulated objects before the SRT observing parameters are considered (grey crosses), for simulated objects after the SRT noise and spatial resolution are applied (empty black dots), and for observed objects (full red dots). The crosses represent all the diffuse large-scale sources in the image before any observing parameter (noise and convolution) is applied. After the SRT noise and spatial resolution are taken into account, the detected sources are only those marked by empty black circles. The few detectable emission patches populate almost the same region of the (LLS, $P_{1.4 \mathrm{GHz}}$ ) plane as the observed candidate sources presented in this paper. In most cases, as suggested by the map in Fig. 21, these large diffuse emission regions are associated with very peripheral shocks at the crossroad between the outer virial volume of massive galaxy clusters and the filaments connecting them, which are typically detectable only in very crowded overdense regions, similar to the high-density field observed in this work.

In Fig. 23, we show the mean magnetic field $\langle B\rangle$ and mean temperature $\langle T\rangle$ versus $P_{1.4 \mathrm{GHz}}$ (top and middle panels), and $\langle T\rangle$ versus $\langle B\rangle$ (bottom panel), for the simulated filaments that host emission patches which should be detectable by our SRT observation. The average properties of the host objects are typical of the most massive filaments in our simulations (Gheller et al. 2016), with $n /\langle n\rangle \sim 50-100,\langle B\rangle \sim 10-50 \mathrm{nG}, T \sim 2 \times 10^{5}-5 \times 10^{6} \mathrm{~K}$, yet the detectable regions only cover a tiny fraction (a few per cent) of the filaments' projected area, and they tend to be associated with the densest and hottest portions of filaments, connecting to the surrounding clusters. With the assumed prescription for extragalactic magnetic fields and electron acceleration at shocks, the regions which are within the range of detection in our SRT observation typically have an average magnetic field along the line of sight of $\sim 0.02-0.05 \mu \mathrm{G}$.

\section{SUMMARY AND CONCLUSIONS}

In this work, we report the detection of diffuse radio emission which might be associated with a large-scale filament of the cosmic web covering a $8^{\circ} \times 8^{\circ}$ area in the sky, likely associated with a $z$ $\approx 0.1$ overdensity traced by nine massive galaxy clusters. To investigate the presence of large-scale diffuse synchrotron emission beyond the cluster periphery, we observed this area with the Sardinia Radio Telescope. These low spatial resolution data have been combined with high-resolution observations from the NRAO Very Large Array Sky Survey, to permit separation of the diffuse largescale synchrotron emission from that of embedded discrete radio sources.

By inspecting the field of view, we identified 35 patches of diffuse emission with significance above $3 \sigma$. Two are the cluster sources already known in the direction of the galaxy clusters A523 (source A1) and A520 (source I1), five sources (C1, C6, C9, E2, and G6) are probably the leftover of the compact-source subtraction process or artefacts, and the remaining 28 sources represent diffuse synchrotron radio emission with no obvious interpretation. To shed light on the nature of these new sources, we studied their radio and X-ray properties. Only eleven sources have an X-ray count rate significantly above $1 \sigma$. These sources are A1 (diffuse emission in A523), A2, A3, C3, C8, E3, G3, G4, G5, I1 (diffuse emission in A520), and I3. Apart from the two radio halos in A520 and A523, one of the significant X-ray sources (G4) sits in the $L_{\mathrm{X}}, 0.1-2.4 \mathrm{keV}-$ $P_{1.4 \mathrm{GHz}}$ correlation observed for radio halos and relics but has a larger size than expected for cluster sources with this power. The remaining eight sources show an X-ray luminosity between 10 and 100 times lower than expected from their radio power given the correlation observed for cluster sources, with an average X-ray luminosity of $L_{\mathrm{X}, 0.1-2.4 \mathrm{keV}}=8.4 \times 10 \mathrm{erg} \mathrm{s}^{-1}$. They populate a new region of the $\left(L_{\mathrm{X}}, 0.1-2.4 \mathrm{keV}, P_{1.4 \mathrm{GHz}}\right)$ plane that was previously unsampled. 

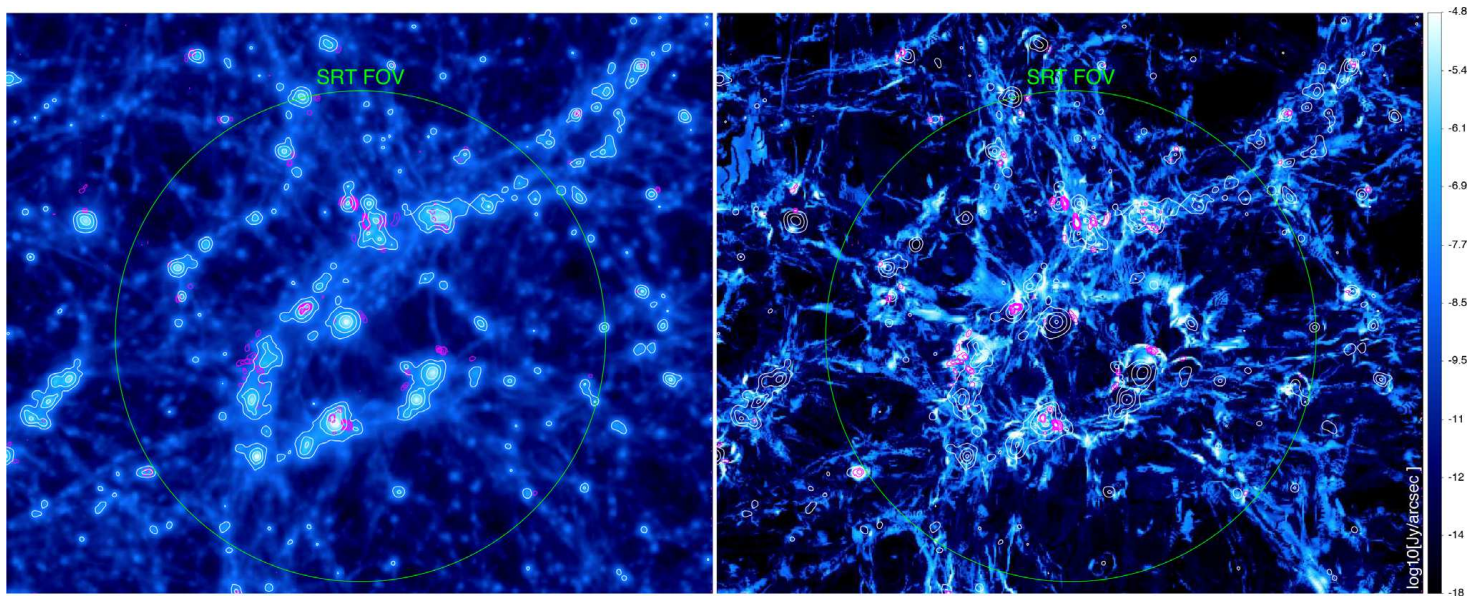

Figure 21. Left-hand panel: Estimated virial volume of all halos in the box (based on their total matter density, white contours) and detectable radio emission in the SRT observing configuration presented in this paper (purple contours) overlaid on the projected gas density (colours). Right-hand panel: The colours show the projected full radio emission at the nominal resolution of the simulation ( $83 \mathrm{kpc}$ per cell) and assuming this field is at $z=0.1$. The contours are the same as in the left-hand panel. The green circles in both images represent the field of view of SRT if the simulated volume is located at $z=0.1$.

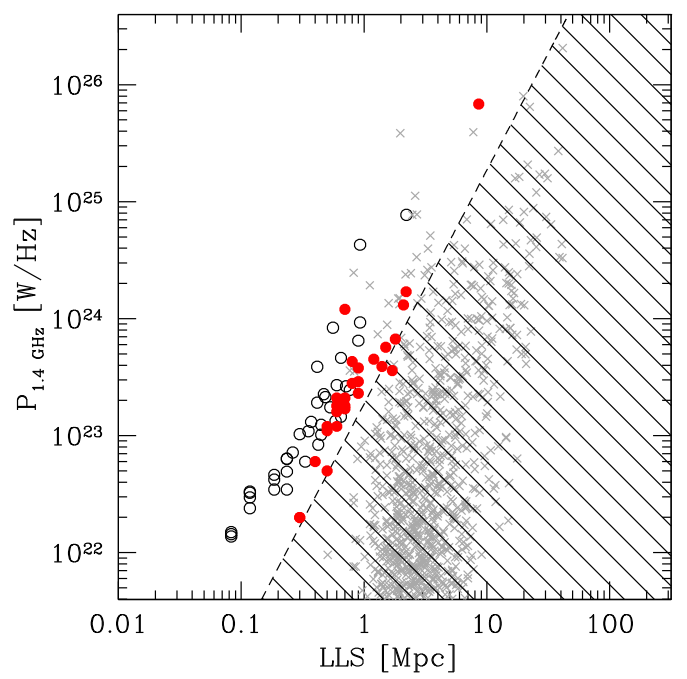

Figure 22. Radio power at $1.4 \mathrm{GHz}$ versus the LLS at $1.4 \mathrm{GHz}$ for the all simulated objects before the SRT observing parameters are considered (grey crosses), for simulated objects after the SRT noise and spatial resolution are applied (empty black dots), and for observed objects (full red dots). The shaded area indicates the region of the $\left(P_{1.4 \mathrm{GHz}}, \mathrm{LLS}\right)$ plane that cannot be accessed considering the $2 \sigma\left(\sigma=2.5 \mathrm{mJy}\right.$ beam $\left.^{-1}\right)$ sensitivity of the SRT+NVSS image after point-source subtraction.

Some of these are particularly interesting. Source A2 in combination with the source south-west of it and with Source A1 (the radio halo in A523) could represent the first case of a triple radio halo, particularly interesting because of its association with an underluminous X-ray system. Source E1 is the largest and the more powerful diffuse radio source in the field. Its size is 25 arcmin and its location on the plane of the sky is in the same direction as the filament connecting A523-A525 to A509-A508. Alternatively, it could be located at higher redshift, if associated with $4 \mathrm{C}+06.21$, the source embedded in it. Whether this source belongs to the filament or it is instead at higher redshift has an impact on its size that ranges between 3 and $8.7 \mathrm{Mpc}$, and in the latter case it would rep- resent the largest extragalactic diffuse synchrotron source known to date.

Overall, these objects have distinct properties from known radio halos and relics. Indeed, they show lower X-ray luminosity in the $0.1-2.4 \mathrm{keV}$ band and a larger LLS than expected for a given radio power from the correlation observed for cluster sources. The mean power and the mean LLS of the sample (excluding the radio halos in A520 and in A523) are respectively $P_{1.4 \mathrm{GHz}}=2.9 \times 10^{24} \mathrm{~W} \mathrm{~Hz}^{-1}$ and LLS $=1.3 \mathrm{Mpc}$. The average radio emissivity and the X-ray luminosity of these sources are about 10-100 weaker than those of cluster diffuse sources. These characteristics are indicative of a very faint new population of sources. The conditional nearest neighbour distance of clusters and candidate new sources indicates that the probability of a random association between our new sources and the galaxy cluster filament is less than 2.5 per cent. This means that it is unlikely spurious sources related to noise or to the Galactic foreground are present. A comparison between simulations and observations shows that the radio powers and radio sizes of these sources are comparable to those expected by the brightest patches of diffuse synchrotron radio emission associated with the filaments of the cosmic web and correspond to an average magnetic field along the line of sight of $\sim 0.02-0.05 \mu \mathrm{G}$.

High-spatial resolution and deep radio, X-ray and optical followups are essential to shed light on the nature of these puzzling sources.

\section{ACKNOWLEDGEMENTS}

We thank the anonymous referee for the useful comments and suggestions that helped to improve the paper. The Sardinia Radio Telescope (Bolli et al. 2015; Prandoni et al. 2017) is funded by the Department of University and Research (MIUR), Italian Space Agency (ASI), and the Autonomous Region of Sardinia (RAS) and is operated as National Facility by the National Institute for Astrophysics (INAF). The development of the SARDARA back-end was funded by the Autonomous Region of Sardinia (RAS) using resources from the Regional Law 7/2007 'Promotion of the scientific research and technological innovation in Sardinia' in the context of the research project CRP-49231 (year 2011, PI Andrea Possenti): 'High-resolution sampling of the Universe in the radio band: an 

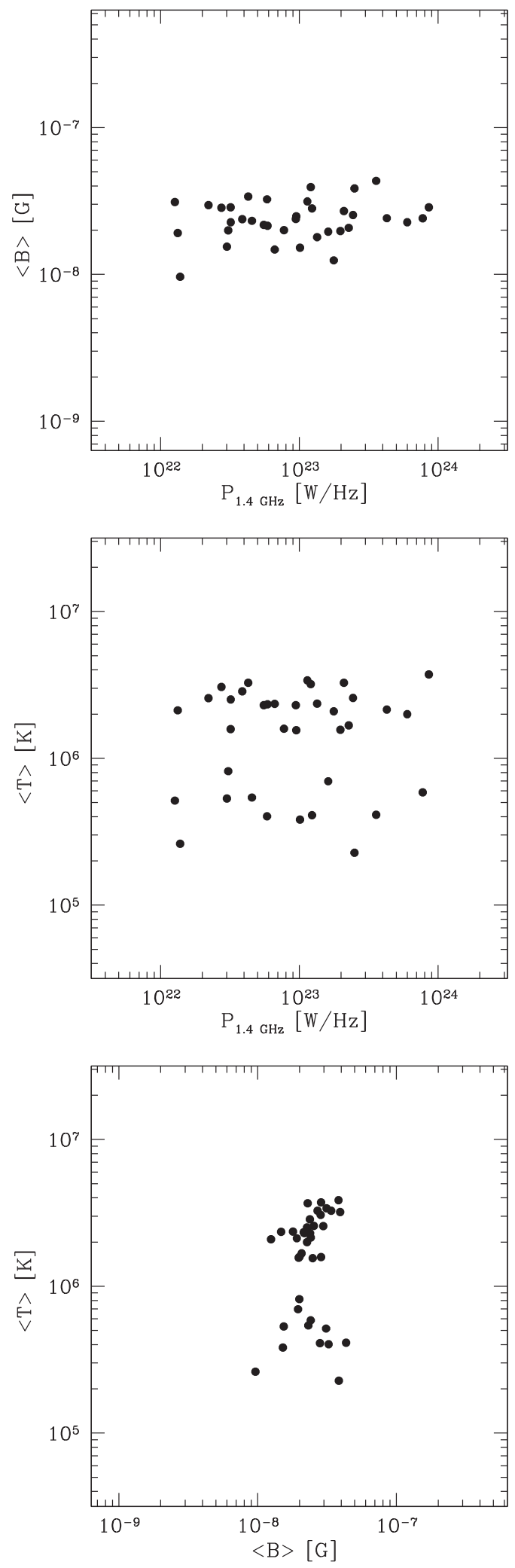

Figure 23. Top panel: Mean volume-weighted magnetic field $\langle B\rangle$ versus $P_{1.4 \mathrm{GHz}}$. Middle panel: Mean temperature $\langle T\rangle$ versus $P_{1.4 \mathrm{GHz}}$. Bottom panel: $\langle T\rangle$ versus $\langle B\rangle$. unprecedented instrument to understand the fundamental laws of the nature'. The National Radio Astronomy Observatory (NRAO) is a facility of the National Science Foundation, operated under cooperative agreement by Associated Universities, Inc. This research made use of the NASA/IPAC Extragalactic Database (NED) which is operated by the Jet Propulsion Laboratory, California Institute of Technology, under contract with the National Aeronautics and Space Administration. VV acknowledges Marta Burgay for providing computational resources. FL gratefully acknowledges Sardinia Regional Government for the financial support of her PhD scholarship (P.O.R. Sardegna F.S.E. Operational Programme of the Autonomous Region of Sardinia, European Social Fund 2007-2013 Axis IV Human Resources, Objective 1.3, Line of Activity 1.3.1.). Basic research in radio astronomy at the Naval Research Laboratory is funded by 6.1 Base funding. FV acknowledges the usage of computational resources at CSCS (ETHZ in Lugano) under allocations s701 and the financial support from the ERC Starting Grant 'MAGCOW', no. 714196. SC research is supported by the South African Research Chairs Initiative of the Department of Science and Technology and National Research Foundation of South Africa (Grant No. 77948).

\section{REFERENCES}

Baars J. W. M., Genzel R., Pauliny-Toth I. I. K., Witzel A., 1977, A\&A, 61, 99

Bagchi J., Enßlin T. A., Miniati F., Stalin C. S., Singh M., Raychaudhury S., Humeshkar N. B., 2002, New Astron., 7, 249

Baiesi-Pillastrini G. C., Palumbo G. G. C., Vettolani G., 1984, A\&AS, 56, 363

Böhringer H. et al., 2000, ApJS, 129, 435

Bolli P. et al., 2015, J. Astron. Instrum., 4, 1550008-880

Bonafede A. et al., 2009, A\&A, 503, 707

Boschin W., Girardi M., Barrena R., 2013, MNRAS, 434, 772

Brown S. et al., 2017, MNRAS, 468, 4246

Brüggen M., Ruszkowski M., Simionescu A., Hoeft M., Dalla Vecchia C., 2005, ApJ, 631, L21

Bryan G. L. et al., 2014, ApJS, 211, 19

Carretti E. et al., 2013, MNRAS, 430, 1414

Cavagnolo K. W., Donahue M., Voit G. M., Sun M., 2008, ApJ, 682, 821

Cen R., Ostriker J. P., 1999, ApJ, 514, 1

Chow-Martínez M., Andernach H., Caretta C. A., Trejo-Alonso J. J., 2014, MNRAS, 445, 4073

Colberg J. M., Krughoff K. S., Connolly A. J., 2005, MNRAS, 359, 272

Condon J. J. et al., 1998, AJ, 115, 1693

Crawford C. S. et al., 1995, MNRAS, 274, 75

Davé R. et al., 2001, ApJ, 552, 473

de Graaff A., Cai Y.-C., Heymans C., Peacock J. A., 2017, preprint (arXiv: 1709.10378)

Dedner A., Kemm F., Kröner D., Munz C.-D., Schnitzer T., Wesenberg M., 2002, J. Comput. Phys., 175, 645

Drinkwater M. J. et al., 1997, MNRAS, 284, 85

Eckert D. et al., 2015, Nature, 528, 105

Einasto M., Einasto J., Tago E., Müller V., Andernach H., 2001, AJ, 122, 2222

Farnsworth D., Rudnick L., Brown S., Brunetti G., 2013, ApJ, 779, 189

Fatigoni S., 2017, Master thesis . Università La Sapienza, Rome, Italy

Feretti L., Giovannini G., Govoni F., Murgia M., 2012, A\&AR, 20, 54

Finoguenov A. et al., 2007, ApJS, 172, 182

Gheller C., Vazza F., Brüggen M., Alpaslan M., Holwerda B. W., Hopkins A. M., Liske J., 2016, MNRAS, 462, 448

Giovannini G., Tordi M., Feretti L., 1999, New Astron., 4, 141

Giovannini G., Bonafede A., Feretti L., Govoni F., Murgia M., 2010, A\&A, 511, L5 
Giovannini G., Feretti L., Girardi M., Govoni F., Murgia M., Vacca V., Bagchi J., 2011, A\&A, 530, L5

Giovannini G., Vacca V., Girardi M., Feretti L., Govoni F., Murgia M., 2013, MNRAS, 435, 518

Girardi M., Barrena R., Boschin W., Ellingson E., 2008, A\&A, 491, 379

Girardi M. et al., 2016, MNRAS, 456, 2829

Govoni F., Feretti L., Giovannini G., Böhringer H., Reiprich T. H., Murgia M., 2001, A\&A, 376, 803

Govoni F., Markevitch M., Vikhlinin A., van Speybroeck L., Feretti L., Giovannini G., 2004, ApJ, 605, 695

Govoni F., Murgia M., Feretti L., Giovannini G., Dallacasa D., Taylor G. B., 2005, A\&A, 430, L5

Govoni F., Murgia M., Feretti L., Giovannini G., Dolag K., Taylor G. B., 2006, A\&A, 460, 425

Govoni F. et al., 2017, A\&A, 603, A122

Gregory P. C., Scott W. K., Douglas K., Condon J. J., 1996, ApJS, 103, 427

Haines C. P. et al., 2017, MNRAS, 477, 4931

Hoeft M., Brüggen M., 2007, MNRAS, 375, 77

Hurier G., Macías-Pérez J. F., Hildebrandt S., 2013, A\&A, 558, A118

Intema H. T., Jagannathan P., Mooley K. P., Frail D. A., 2017, A\&A, 598, A78

Kronberg P. P., Kothes R., Salter C. J., Perillat P., 2007, ApJ, 659, 267

Lane W. M., Cotton W. D., van Velzen S., Clarke T. E., Kassim N. E., Helmboldt J. F., Lazio T. J. W., Cohen A. S., 2014, MNRAS, 440, 327

Loi F. et al., 2017, MNRAS, 472, 3605

Macario G. et al., 2014, A\&A, 565, A13

Mahdavi A., Hoekstra H., Babul A., Balam D. D., Capak P. L., 2007, ApJ, 668,806

Mann A. W., Ebeling H., 2012, MNRAS, 420, 2120

Markevitch M., Govoni F., Brunetti G., Jerius D., 2005, ApJ, 627, 733

Melis A. et al., 2018, J. Astronom. Instrum., 7, 1850004

Murgia M., Govoni F., Feretti L., Giovannini G., Dallacasa D., Fanti R., Taylor G. B., Dolag K., 2004, A\&A, 424, 429

Murgia M. et al., 2009, A\&A, 499, 679

Murgia M., Govoni F., Feretti L., Giovannini G., 2010, A\&A, 509, A86

Murgia M. et al., 2016, MNRAS, 461, 3516

Okabe A., Miki F., 1984, Environ. Plan. A, 16, 163

Orrù E., Murgia M., Feretti L., Govoni F., Brunetti G., Giovannini G., Girardi M., Setti G., 2007, A\&A, 467, 943

Ostrander E. J., Nichol R. C., Ratnatunga K. U., Griffiths R. E., 1998, AJ, 116,2644

Perley R. A., Butler B. J., 2013, ApJS, 204, 19

Pizzo R. F., de Bruyn A. G., Feretti L., Govoni F., 2008, A\&A, 481, L91

Planck Collaboration VIII, 2013, A\&A, 550, A134

Planck Collaboration XVI, 2014, A\&A, 571, A16

Planck Collaboration XXII, 2016a, A\&A, 594, A22

Planck Collaboration XXVII, 2016b, A\&A, 594, A27

Prandoni I. et al., A\&A, 2017, A40, 608

Reich W., 1982, A\&AS, 48, 219

Rines K., Geller M. J., Kurtz M. J., Diaferio A., 2003, AJ, 126, 2152

Roger R. S., Costain C. H., Bridle A. H., 1973, AJ, 78, 1030

Ryu D., Kang H., Cho J., Das S., 2008, Science, 320, 909

Scaife A. M. M., Heald G. H., 2012, MNRAS, 423, L30

Struble M. F., Rood H. J., 1999, ApJS, 125, 35

Subramanian K., 2016, Rep. Prog. Phys., 79, 076901

Vacca V., Murgia M., Govoni F., Feretti L., Giovannini G., Orrù E., Bonafede A., 2010, A\&A, 514, A71

Vacca V., Feretti L., Giovannini G., Govoni F., Murgia M., Perley R. A., Clarke T. E., 2014, A\&A, 561, A52

Valente G. et al., 2010 , in Proc. SPIE Conf. Ser. Vol. 7741. SPIE, Bellingham, p. 774126

van Weeren R. J. et al., 2013, ApJ, 769, 101

Van Weeren R. J. et al., 2016, American Astronomical Society Meeting Abstracts, 227, 202.09

Vazza F., Brüggen M., Gheller C., Wang P., 2014, MNRAS, 445, 3706

Vazza F., Ferrari C., Brüggen M., Bonafede A., Gheller C., Wang P., 2015, A\&A, 580, A119
Vernstrom T., Gaensler B. M., Brown S., Lenc E., Norris R. P., 2017, MNRAS, 467, 4914

Veron-Cetty M.-P., Veron P., 1996, ESO Scientific Report, 7th edn. European Southern Observatory (ESO), Garching

Voges W. et al., 1999, A\&A, 349, 389

Xu J., Han J. L., 2014, MNRAS, 442, 3329

Zwicky F., Karpowicz M., Kowal C. T., 1965, in CGCG5, Vol. V, Catalogue of Galaxies and of Clusters of Galaxies. California Institute of Technology, Pasadena

\section{APPENDIX A: COMPACT SOURCES IN THE FIELD}

This field of view is rich in radio sources, with a total number of thousands at $1.4 \mathrm{GHz}$. To further cross-check the flux density scale of our data, we measured the flux density of a sample of sources that satisfies the following requirements: peak brightness at $1.4 \mathrm{GHz}$ larger than $200 \mathrm{mJy}$ and flux larger than the $3 \sigma$ simultaneously at all frequencies (with $\sigma$ being the uncertainty in the flux measurement at the corresponding frequency). For the sake of comparison, we estimated the flux and its uncertainty over the frequency range $74 \mathrm{MHz}-5 \mathrm{GHz}$, by using the following observations after convolving all the images at the resolution of the GB6 (3.6 $\operatorname{arcmin} \times 3.4 \operatorname{arcmin})$ :

(i) the VLA Low-Frequency Sky Survey Redux (VLSSr, Lane et al. 2014) at $74 \mathrm{MHz}\left(\sigma_{\mathrm{VLSSr}}=300 \mathrm{mJy}\right)$;

(ii) the TIFR GMRT Sky Survey Alternative Data Release (TGSS ADR, Intema et al. 2017) at $150 \mathrm{MHz}\left(\sigma_{\mathrm{TGSS}}=50 \mathrm{mJy}\right)$;

(iii) the NVSS image, at $1.4 \mathrm{GHz}\left(\sigma_{\mathrm{NVSS}}=2 \mathrm{mJy}\right)$;

(iv) the combined SRT and NVSS image, at $1.4 \mathrm{GHz}$ $(\sigma$ SRT + NVSS $=3 \mathrm{mJy})$;

(v) the Green Bank 6-cm survey (GB6, Gregory et al. 1996) at $4.85 \mathrm{GHz}\left(\sigma_{\mathrm{GB} 6}=5 \mathrm{mJy}\right)$.

All these data are in the Baars et al. (1977) flux scale, apart from the TGSS ADR and the VLLSr that have been calibrated according to respectively the Scaife \& Heald (2012) and the Roger, Costain \& Bridle (1973) flux density scale. Therefore, we corrected the TGSS and VLSSr fluxes accordingly. Concerning the VLSSr, we additionally increased the uncertainty in the flux measurement, as suggested by Lane et al. (2014). The flux has been evaluated by applying a cut in brightness in the NVSS image equal to $5 \sigma_{\mathrm{NVSS}}$ and by masking all the other images accordingly. This translates to a total of 24 sources. In Table A1, we report the source ID, the RA, and Dec. coordinates (J2000) of the peak flux in the combined SRT+NVSS image, and the peak flux as measured from the VLSSr, TGSS, NVSS, combined SRT+NVSS, GB6, along with their uncertainties that have been computed by adding in quadrature the statistic and systematic (3 per cent of the flux) uncertainties. The flux measurements from the NVSS and the SRT+NVSS combined images have been compared to cross-check the flux scale after the combination of the data. In Fig. A1, we show the flux of the SRT+NVSS image versus the NVSS flux (dots) along with a linear fit (dashed line). The slope of the fit is 0.97 , revealing a good agreement of the two flux measurements within the errors.

To compare the fluxes at $1.4 \mathrm{GHz}$ with the measurements at the other radio frequencies, we fit the fluxes from VLSSr, TGSS, SRT+NVSS, and GB6 versus frequency with a power law

$S_{v} \propto v^{-\alpha}$,

where $v$ is the observing frequency and $\alpha$ is the spectral index. During the fitting procedure the relative errors were increased to 
Table A1. Radio sources with peak brightness at $1.4 \mathrm{GHz}$ larger than $200 \mathrm{mJy}$.

\begin{tabular}{|c|c|c|c|c|c|c|c|c|c|}
\hline \# & $\begin{array}{l}\text { RA (J2000) } \\
\text { h:m:s }\end{array}$ & $\begin{array}{c}\text { Dec. (J2000) } \\
\circ:^{\prime \prime}{ }^{\prime \prime}\end{array}$ & $\begin{array}{c}S_{\mathrm{VLSSr}} \\
\mathrm{Jy}\end{array}$ & $\begin{array}{c}S_{\mathrm{TGSS}} \\
\text { Jy }\end{array}$ & $\begin{array}{c}S_{\mathrm{NVSS}} \\
\mathrm{Jy}\end{array}$ & $\begin{array}{c}S_{\mathrm{SRT}+\mathrm{NVSS}} \\
\mathrm{Jy}\end{array}$ & $\begin{array}{l}S_{\mathrm{GB} 6} \\
\mathrm{mJy}\end{array}$ & $\alpha$ & $\chi_{\text {red }}^{2}$ \\
\hline 1 & $5: 11: 37$ & $9: 31: 21.40$ & $6.8 \pm 0.6$ & $4.3 \pm 0.2$ & $0.39 \pm 0.01$ & $0.33 \pm 0.01$ & $81 \pm 10$ & $1.07_{-0.05}^{0.05}$ & 2.42 \\
\hline 3 & $5: 04: 31$ & $8: 27: 00.24$ & $2.4 \pm 0.7$ & $2.3 \pm 0.1$ & $0.34 \pm 0.01$ & $0.32 \pm 0.01$ & $85 \pm 12$ & $0.87_{-0.06}^{0.06}$ & 3.89 \\
\hline 4 & $5: 01: 58$ & $6: 50: 57.71$ & $2.6 \pm 0.6$ & $3.3 \pm 0.1$ & $0.42 \pm 0.01$ & $0.42 \pm 0.01$ & $97 \pm 10$ & $0.87_{-0.05}^{0.05}$ & 12.17 \\
\hline 5 & 5:03:03 & $6: 09: 58.82$ & $2.7 \pm 0.7$ & $3.1 \pm 0.2$ & $0.54 \pm 0.02$ & $0.55 \pm 0.02$ & $211 \pm 14$ & $0.70_{-0.05}^{0.05}$ & 3.97 \\
\hline 7 & $5: 06: 10$ & $5: 22: 28.42$ & $1.8 \pm 0.5$ & $1.1 \pm 0.1$ & $0.23 \pm 0.01$ & $0.21 \pm 0.01$ & $65 \pm 10$ & $0.78_{-0.06}^{0.06}$ & 0.98 \\
\hline 8 & 4:57:07 & $6: 45: 29.63$ & $4.7 \pm 0.8$ & $3.7 \pm 0.2$ & $0.89 \pm 0.03$ & $0.93 \pm 0.03$ & $808 \pm 28$ & $0.48_{-0.06}^{0.06}$ & 5.34 \\
\hline 9 & 5:09:07 & $3: 48: 03.21$ & $1.9 \pm 0.6$ & $2.2 \pm 0.1$ & $0.34 \pm 0.01$ & $0.33 \pm 0.01$ & $114 \pm 10$ & $0.79_{-0.05}^{0.05}$ & 4.26 \\
\hline 10 & $5: 07: 35$ & $3: 08: 00.44$ & $4.1 \pm 0.8$ & $4.8 \pm 0.2$ & $1.13 \pm 0.03$ & $1.10 \pm 0.03$ & $634 \pm 23$ & $0.52_{-0.05}^{0.05}$ & 3.89 \\
\hline 11 & $5: 06: 37$ & $2: 37: 11.02$ & $1.6 \pm 0.5$ & $1.3 \pm 0.1$ & $0.22 \pm 0.01$ & $0.22 \pm 0.01$ & $62 \pm 9$ & $0.83_{-0.06}^{0.06}$ & 1.88 \\
\hline 16 & 4:49:08 & 1:52:58.99 & $3.7 \pm 0.6$ & $2.9 \pm 0.1$ & $0.40 \pm 0.01$ & $0.38 \pm 0.01$ & $96 \pm 10$ & $0.90_{-0.05}^{0.05}$ & 4.16 \\
\hline 17 & 4:44:18 & $1: 54: 45.61$ & $5.5 \pm 0.7$ & $3.7 \pm 0.2$ & $0.65 \pm 0.02$ & $0.61 \pm 0.02$ & $211 \pm 13$ & $0.79_{-0.05}^{0.05}$ & 0.83 \\
\hline 18 & 4:59:43 & $5: 20: 06.76$ & $1.7 \pm 0.6$ & $1.0 \pm 0.1$ & $0.25 \pm 0.01$ & $0.24 \pm 0.01$ & $104 \pm 10$ & $0.65_{-0.06}^{0.06}$ & 0.04 \\
\hline 19 & $5: 05: 22$ & $4: 59: 57.84$ & $1.9 \pm 0.6$ & $1.2 \pm 0.1$ & $0.99 \pm 0.03$ & $0.96 \pm 0.03$ & $1022 \pm 33$ & $0.20_{-0.00}^{0.01}$ & 7.56 \\
\hline 20 & $4: 44: 38$ & $5: 46: 32.00$ & $2.7 \pm 0.8$ & $2.5 \pm 0.2$ & $0.96 \pm 0.03$ & $0.94 \pm 0.03$ & $434 \pm 19$ & $0.48_{-0.05}^{0.05}$ & 1.34 \\
\hline 21 & $4: 55: 38$ & $2: 54: 08.06$ & $2.4 \pm 0.7$ & $2.3 \pm 0.1$ & $0.45 \pm 0.01$ & $0.44 \pm 0.01$ & $100 \pm 12$ & $0.83_{-0.05}^{0.05}$ & 6.62 \\
\hline 22 & $5: 15: 31$ & 9:04:03.94 & $2.8 \pm 0.6$ & $1.7 \pm 0.1$ & $0.26 \pm 0.01$ & $0.26 \pm 0.01$ & $104 \pm 12$ & $0.80_{-0.06}^{0.06}$ & 0.18 \\
\hline 23 & $5: 13: 51$ & $1: 56: 58.07$ & $6.1 \pm 0.8$ & $6.6 \pm 0.2$ & $0.49 \pm 0.02$ & $0.50 \pm 0.02$ & $170 \pm 14$ & $0.91_{-0.05}^{0.04}$ & 9.87 \\
\hline 24 & $5: 11: 34$ & $2: 44: 32.41$ & $2.1 \pm 0.7$ & $1.5 \pm 0.1$ & $0.39 \pm 0.01$ & $0.36 \pm 0.01$ & $195 \pm 13$ & $0.59_{-0.06}^{0.06}$ & 0.50 \\
\hline 25 & $4: 52: 48$ & 4:01:01.85 & $3.2 \pm 0.7$ & $2.4 \pm 0.1$ & $0.36 \pm 0.01$ & $0.35 \pm 0.01$ & $96 \pm 12$ & $0.87_{-0.06}^{0.06}$ & 1.98 \\
\hline
\end{tabular}

Col 1: Source name; Col 2 and 3: source coordinates; Col 4: flux density from the VLSSr image at 74 MHz; Col 5: flux density from the TGSS image at $150 \mathrm{MHz}$; Col 6: flux density from the NVSS image at $1.4 \mathrm{GHz}$; Col 7: flux density from the combined SRT+NVSS image at 1.4 GHz; Col 8: flux density from the GB6 image at 4.85 GHz; Col 9: spectral index between $74 \mathrm{MHz}$ and $1.4 \mathrm{GHz}$; Col 10: reduced chi-squared.

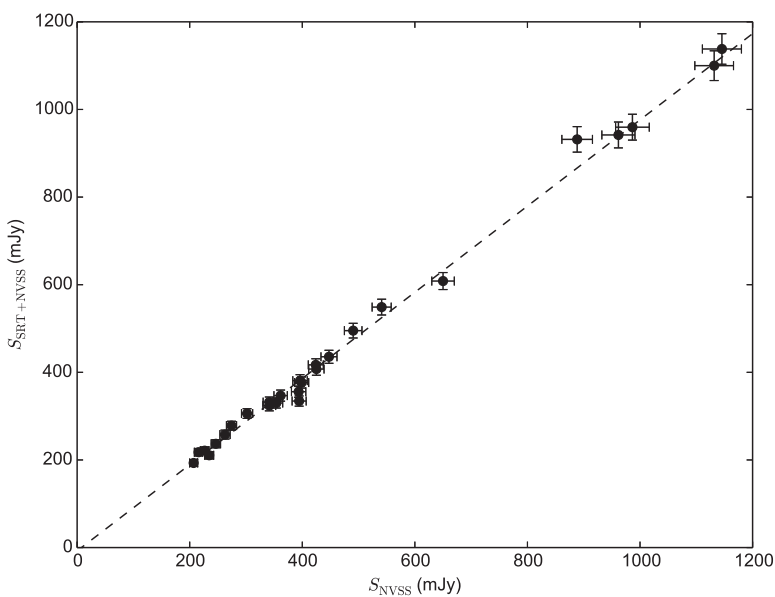

Figure A1. Flux from NVSS mosaic image versus flux from the combined SRT+NVSS image for sources with fluxes $>200 \mathrm{mJy}$.

10 percent if lower than this, to take into account any systematic errors. In Table A1, we report the spectral indices values, its error and the $\chi_{\text {red }}^{2}$ from the fit. All the sources have spectral index ranging from a minimum value of about 0.2 to a maximum value of about 1.1 , with a mean $\alpha=0.76$ and a standard deviation $\sigma_{\alpha}=0.19$. Plot of the flux densities versus frequency and the corresponding fits are shown in Figs A2 and A3 for each source. The VSLSSr clean bias has not been considered here, but we expect this correction to be negligible.

\section{APPENDIX B: OTHER INTERESTING SOURCES}

In Fig. A4, we show the SRT+NVSS contours overlaid on the SZ Y-map from the Planck satellite and the optical emission from the second Digitized Sky Survey (DSS2) red filter in the direction of two radio galaxies we serendipitously found in Region F (Fig. 13) and in Region H (Fig. 15), by inspecting the SRT+NVSS combined image. The source in the top panel of Fig. A4 is a candidate giant radio galaxy. It sits in the plane of the sky and is not present in the catalogues available in the literature. This radio source is located at RA (J2000) 05h:09m:50s and Dec. (J2000) +04 $: 20^{\prime}: 19^{\prime \prime}$. Its flux density at $1.4 \mathrm{GHz}$ is $(46 \pm 3) \mathrm{mJy}$ and its angular size is about 20 arcmin, as measured by the combined SRT+NVSS image. On the basis of its properties, we classify it as a Fanaroff-Riley (FR) II radio galaxy with two bright hotspots and two low-brightness jets. The nucleus of this radio galaxy is clearly detected, while the optical emission is below the noise level. Since radio galaxies are usually giant elliptical galaxies with absolute magnitude of at least -19 and the limiting apparent magnitude of the optical DSS2 

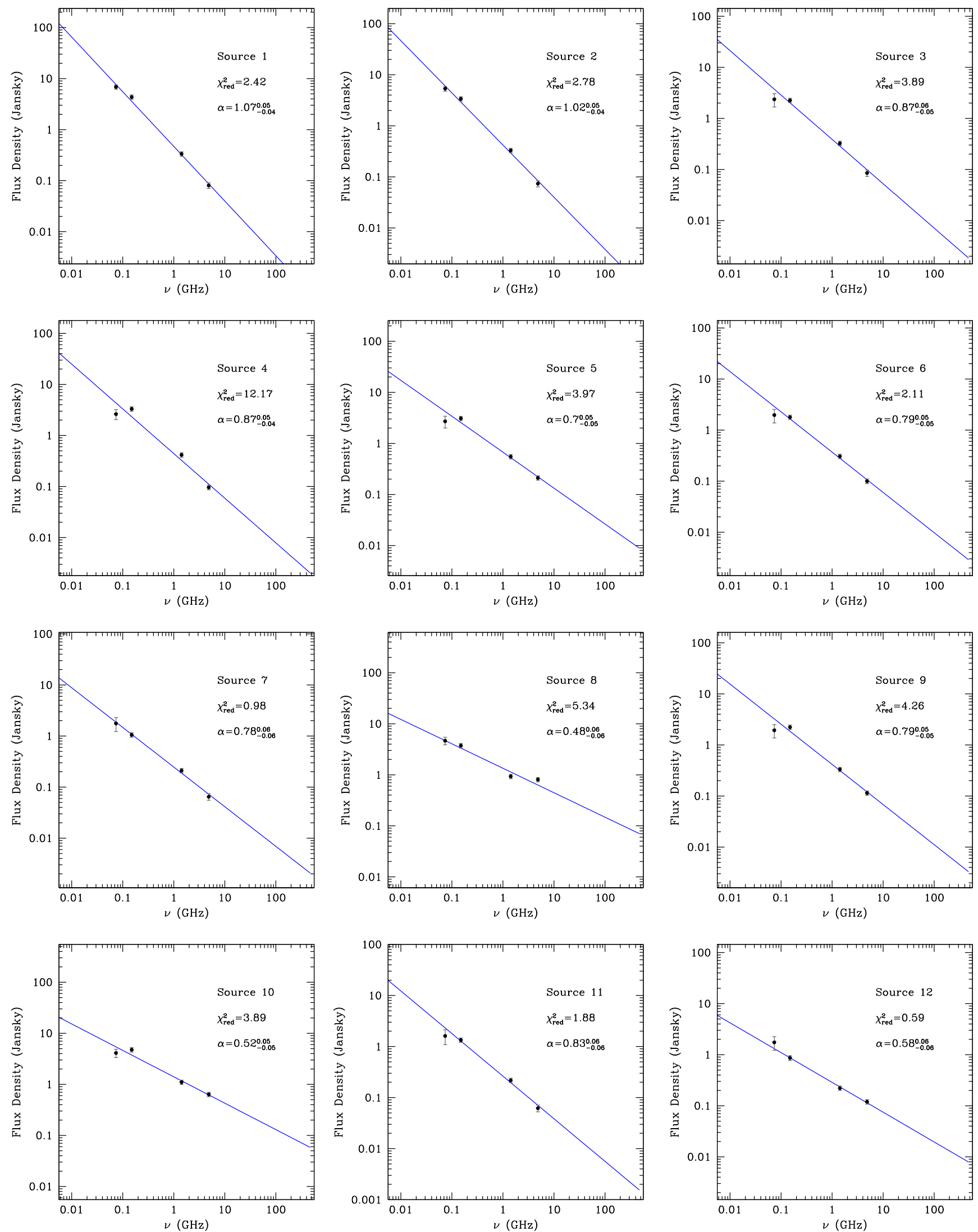

Figure A2. Flux density versus frequency for the first 12 sources in the catalogue. For each source the flux density as measured by VLSSr, TGSS, combined SRT+NVSS, and GB6 images is shown along with the power-law fit, the resulting spectral index $\alpha$ value, and the $\chi_{\text {red }}^{2}$ of the fit. 

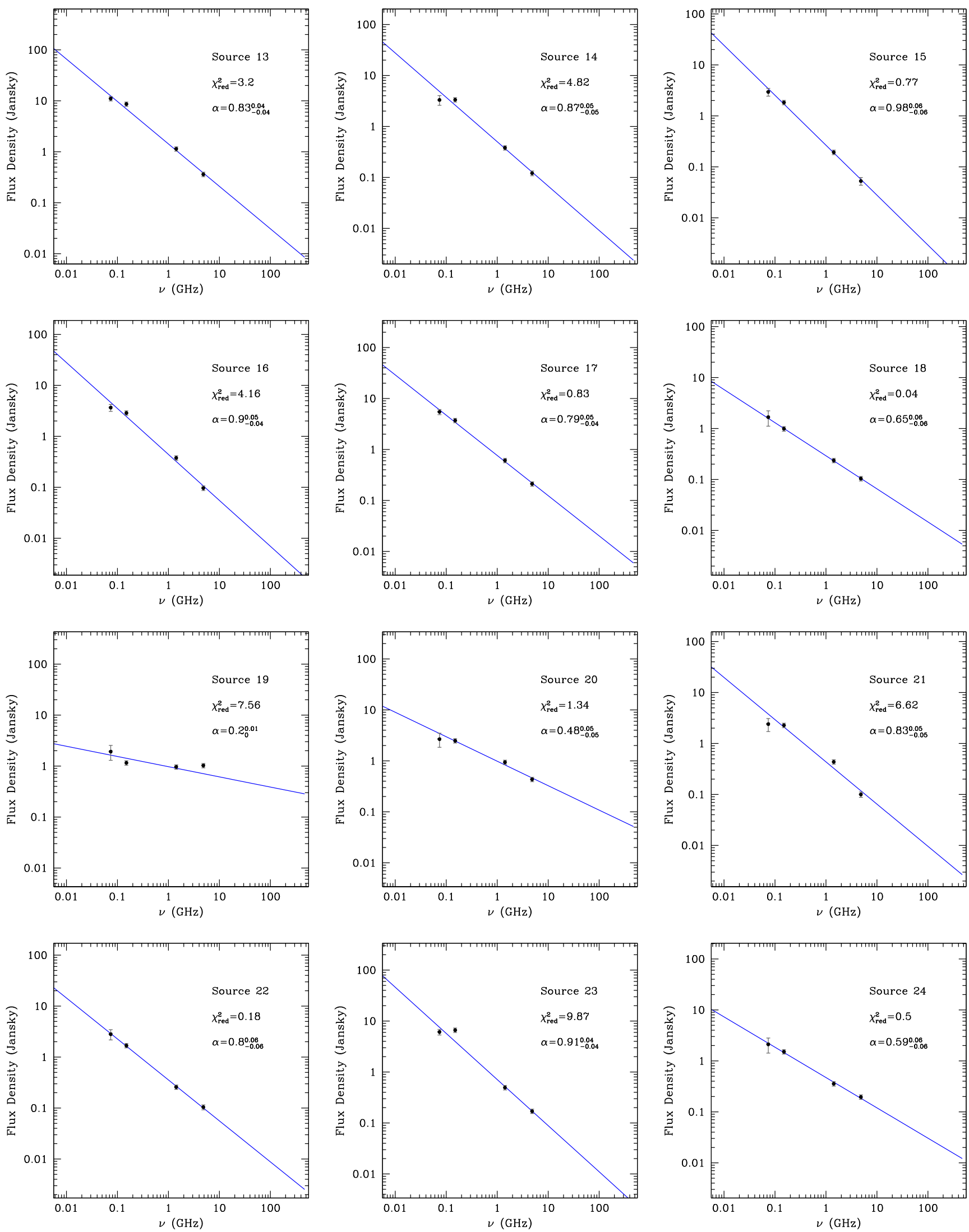

Figure A3. Flux density versus frequency for the last 12 sources in the catalogue. For each source the flux density as measured by VLSSr, TGSS, combined SRT+NVSS, and GB6 images is shown along with the power-law fit, the resulting spectral index $\alpha$ value, and the $\chi_{\text {red }}^{2}$ of the fit. 

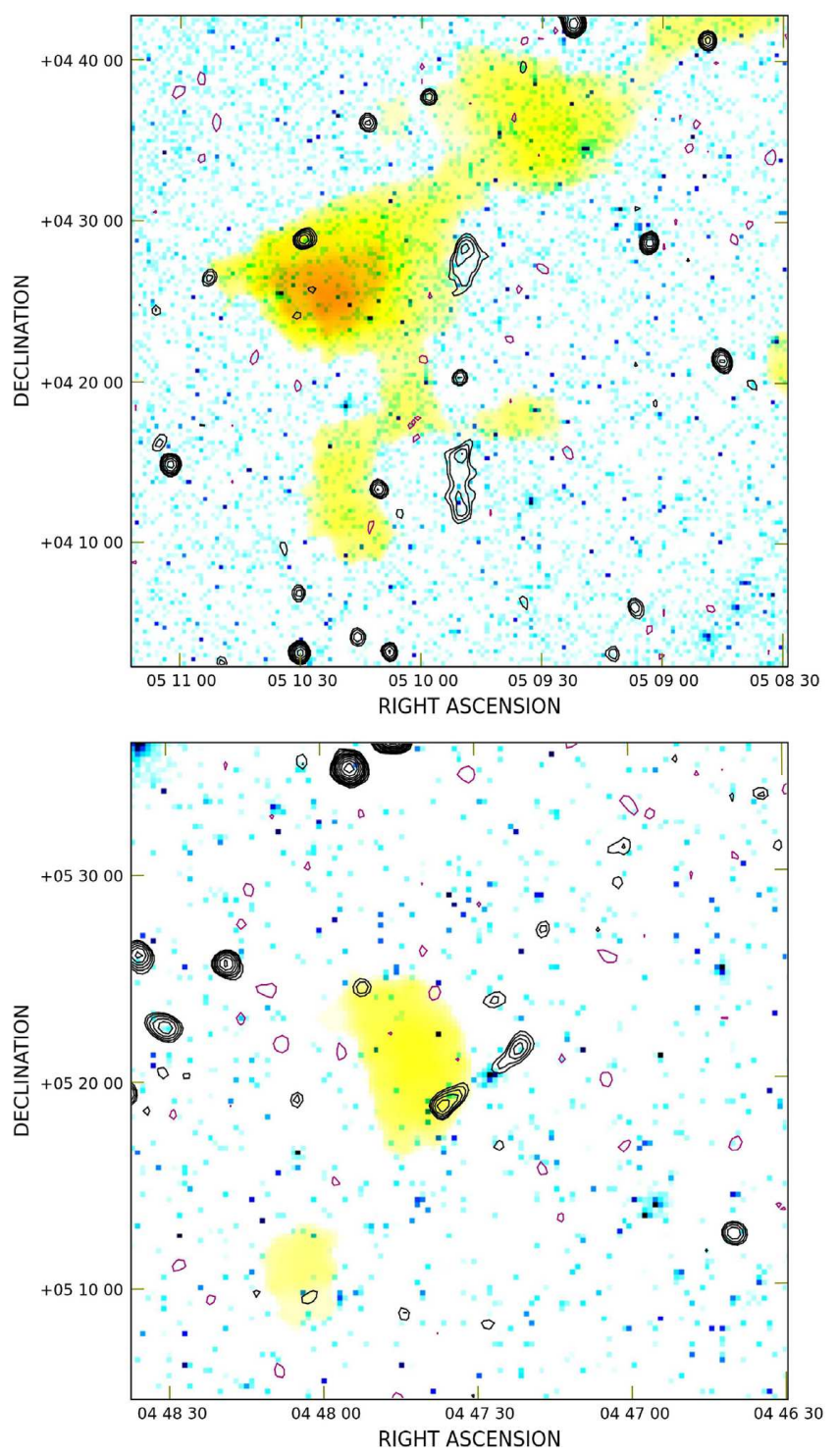

Figure A4. SRT+NVSS contours overlaid on the Planck Y-map and the optical DSS2 red image in colours (respectively yellow and blue), for the two radio galaxies (top from Region $\mathrm{F}$ and bottom from Region $\mathrm{H}$ ) described in Appendix B. The contour levels are $-1.35,1.35 \mathrm{mJy} \mathrm{beam}^{-1}$ (with a beam of $45 \mathrm{arcsec}$ ) and the remaining increase by a factor $\sqrt{2}$ (negative in magenta and positive in black).

red filter is 20.8, we derive that the source distance is larger than $0.9 \mathrm{Gpc}$. Considering that the angular size has a minimum for $z=1$, we infer that the size of this source ranges between 4 and $10 \mathrm{Mpc}$. Interestingly, the SZ Y-map reveals hints of cavities at the location of the lobes of the source.

The source in the bottom panel of Fig. A4 is another interesting source, located at RA (J2000) 04h:47m:23.9s Dec. (J2000) $+05 \mathrm{~d}: 18 \mathrm{~m}: 50 \mathrm{~s}$. It has a flux density at $1.4 \mathrm{GHz}$ of $(19 \pm 1) \mathrm{mJy}$ and its angular size is about 6 arcmin, as measured by the combined SRT+NVSS image. The closest systems with optical identification have a redshift $z \approx 0.1$. At this distance, the source would have a linear size of about $700 \mathrm{kpc}$. We classify it as an FRII radio galaxy.

\section{APPENDIX C: EFFECTS OF GAIN FLUCTUATIONS AND GALACTIC FOREGROUND}

Gain fluctuations of the receiving chain can affect the observed radio emission. To exclude that the new candidate sources are spurious patches of radio emission due to these gain fluctuations, we produced two $8^{\circ} \times 8^{\circ}$ RA-Dec. mock SRT observations of the sky and run them through the same imaging pipeline as the observations. To reduce the computational burden, the simulations were run only in the same frequency ranges as the NVSS (1364.9-1414.9 and 1435.1-1485.1 MHz) and then averaged.

As a first step, we characterize the noise properties of our images. For each $8^{\circ} \times 8^{\circ}$ RA-Dec. observation and each polarization, we extracted five streams of data points along the full sub-scan in cold regions of the sky. The total number of data points for each stream is about 850 and they have been acquired every $100 \mathrm{~ms}$ corresponding to a sub-scan duration of about $85 \mathrm{~s}$. For each data stream we derived the power spectrum of the noise fluctuations in the frequency range $0.01-5 \mathrm{~Hz}$ and all the power spectra were averaged, see Fig. C1. We interpreted the average observed noise spectrum as a combination of white noise with power spectrum $P_{\text {white }} \simeq 1\left(\mathrm{Jy} \mathrm{beam}^{-1}\right)^{2} \mathrm{~Hz}^{-2}$ corresponding, according to the radiometer equation, to a system temperature of $33 \mathrm{~K}$, for an integration time of $100 \mathrm{~ms}$, and a bandwidth of $50 \mathrm{MHz}$, and a $1 / f$-noise with power

$P_{1 / f}=P_{0}\left(f / f_{0}\right)^{-s}$,

where $P_{0}=3\left(\mathrm{Jy} \mathrm{beam}^{-1}\right)^{2} \mathrm{~Hz}^{-2}, f_{0}=1 \mathrm{~Hz}$, and $s=1.8$. In Fig. C1, we show the observed power spectrum of the noise (blue), the whitenoise (horizontal red dashed line) component, the $f$-noise (diagonal red dashed line) component, and the sum of the white and $1 / f$-noise (red continuous line).

To investigate whether these gain fluctuations can produce spurious large-scale radio emission, we produced mock observations with the same noise properties as the observations and a point-like source sky model. We assumed a system temperature $T_{\text {sys }}=33 \mathrm{~K}$, a gain $G=0.47 \mathrm{~K} \mathrm{Jy}^{-1}$, a forward efficiency $\eta_{\mathrm{F}}=1$, and a flat gain curve (see Bolli et al. 2015). We adopted a ground temperature $T_{\text {ground }}=303 \mathrm{~K}$ and a zenith opacity $\tau=0.006$, as

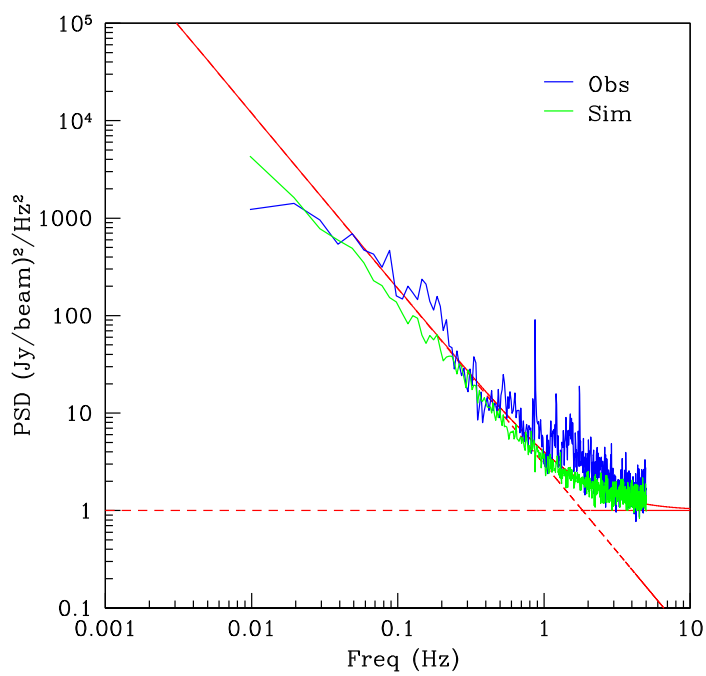

Figure C1. Power spectrum of the noise derived from the observations (blue) and from the simulations (green). The horizontal and diagonal red dashed lines represent respectively the white-noise and $1 / f$-noise components, while the red continuous line is the sum of white and $1 / f$-noise. 

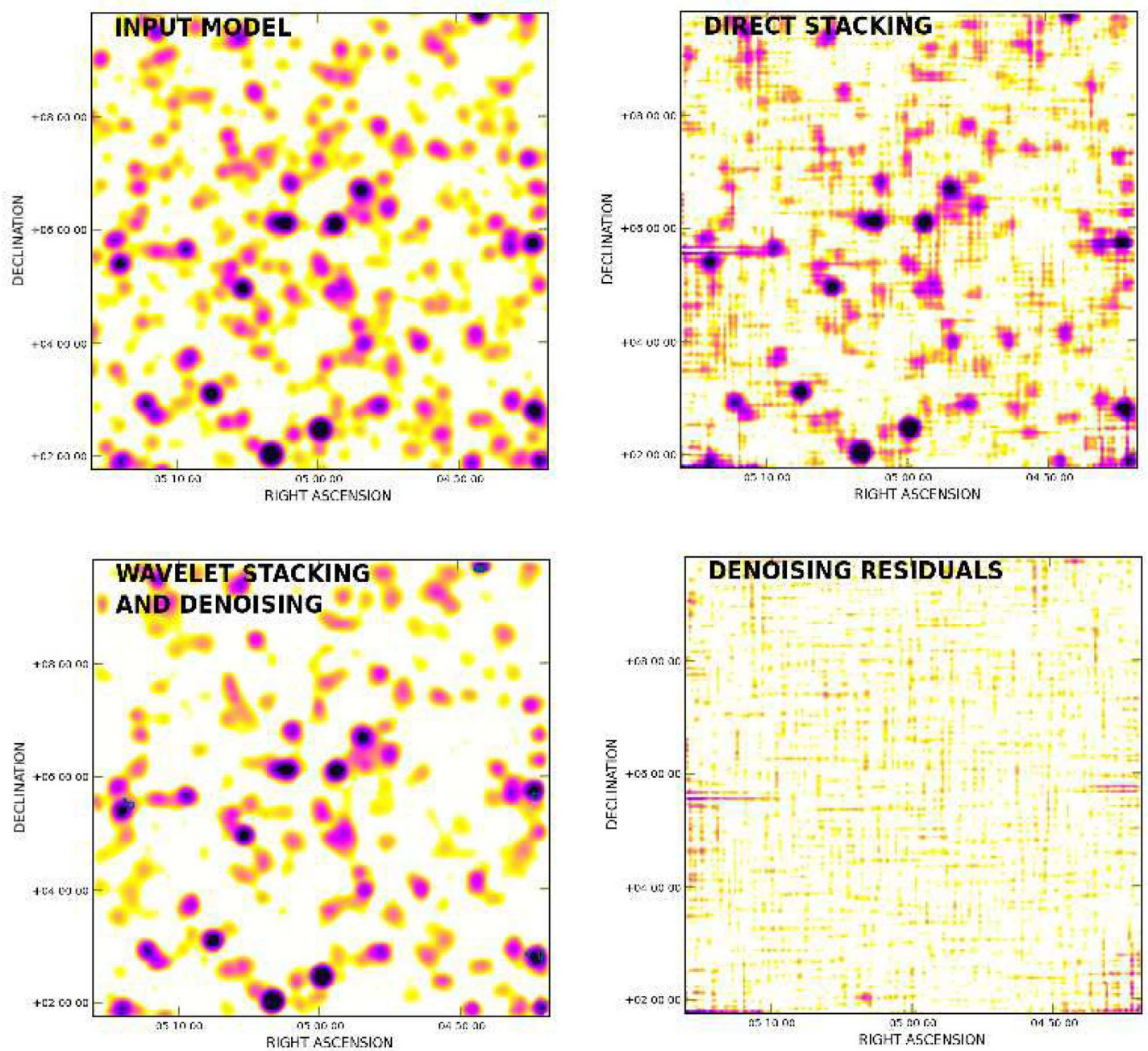

Figure C2. Top left panel: Model of the sky including only the point-like sources. Top right panel: The image derived by direct averaging all the RA and Dec. baseline subtracted scans. Bottom left panel: Image after applying the wavelet stacking and denoising algorithms. The blue contours represent the difference between the wavelet stacking and denoising image and the input model and start from $3 \sigma$ of the first. Bottom right panel: Difference of the last two panels.
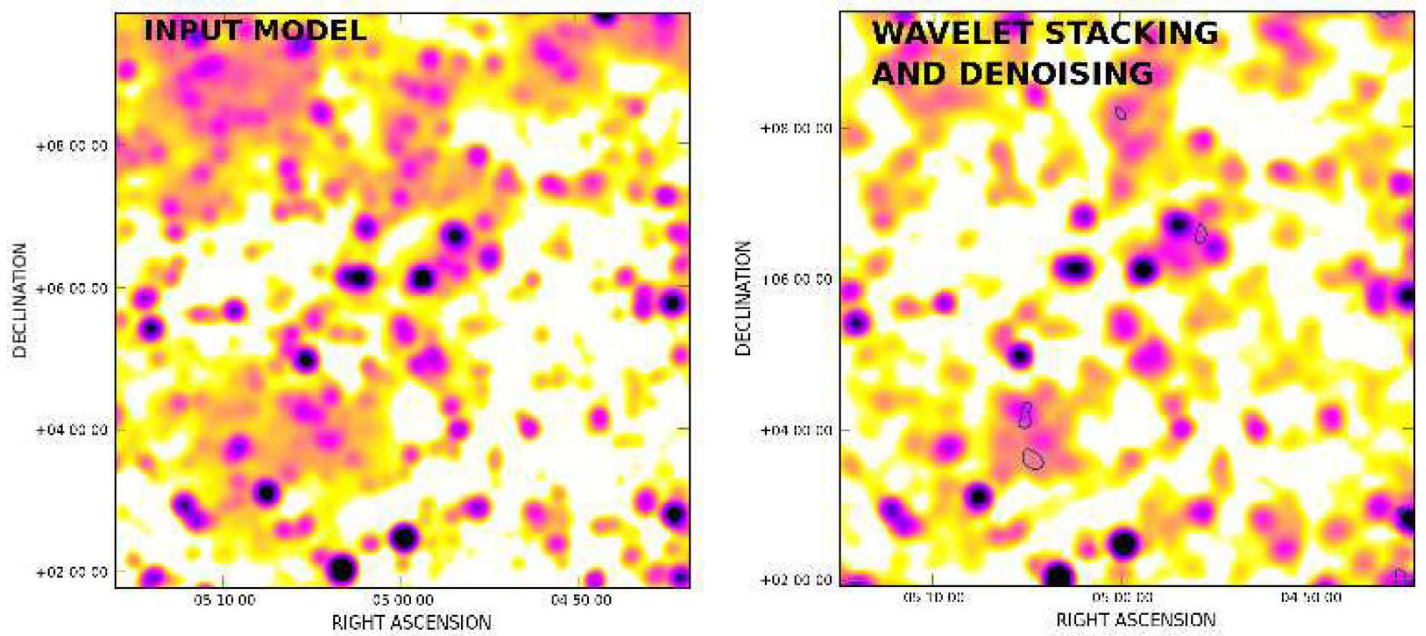

Figure C3. Left-hand panel: Model of the sky including the point-like sources and a model of our Galaxy foreground. Right-hand panel: The colours represent the radio emission obtained by applying the imaging pipeline to mock observations including a Galactic and a point-like source contribution. Blue contours show the diffuse synchrotron patches after the NVSS point-like source model has been subtracted and start from $3 \sigma$ of the wavelet stacking and denoising image. 
registered at the time of our observations. The sky model has been obtained from the NVSS by applying a cut in brightness of $10 \sigma_{\mathrm{NVSS}}=4.5 \mathrm{mJy}$ beam $^{-1}$. The image has been convolved to the SRT resolution at the NVSS frequency (13.66 arcmin). In Fig. C1, we show for consistency the averaged power spectrum of the noise derived from the mock data in green. At high frequencies, the observed power spectral density is slightly higher than the mock one. This is probably due to the fact that the mock data do not include the confusion noise, naturally present in the observations.

In Fig. C2, we compare the model to the images obtained after the pipeline: the model of the sky is shown in the top left panel, the image derived by direct averaging all the RA and Dec. baseline subtracted scans in the top right panel, the image after mixing and denoising algorithms in the bottom left panel, and the difference of the last two in the bottom right panel. The top and bottom right panels show that gain fluctuations are present along the sub-scan direction (with autocorrelation length of about $10 \mathrm{~s}$ ) but appear to be completely de-correlated between a sub-scan and those immediately before and after. After mixing and denoising, these small-scale fluctuations are strongly reduced. This analysis indicates that the new candidate sources are not the result of gain fluctuations of the receiving system.

To investigate whether these sources could be of Galactic origin, we repeated the same procedure after including the Galactic contribution in the model. We produced the mock Galactic emission by using a power spectrum derived from the Stockert survey (Reich 1982) around the field of interest. The Stockert survey has a resolution of 35 arcmin, coarser than the SRT. The power spectrum of the Galactic foreground estimated from the Stockert survey has been extrapolated down to the cell-size of the Stockert image (15 arcmin, comparable to the SRT resolution). Moreover, it should be noted that most of the power is on large scales. The mock Galactic emission was added to the NVSS point-like source model described before. In Fig. C3, we show on the left the model and on the right the image after using the same pipeline as for the observations in colours, while in contours the diffuse synchrotron patches after the NVSS point-like source model has been subtracted. Apart from structures at the edges of the image, five patches survive in the final image. Therefore, we conclude that only about $18-20$ percent of the new candidate sources we discover could be of Galactic origin.

${ }^{1}$ INAF - Osservatorio Astronomico di Cagliari, Via della Scienza 5, I-09047 Selargius (CA), Italy

${ }^{2}$ Dipartimento di Fisica, University of Cagliari, Strada Prov.le MonserratoSestu Km 0.700, I-09042 Monserrato (CA), Italy
${ }^{3}$ Dipartimento di Fisica e Astronomia, Università degli Studi di Bologna, Viale Berti Pichat 6/2, I-40127 Bologna, Italy

${ }^{4}$ INAF - Istituto di Radioastronomia, Via Gobetti 101, I-40129 Bologna, Italy

${ }^{5}$ Hamburger Sternwarte, Universität Hamburg, Gojenbergsweg 112, D21029 Hamburg, Germany

${ }^{6}$ Department of Physics, University of Helsinki, Gustaf Hällströpmin katu 2a, FI-00014 Helsinki, Finland

${ }^{7}$ CSCS-ETHZ, Via Trevano 131, 6900 Lugano, Switzerland

${ }^{8}$ Agenzia Spaziale Italiana (ASI), 00133 Roma, Italy

${ }^{9}$ SKA SA, 3rd Floor, The Park, Park Road, Pinelands 7405, The Cape Town, South Africa

${ }^{10}$ Department of Physics and Electronics, Rhodes University, PO Box 94, Grahamstown 6140, South Africa

${ }^{11}$ Fundación G. Galilei - INAF TNG, Rambla J. A. Fernández Pérez 7, E-38712 Breña Baja (La Palma), Spain

${ }^{12}$ Instituto de Astrofísica de Canarias, C/Vía Láctea s/n, E-38205 La Laguna (Tenerife), Spain

${ }^{13}$ Departamento de Astrofísica, Universidad de La Laguna, Av. del Astrofísico Francisco Sánchez s/n, E-38205 La Laguna (Tenerife), Spain

${ }^{14}$ ASTRON, the Netherlands Institute for Radio Astronomy, Postbus 2, NL7990 AA Dwingeloo, the Netherlands

${ }^{15}$ Naval Research Laboratory, Washington, DC 20375, USA

${ }^{16}$ School of Physics, University of the Witwatersrand, Private Bag 3, 2050 Johannesburg, South Africa

${ }^{17}$ Max Planck Institut für Astrophysik, Karl-Schwarzschild-Str 1, D-85740 Garching, Germany

${ }^{18}$ Laboratoire Lagrange, UCA, OCA, CNRS, Blvd de l'Observatoire, CS 34229, F-06304 Nice cedex 4, France

${ }^{19}$ University of Leiden, Rapenburg 70, NL-2311 EZ Leiden, the Netherlands

${ }^{20}$ INAF - IASF Milano, Via Bassini 15, I-20133 Milano, Italy

${ }^{21}$ Department of Physics and Astronomy, University of California at Irvine, 4129 Frederick Reines Hall, Irvine, CA 92697-4575, USA

${ }^{22}$ Dipartimento di Fisica, dell'Università degli Studi di Trieste - Sezione di Astronomia, via Tiepolo 11, I-34143 Trieste, Italy

${ }^{23}$ INAF - Osservatorio Astronomico di Trieste, via Tiepolo 11, I-34143 Trieste, Italy

${ }^{24}$ International Centre for Radio Astronomy Research, Curtin University, Bentley, WA 6102, Australia

${ }^{25}$ Argelander-Institut für Astronomie, Auf dem Hügel 71, D-53121 Bonn, Germany

${ }^{26}$ National Radio Astronomy Observatory, PO Box O, Socorro, NM 87801 , USA

${ }^{27}$ Department of Physics and Astronomy, University of New Mexico, Albuquerque, NM 87131, USA

This paper has been typeset from a $\mathrm{T}_{\mathrm{E}} \mathrm{X} / \mathrm{L} \mathrm{T}_{\mathrm{E}} \mathrm{X}$ file prepared by the author. 